\title{
Analysis of small scale solar magnetic fields using Hinode SOT/SP
}

\author{
Dissertation \\ for the award of the degree \\ “Doctor rerum naturalium” (Dr.rer.nat.) \\ of the Georg-August-Universität Göttingen \\ within the doctoral program PROPHYS \\ of the Georg-August University School of Science (GAUSS)
}

\author{
submitted by \\ David Bühler \\ from Itzehoe
}

Göttingen, 2013 


\section{Thesis Committee}

Prof. Dr. Sami K. Solanki

Max-Planck-Institut für Sonnensystemforschung, Katlenburg-Lindau, Germany

Prof. Dr. Stefan Dreizler

Institut für Astrophysik, Georg-August-Universität Göttingen, Germany

Dr. Andreas Lagg

Max-Planck-Institut für Sonnensystemforschung, Katlenburg-Lindau, Germany

Members of the Examination Board

Reviewer: Prof. Dr. Stefan Dreizler

Institut für Astrophysik, Georg-August-Universität Göttingen, Germany

Second Reviewer: Prof. Dr. Sami K. Solanki

Max-Planck-Institut für Sonnensystemforschung, Katlenburg-Lindau, Germany

Additional Reviewer: Prof. Dr. Wolfgang Schmidt

Kiepenheuer-Institut für Sonnenphysik, Albert-Ludwigs-Universität Freiburg, Germany

Further members of the Examination Board:

Prof. Dr. Manfred Schüssler

Max-Planck-Institut für Sonnensystemforschung, Katlenburg-Lindau, Germany

Prof. Dr. Laurent Gizon

Institut für Astrophysik, Georg-August-Universität Göttingen, Germany

Prof. Dr. Wolfgang Glatzel

Institut für Astrophysik, Georg-August-Universität Göttingen, Germany

Prof. Dr. Hardi Peter

Max-Planck-Institut für Sonnensystemforschung, Katlenburg-Lindau, Germany

Date of the oral examination: 7. November 2013 


\section{Bibliografische Information der Deutschen Nationalbibliothek}

Die Deutsche Nationalbibliothek verzeichnet diese Publikation in der Deutschen Nationalbibliografie; detaillierte bibliografische Daten sind im Internet über http: //dnb . d-nb. de abrufbar.

ISBN 978-3-942171-78-6

uni-edition $\mathrm{GmbH} 2014$

http://www.uni-edition.de

(C) David Bühler

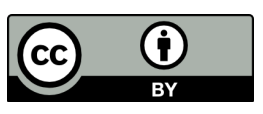

This work is distributed under a

Creative Commons Attribution 3.0 License

Printed in Germany 



\section{Contents}

\begin{tabular}{ll}
\hline Summary & 7
\end{tabular}

\begin{tabular}{lll}
\hline 1 & Introduction & 9
\end{tabular}

1.1 Zeeman effect . . . . . . . . . . . . . . . . . . . . . 11

1.2 Hinode SOT/SP $\ldots \ldots \ldots \ldots$. . . . . . . . . . . . . . . 13

1.3 Inversions $\ldots \ldots \ldots \ldots \ldots \ldots \ldots$

1.4 Internetwork magnetic fields $\ldots \ldots \ldots \ldots$. . . . . . . . . 15

1.4 .1 Observations . . . . . . . . . . . . . . . . 15

1.4 .2 Origin . . . . . . . . . . . . . . . . . 17

1.5 Magnetic flux tubes $\ldots \ldots \ldots \ldots$

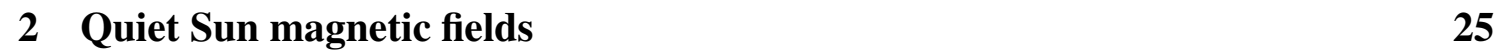

2.1 Abstract . . . . . . . . . . . . . . . . . . 25

2.2 Introduction . . . . . . . . . . . . . . . . . . 25

2.3 Data and method of analysis . . . . . . . . . . . . . . . 26

2.3 .1 Instrumental effects . . . . . . . . . . . . . . . . 27

2.3 .2 Image analysis . . . . . . . . . . . . . . . . 28

2.4 Results: circular polarisation . . . . . . . . . . . . . . . . . . . 30

2.5 Results: linear polarisation . . . . . . . . . . . . . . . . . . . . . . . . . . . . . . 34

2.6 Discussion and conclusion $\ldots \ldots \ldots \ldots$. . . . . . . . . . . . 37

3 Properties of solar plage 43

3.1 Abstract . . . . . . . . . . . . . . . . . . . 43

3.2 Introduction . . . . . . . . . . . . . . . . . . . . . . . 43

3.3 Data . . . . . . . . . . . . . . . . . . 45

3.4 Inversions . . . . . . . . . . . . . . . . . . . 46

3.5 Results . . . . . . . . . . . . . . . . . . . . . 47

3.5 .1 Magnetic field strength . . . . . . . . . . . . . . . 50

3.5 .2 Velocities . . . . . . . . . . . . . . . . . . . . . . . . . . . 53

3.5 .3 Temperature . . . . . . . . . . . . . . . . 56

3.5 .4 Inclination \& Azimuth . . . . . . . . . . . . . . . . 60

3.5 .5 Magnetic field gradient . . . . . . . . . . . . . . . . 63

3.5.6 Expansion of magnetic features with height . . . . . . . . . . . . 64

3.5.7 Effect of the sunspot $\ldots \ldots \ldots \ldots 6$

3.5 .8 Microturbulence . . . . . . . . . . . . . . . . . 67

3.6 Discussion and Conclusion $\ldots \ldots \ldots$. . . . . . . . . . . . . . . 69 
4 Analysis of twist in solar plage $\quad \mathbf{7 3}$

4.1 Introduction . . . . . . . . . . . . . . . . . . . . 73

4.2 Data and Inversions . . . . . . . . . . . . . . . . . . 74

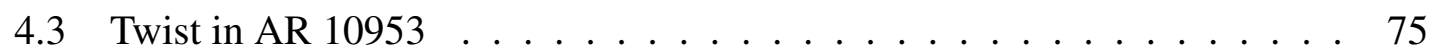

4.4 Twist in ARs across the solar disc . . . . . . . . . . . . . . . 81

4.5 Cross talk . . . . . . . . . . . . . . . . . . . . 81

4.6 Absorption line damping . . . . . . . . . . . . . . . . . . 86

4.7 Other tests . . . . . . . . . . . . . . . . . . . . . . . . . 88

4.8 Conclusion $\ldots \ldots \ldots \ldots$

\begin{tabular}{lll}
\hline & Conclusion \& Outlook & 91
\end{tabular}

\begin{tabular}{ll}
\hline Bibliography & 95
\end{tabular}

\begin{tabular}{ll}
\hline Acknowledgements & 113
\end{tabular}

\begin{tabular}{ll}
\hline Scientific contributions & 115
\end{tabular}

\begin{tabular}{ll}
\hline Lebenslauf & 117
\end{tabular} 


\section{Summary}

This thesis presents an analysis of small magnetic structures found in the solar photosphere. All the work presented in the following chapters is primarily based on data obtained from the spectropolarimeter aboard the Hinode satellite. The spectropolarimeter provides seeing-free, high spectral resolution $(30 \mathrm{~m} \AA)$ and spatial (0."32) resolution data of the full Stokes vector of the Zeeman sensitive Fe I line pair at $6300 \AA$.

Chapter 2 investigates internetwork magnetic fields found on the disc centre of the quiet Sun. These fields are characterized by their predominantly sub- $k G$ strength, their short lifetimes on the order of minutes and the fact that they encompass most of the unsigned magnetic flux on the solar surface, at any time. Many properties of these magnetic fields are still unclear, however, one of which is their origin. It has been, both, proposed and separately demonstrated using magneto-hydrodynamic simulations, that such fields may be produced by local surface dynamo action, which acts independently from the global, active-region producing solar dynamo. Should the majority of the internetwork magnetic fields be produced by a local dynamo then no solar cycle dependence should be seen in them. With the stable and seeing-free data offered by Hinode we have been able to monitor internetwork magnetic fields from November 2006 until May 2012, which is approximately equivalent to half a solar cycle. After taking into account instrumental effects influencing the measurement of the Stokes vector over time, we found no significant variation in, both, horizontally and vertically orientated magnetic fields found in the internetwork. This result supports the local dynamo scenario as the principal source of these fields.

Chapters $3 \& 4$ analyse magnetic fields in plage, which are typically found surrounding sunspots in active regions. Plage is identified easily by intensity enhancements in temperature sensitive spectral lines, or as an accumulation of bright points in the continuum and is composed of $k G$ magnetic fields in the form of thin flux tubes or sheets. Even though the analysis of magnetic fields in plage has an expansive and thorough literature and history, the typical size of flux tubes in the photosphere of only a few $100 \mathrm{~km}$ challenges even current solar telescopes and, therefore, many question regarding their properties are left unanswered. In this investigation we inverted the Hinode observations using the SPINOR code, thereby reconstructing the solar atmosphere at the time of observation, whilst taking into account the point-spread-function of the telescope and allowing for line-of-sight gradients of the various atmospheric parameters. The inversion was able to confirm many of the previously known properties of these magnetic fields, such as a typical field strength of $1.5 k G$, typical inclinations of $10^{\circ}-15^{\circ}$ with respect to the surface, and an absence of strong stationary plasma flows, as well as a temperature enhancement within the magnetised region when compared to the quiet Sun in particular in the upper photosphere. In addition, we were able to directly observe the expansion of magnetic features with height and found it to be compatible with a thin flux tube model. The inversion results allowed 
us to clearly distinguish between fields forming part of a magnetic canopy from those forming the root or core of a feature. Furthermore, we observed that each magnetic feature was surrounded by a ring of strong downflows, typically with speeds of $1-3 \mathrm{~km} / \mathrm{s}$, but velocities of up to $8 \mathrm{~km} / \mathrm{s}$ were observed in isolated cases. Within these strong downflows we noticed small magnetic patches with pixel averaged field strengths of $100 G$ bearing the opposite polarity of the main magnetic feature, which were effectively hidden beneath stronger canopy fields. The ring of downflows agree well with simulations of the solar photosphere and, in particular, the weak magnetic fields found within them had eluded observational detection so far. Each magnetic feature was also enveloped by a ring of enhanced microturbulent velocities when compared to the quiet Sun, which could hint at unresolved velocity structures or waves at the interface between the magnetic feature and the quiet Sun. The azimuthal orientation of magnetic fields in plage is in general isotropic except when found in proximity to another large magnetic structure, such as a sunspot. Furthermore, the azimuthal orientation of the magnetic fields was found to be height dependent, implying a twist. As part of a follow up investigation several plage regions of different active regions were analysed and a twist was revealed to be present in every active region that was considered. However, the twist failed to show a hemispherical dependence as predicted by Hale's polarity law. Several instrumental effects, such as cross talk between Stokes parameters and uncertainties in the absorption line damping constants employed by the inversion, were tested to ascertain a possible instrumental or inversion-based error as an explanation of the observed twist. So far such a source, which conclusively explains the observed twists has not been found. 


\section{Introduction}

The Sun, a G2V main sequence star, would be rather unremarkable were it not to host magnetic fields, which give it considerable dynamism and complexity. A complete introduction to the Sun's magnetic field and its numerous effects and manifestations would not only put unnecessary strain on a reader's attention span but, more importantly, would by far exceed the scope of this introduction. Hence, right from the outset it must be said that the focus of this thesis will be firmly rooted in the solar photosphere, a narrow layer in the solar atmosphere a few hundred kilometers in height, where the solar plasma turns from being opaque to most radiation at visible wavelengths to being highly transparent. The processes taking place in the interior of the Sun, giving rise to the Sun's magnetic fields, as well as the atmospheric layers above the photosphere, namely the Chromosphere, Transition region and Corona, will be discussed only in passing and from a photospheric point of view and may, therefore, not receive the attention they would otherwise deserve. The photosphere and its magnetic fields, nonetheless, provide numerous and interesting challenges that often defy straight forward interpretations. Solar observatories, be it space-, balloon-, or ground-based, have been instrumental in characterizing the basic structure and processes in the photosphere and modern solar observatories have only begun to resolve many of its finer details. This thesis will build upon and extend that effort, by using photospheric observations from the space-borne Hinode satellite in an attempt to uncover some properties of small scale magnetic fields in the photosphere.

After a brief overview of the photosphere, a more detailed introduction will be given to both the internetwork magnetic fields and magnetic flux tubes.

The Sun, like many G, K and M spectral type stars, possesses a magnetic field that changes markedly with time, of which the 11 year sunspot cycle is one of its most readily identifiable features (Schwabe 1844). The smoothed sunspot number plotted in Fig. 1.1 displays that in general successive cycles are far from identical and already hints that beyond the 11 year sunspot cycle the Sun's magnetic activity may vary on even longer time scales. The $17^{\text {th }}$ century Maunder minimum (Eddy 1976), a time during which no sunspots were recorded, not only demonstrates the great temporal variability of the Sun's magnetic field but also becomes nothing but an episode in comparison to the ever changing alternations between the Sun's grand activity maxima and minima when even longer time scales are considered (Solanki et al.2004). On shorter time scales the Sun also displays considerable activity. During the activity maximum of a solar cycle new sunspots can emerge and form in a matter of days (Solanki 2003), whilst some of the smallest magnetic features can emerge and fragment within mere minutes (Ishikawa et al. 2008).

The solar magnetic fields show great variability on spatial scales as well. In the photosphere sunspots with typical sizes ranging from 30" to 40" (Borrero and Ichimoto 2011) are the 


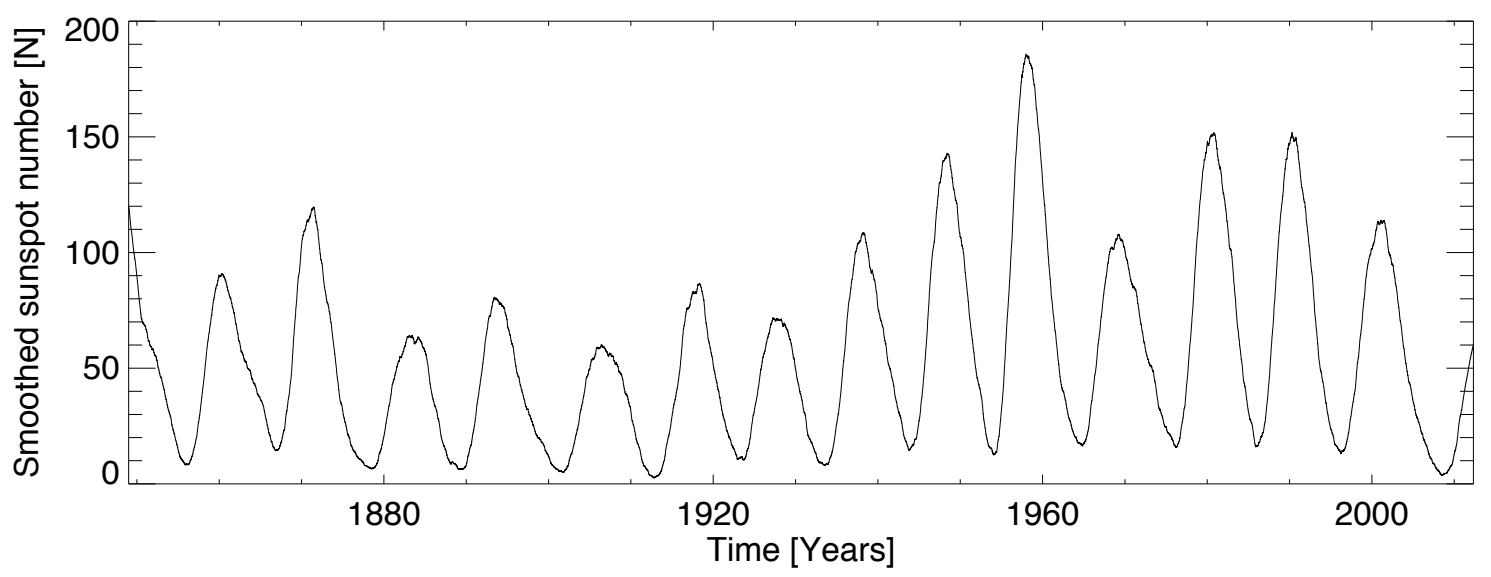

Figure 1.1: Variation of the yearly smoothed International sunspot number record with time.

largest identifiable features on the solar disc, whose magnetic nature was determined for the first time by Hale (1908) using the Zeeman effect. With the advent of magnetograph whereby the polarized light of the $\sigma$ and $\pi$ components of a Zeeman sensitive absorption line is analysed, Babcock and Babcock (1955) showed that magnetic fields are also prevalent on the solar surface outside sunspots. Many smaller magnetic features found in active regions (ARs) typically arrange themselves in a ribbon-like manner (Berger et al. 2004). They are commonly associated with intensity brightenings known as faculae or plage observed in spectral lines like Ca II (Solanki 1993). Outside ARs, often called the quiet Sun, magnetic fields are found at the edges of adjoining supergranulation cells (Leighton et al. 1962; Simon and Leighton 1964) to form what is referred to as the network. Supergranulation cells are found over the entire solar surface and possess a typical diameter of 20" to 40" (Rieutord and Rincon 2010). Within the supergranulation cells some of the most intermittent and smallest magnetic features, often less than one arcsecond in diameter can be found (de Wijn et al.2009). Magnetic fields found in these regions are commonly called internetwork magnetic fields.

In the photosphere the magnetic fields are structured by convection patterns. The granular convection pattern is the most easily observed and consists of hot, bright granules of rising plasma on average one arcsecond in diameter, which are separated by narrow intergranular lanes of cool, sinking plasma. The high magnetic Reynolds number in the photosphere causes any magnetic field to be frozen-in-flux and to be carried along by the convective flows. The granulation pattern is continuously changing due to the short granular life time of five to ten minutes (de Wijn et al.2009). The aforementioned supergranulation pattern presents a flow field superimposed upon the granular flows, which slowly advects magnetic elements to supergranular boundaries. A typical supergranule has a life time of 20 hours (Rieutord and Rincon 2010). A third intermediate convection scale called mesogranulation having a typical scale of 5 to 10 arcsconds is often reported, although its existence is more disputed than either granulation or supergranulation (Roudier et al.|1999).

The transportation of magnetic fields by convective flows in the photosphere inevitably causes them to be concentrated in the downflow regions such as the intergranular lanes, a process called flux expulsion (Parker 1963). The convection flow can concentrate magnetic 
fields up to the equipartition field strength, which in the photosphere is $400 \mathrm{G}$ on average. However, following the analysis by Stenflo (1973) 90\% of the magnetic fields on the solar surface were shown to be concentrated in small $k G$ structures in the form of flux tubes or sheets, at resolution of one arcsecond or more and comparatively low polarimetric sensitivity. The convective collapse mechanism, thought to drive the intensification of the magnetic fields beyond $h G$ values, occurs when the plasma within a $h G$ magnetic field concentration cools radiatively and sinks, whilst, due to the Lorentz force, the lateral inflow of hot plasma is reduced. In response the magnetic field starts to contract until a new pressure balance between its interior and exterior is reached (Parker 1976). The resulting flux tube/sheet typically has a diameter of only 100km (Solanki 1993; Lagg et al. 2010). A high concentration of these $k G$ magnetic flux tubes and sheets, such as in solar plage, can in turn alter the granulation pattern (Dunn and Zirker 1973) and produce smaller, abnormal granules (Title et al. 1987; Narayan and Scharmer|2010). Large flux tubes such as sunspots are able to inhibit the convective process effectively enough to cause the plasma within them to cool down to $4000 \mathrm{~K}$, making them appear dark compared to hot surrounding quiet Sun with its typical temperature of $5700 \mathrm{~K}$. However, convective processes within a sunspot are not entirely suppressed as is demonstrated by the bright penumbral filaments and umbral dots (Borrero and Ichimoto 2011).

The magnetic fields of sunspots and ARs are believed to be produced by a dynamo operating at the bottom of the convection zone at the tachocline (Charbonneau 2010). Flux tubes of the dynamo's toroidal magnetic field rise through the convection due to buoyancy, producing the familiar magnetic features observed on the solar surface. A turbulent dynamo operating close to the solar surface has also been proposed (Cattaneo 1999; Vögler and Schüssler 2007). Their contribution to the observed solar magnetic flux in particular to the internetwork flux currently forms a intensely discussed issue (de Wijn et al.2009).

The correlation between intensity and magnetic flux moves the study of solar magnetic fields beyond mere academic interests. While sunspots cause the familiar darkening of the solar surface at their location, the small flux tubes and sheets forming faculae/plage and the network appear brighter when compared to their surroundings. Due to the large number of faculae found in the vicinity of sunspots, an AR produces a net increase of the observed total irradiance of the Sun. During the maximum of a solar cycle the Sun's total solar irradiance will, therefore, be higher than during a solar minimum by $0.1 \%$ (Krivova et al. 2003, 2007). This difference in irradiance between solar maxima and minima is even greater, up to a factor of 100, when only the UV, EUV or radio wavelengths are considered (Domingo et al. 2009; Hathaway 2010).

\subsection{Zeeman effect}

Since the discovery of magnetic fields within sunspots, the Zeeman effect (Zeeman 1897) and its polarimetric signal remain the most widely used methods of determining magnetic fields on the Sun. The Zeeman effect occurs when the degeneracy of atomic energy state, $E_{J}$ disappears under the influence of an external magnetic field and becomes,

$$
E_{J, M}=E_{J}+\frac{\hbar e}{2 m_{e}} g M B
$$


where $\hbar=h / 2 \pi$ is the reduced Planck constant, $e$ is the electric charge, $m_{e}$ is the electron mass, $B$ is the magnetic field strength and $g$ is the Landé factor. $M$ is the magnetic quantum number and takes values of $M=-J, \ldots, 0, \ldots, J$. The quantum number $J$ describes the total angular momentum of a state, which couples the spin angular momentum, $S$, and the orbital angular momentum, $L$, of a state. For a given $J$ value a state can split $2 J+1$ components. Within Russel-Saunders $(L S)$ coupling the Landé factor, $g$, is calculated, when $J \neq 0$,

$$
g=\frac{3}{2}+\frac{S(S+1)-L(L+1)}{2 J(J+1)} .
$$

In the case that $J=0$, then also $g=0$ and no splitting is observed. The transition rules between two states are,

$$
\Delta M=M_{u}-M_{l}=0, \pm 1,
$$

where $l$ and $u$ refer to the lower and upper state of the transition, respectively. A transition where $\Delta M=0$ is a $\pi$ component, whereas a $\Delta M= \pm 1$ transition is a $\sigma$ component. Other transitions with a larger $\Delta M$ are forbidden as well as transitions between $J_{l}=J_{u}=0$ states. As a consequence of Eq. 1.3 the various components of a state do not necessarily have equal strength (del Toro Iniesta 2003).

For a normal Zeeman triplet $(J=1)$ the displacement, $\Delta \lambda_{B}$, of the $\sigma$ components from the $\pi$ component can be expressed by

$$
\Delta \lambda_{B}=\frac{e}{4 \pi c m_{e}} \lambda^{2} B\left(g_{l} M_{l}-g_{u} M_{u}\right),
$$

where $\lambda$ is the central wavelength of the absorption line in the absence of any splitting and $c$ is the speed of light. Note the $\lambda^{2}$ dependence, which indicates that, for a given magnetic field strength and Landé factor, the displacement is larger the longer the wavelength. If the absorption line under investigation shows abnormal Zeeman splitting, i.e. an energy state has multiple $\pi$ and $\sigma$ components, $g$ is replaced by an effective Landé factor $g_{\text {eff }}$, which is written,

$$
g_{\text {eff }}=\frac{1}{2}\left(g_{l}+g_{u}\right)+\frac{1}{4}\left(g_{l}-g_{u}\right)\left[J_{l}\left(J_{l}+1\right)-J_{u}\left(J_{u}+1\right)\right] .
$$

The normal Landé factor is sufficient only for a transition between $J=0$ and $J=1$ states. A transition between other $J$ states or when $L S$ coupling is no longer applicable, as is the case for atoms with a high nuclear charge, requires the use of $g_{\text {eff }}$. Outside of sunspots the separation of the $\pi$ and $\sigma$ components by solar magnetic fields is often weak enough that it effects nothing more than a broadening of the line, akin to Doppler broadening, $\Delta \lambda_{D}$.

The reliable retrieval of magnetic flux measurements outside of sunspots, when $\Delta \lambda_{B}$ is small, can be achieved by measuring the polarised light of the $\pi$ and $\sigma$ components. The Stokes parameters $Q$ and $U$ describe linearly polarized light, $V$ shows the circularly polarised light and $I$ displays the total intensity of the incoming light, so that

$$
\begin{gathered}
Q=I_{\text {lin }}(\phi=0)-I_{\text {lin }}(\phi=\pi / 2) \\
U=I_{\text {lin }}(\phi=\pi / 4)-I_{\text {lin }}(\phi=3 \pi / 4) \\
V=I_{\text {circ }}(\text { left })-I_{\text {circ }}(\text { right }) .
\end{gathered}
$$

Stokes $Q$ is the difference between two intensities that have passed through linear polarisers orientated at $\phi=0$ and $\phi=\pi / 2$, respectively. For a Stokes $U$ measurement the linear 
polarisers are orientated at $\phi=\pi / 4,3 \pi / 4$. Stokes $V$ is the difference between two intensities that have passed through a quarter-wave linear retarder and linear polariser orientated at $\pi / 4$ and $3 \pi / 4$, respectively. When a magnetised atmosphere is observed, then Stokes $Q, U$ and $V$ are related to Stokes $I$ by

$$
I^{2} \geq Q^{2}+U^{2}+V^{2}
$$

If the incoming light is unpolarised then $Q=U=V=0$. As indicated by acquisition of Stokes $Q$ and $U$ in Eq. 1.6, there is an inherent $180^{\circ}$ ambiguity in the azimuthal orientation of a magnetic field, calculated from the ratio between Stokes $Q$ and $U$ (Auer et al. 1977). For large magnetic fields the peaks of the Stokes $V$ profile are displaced according to Eq. 1.4, however, for small fields, when $\Delta \lambda_{B} \ll \Delta \lambda_{D}$ is satisfied, the peak separation remains constant and only the amplitude of Stokes $V$ grows. In this case the Stokes $V$ profile is, to first order, directly proportional to the partial derivative of Stokes $I$,

$$
V(\lambda) \approx \cos (\gamma) \Delta \lambda_{B} \frac{\partial I(\lambda)}{\partial \lambda}
$$

where $\gamma$ is the inclination of the magnetic field with respect to the line-of-sight. Eq. 1.8 further shows, whilst this approximation holds, that $V \propto B$. A similar approximation can be made for Stokes $Q$ and $U$ whereby $Q \propto B^{2}$ (Jefferies et al. 1989; Stix 2004).

\subsection{Hinode SOT/SP}

The Hinode satellite (Kosugi et al. 2007) was launched in September 2006, carrying three principal instruments an X-Ray Telescope, XRT (Golub et al. 2007), an EUV Imaging Spectrometer, EIS (Culhane et al.2007), and the Solar Optical Telescope, SOT (Tsuneta et al. 2008b; Suematsu et al. 2008; Ichimoto et al. 2008; Shimizu et al. 2008). It is in a sun-synchronous orbit allowing uninterrupted observations of the Sun for eight months, before entering an eclipse phase. Whilst the former two instruments are designed to study the corona and chromosphere, the SOT's aim is mainly to study the photosphere and its magnetic field. The SOT is a Gregorian telescope with a $50 \mathrm{~cm}$ aperture for its primary mirror with an effective focal length of $15.5 \mathrm{~m}$, giving it a diffraction limited resolution of $0.3^{\prime \prime}$ at $\approx 600 \mathrm{~nm}$. The SOT itself contains two instruments, a filtergraph, which is itself divided into a Narrow-band filtergraph, NFI, and a broadband filtergraph, BFI, and a spectropolarimeter, SP. While the BFI is designed to record only Stokes $I$ intensity images of the photosphere and chromosphere, both the NFI and the SP can record all four Stokes parameters, $I, Q, U$ and $V$. The filtergraph is also used to conduct focus campaigns to find the optimal focus position for both instruments. The NFI allows time series of the magnetic field to be taken and is therefore suited to study its morphology. The SP, a Littrow-Echelle spectrograph, has the superior spectral resolution at $30 \mathrm{~m} \AA$ with a noise level of $10^{-3} I_{c}$ for all four Stokes parameters at a exposure time of $4.8 \mathrm{~s}$ per slit position. It records the $6301.5 \AA,\left(g_{e f f}=1.5\right)$, and $6302.5 \AA,(g=2.5)$, Fe I line pair at 120 wavelength positions, ideally allowing the detection of a net vertical magnetic field of $5 G$ and a net horizontal magnetic field of $50 G$ (pixel average values for $4.8 s$ exposure time). The $y$-axis of the slit covers 162" on the solar disk and is aligned along the solar $N-S$ axis. This makes it the instrument of choice to study internetwork magnetic fields and also allows an 
accurate determination of any asymmetries in the Stokes spectra. All the investigations detailed in Chapters 2, 3 and 4 are predominantly based on SOT/SP data. The raw SOT/SP data is calibrated using the $s p \_p r e p$ routine (Lites and Ichimoto 2013), available in the Solarsoft Package to account for dark current and flat fielding, instrumental cross talk and orbital drift along the wavelength axis.

\subsection{Inversions}

The inversion of observed Stokes spectra is a common way to obtain information about an emitting atmosphere. It involves solving the radiative transfer equation (RTE) (del Toro Iniesta 2003),

$$
\frac{d \mathbf{I}}{d \tau_{c}}=\mathbf{K}(\mathbf{I}-\mathbf{S}),
$$

where $\mathbf{I}=(I, Q, U, V)^{\top}$ is the Stokes vector, $\tau_{c}$ is the continuum optical depth. $\mathbf{S}$ is the source function and in local thermodynamic equilibrium (LTE), whereby the local velocity distribution is Maxwellian and the level populations of a transition are governed by the Bolzmann and Saha equations, takes the form $\mathbf{S}=\left(B_{\lambda}(T), 0,0,0\right)$, where $B(T)$ is Planck's function. $\mathbf{K}$ is the propagation matrix,

$$
\mathbf{K}=\left(\begin{array}{cccc}
\eta_{I} & \eta_{Q} & \eta_{U} & \eta_{V} \\
\eta_{Q} & \eta_{I} & \rho_{V} & -\rho_{U} \\
\eta_{U} & -\rho_{V} & \eta_{I} & \rho_{Q} \\
\eta_{V} & \rho_{U} & -\rho_{Q} & \eta_{I}
\end{array}\right),
$$

where the absorption elements for a Zeeman-split line are (Unno 1956),

$$
\begin{gathered}
\eta_{I}=1+\frac{\eta_{0}}{2}\left[\phi_{0} \sin ^{2} \gamma+\frac{\phi_{+1}+\phi_{-1}}{2}\left(1+\cos ^{2} \gamma\right)\right], \\
\eta_{Q}=\frac{\eta_{0}}{2}\left[\phi_{0}+\frac{\phi_{+1}+\phi_{-1}}{2}\right] \sin ^{2} \gamma \cos 2 \chi, \\
\eta_{U}=\frac{\eta_{0}}{2}\left[\phi_{0}+\frac{\phi_{+1}+\phi_{-1}}{2}\right] \sin ^{2} \gamma \sin 2 \chi, \\
\eta_{V}=\frac{\eta_{0}}{2}\left[\phi_{-1}-\phi_{+1}\right] \cos \gamma,
\end{gathered}
$$

and the dispersion elements are (Rachkovsky 1962),

$$
\begin{gathered}
\rho_{Q}=\frac{\eta_{0}}{2}\left[\psi_{0}-\frac{1}{2}\left[\psi_{+1}+\psi_{-1}\right]\right] \sin ^{2} \gamma \cos 2 \chi, \\
\rho_{U}=\frac{\eta_{0}}{2}\left[\psi_{0}-\frac{1}{2}\left[\psi_{+1}+\psi_{-1}\right]\right] \sin ^{2} \gamma \sin 2 \chi, \\
\rho_{V}=\frac{\eta_{0}}{2}\left[\psi_{-1}-\psi_{+1}\right] \cos \gamma .
\end{gathered}
$$

The variables $\gamma$ and $\chi$ are the line-of-sight inclination and azimuth, respectively, and $\eta_{0}$ is the ratio between the line and the continuum absorption coefficients. The absorption profiles are $\phi_{\alpha}$, where $\alpha$ takes \pm 1 for the $\sigma$ components of the absorption line and 0 for the $\pi$ component, and the dispersion profiles are expressed by $\psi_{\alpha} . \phi_{\alpha}$ and $\psi_{\alpha}$ are Voigt and Faraday-Voigt functions, respectively, which take into account a line-of-sight velocity 
shift, Doppler broadening of thermal motions and line dampening due to collisions of an absorption line.

A widely employed solution to Eq. 1.9 is one where $\mathbf{K}$ corresponds to the Milne-Eddington (ME) atmosphere, whose key feature is the invariance of all the matrix elements of $\mathbf{K}$ with optical depth and the source function takes the form $\mathbf{S}=\left(S_{0}+S_{1} \tau, 0,0,0\right)^{\top}$. For such an atmosphere an analytical solution for Eq. 1.9 exists, whilst solutions for more complex and more realistic atmospheres can only be obtained by computationally expensive numerical means.

The Stokes-Profiles-INversion-O-Routines (SPINOR) code (Frutiger et al. 2000), based on the STOPRO routines (Solanki 1987), allows the use of more complex atmosphere than ME atmosphere to fit observed Stokes profiles. The code employs the MULTI 2.2 opacity package (Carlsson 1986) to obtain the continuum absorption coefficients, taking into account contributions from $\mathrm{H}, \mathrm{He}, \mathrm{H}^{-}, \mathrm{He}^{-}, \mathrm{H}_{2}^{+}, \mathrm{H}_{2}^{-}$and other donor species. Rayleigh scattering by monatomic and diatomic hydrogen and Thomson scattering are also considered. The broadening of spectral lines by collisions with neutral hydrogen is taken into account using the ABO theory (Anstee and O'Mara 1995; Barklem and O'Mara 1997; Barklem et al. 1998). The RTE is typically solved using the comparatively quick Hermitian approach (Bellot Rubio et al. 1998), but the DELO method (Rees et al. 1989) can also be used. The Stokes spectra synthesised by SPINOR are fitted iteratively to observed spectra using a Levenberg-Marquardt algorithm (Press et al. 1986) with a $\chi^{2}$ merit function. Spatially coupled 2D inversions of Stokes spectra using the SPINOR code have been made possible by van Noort (2012).

\subsection{Internetwork magnetic fields}

Magnetic fields in the internetwork have been, certainly from a historical perspective, investigated the least when compared to magnetic fields in ARs or the network. This lack of characterisation of internetwork magnetic fields can be attributed to the inherent difficulty associated with observing them, however, an initial attempt to find turbulent magnetic fields was made by Unno (1959).

\subsubsection{Observations}

The investigation performed by Livingston and Harvey (1975) was the first direct observation of internetwork magnetic fields in Stokes $V$ magnetograms, which is more than half a century after the discovery of magnetic fields in sunspots (Hale 1908). Later ground based observations (Zirin|1987; Martin 1988; Lin 1995; Lin and Rimmele|1999; Lites et al. 1996; Solanki et al. 1996b; Harvey et al. 2007) confirmed the existence of magnetic fields in the internetwork. However, the inherent turbulent nature of internetwork magnetic fields (Pietarila Graham et al. 2010) and the comparatively small fluxes involved make them difficult to detect when employing the Zeeman effect. The Stokes $Q, U$ or $V$ polarisation signals are prone to cancellation effects stemming from a lack of spatial resolution, which reduce the observed signal and thereby reduce the apparent field strength. Therefore, in the case of Stokes $V$ two equal magnetic fields of opposite polarity within an unresolved structure will cause a net polarisation signal of zero, leading to the erroneous conclusion 
that no magnetic fields are present. This cancellation effect can occur for Stokes $Q$ or $U$ as well, but it requires magnetic fields orientated perpendicular to each other, which is less common in photosphere due to the convection pattern. The close proximity of opposite polarity magnetic fields is common in the turbulent internetwork. In order to make such magnetic fields visible in Stokes $Q, U$ or $V$, a high spatial resolution needs to be achieved. However, apart from a high spatial resolution, a high polarimetric sensitivity is also required to detect magnetic flux patches. In the case of SOT/SP a continuum noise level in the order of $10^{-3} I_{c}$ is necessary, given the diffraction limited observations have a spatial resolution $0 . " 32$, to detect features down to $10^{16} M x$, which allows the detection of the largest flux patches in the internetwork. For comparison pores and sunspots have fluxes of $10^{19} M x$ or greater. A high sensitivity is even more essential when measuring Stokes $Q$ or $U$, since $Q \propto B^{2}$, which makes the detection of weak horizontal fields very challenging. A short exposure time, whilst achieving a $10^{-3} I_{c}$ noise level, is also desirable, since internetwork flux patches have lifetimes of only minutes or less (Lin and Rimmele 1999; Lites et al. 1996).

The internetwork is most commonly investigated using absorption lines in the visible, such as the $630 \mathrm{~nm}$ line pair $\left(g_{\text {eff }}=1.5\right.$ and $\left.g=2.5\right)$, or in the infrared, such as the $1.56 \mu \mathrm{m}$ line $(g=3)$. Absorption lines in the visible have the advantage of potentially allowing observations at a higher spatial resolution than observations using an infrared line, given an equal telescope aperture. However, the atmospheric degradation of the measurements affecting ground-based observations is greater for shorter wavelengths when no adaptive optics system is employed (Roddier 2004), leading often to a similar effective spatial resolution. Infrared lines on the other hand are very sensitive especially to weak magnetic fields, since the Zeeman splitting in Eq. 1.4 has a $\lambda^{2}$ dependence, while the Doppler broadening is only linearly dependent on $\lambda$. Therefore, a large fraction of magnetic fields detected using infrared lines remain below the noise level in observations using visible lines (Bellot Rubio and Collados 2003).

Given the comparative difficulty in measuring internetwork magnetic fields directly, proxy magnetometry has been employed to estimate the average magnetic field strength in the internetwork. Bright points on the solar surface are a common sight in the network (Lites et al. 1993; Muller et al. 2000) and active regions (Möstl et al. 2006) and a few can also be seen in the internetwork (de Wijn et al.2005; Jafarzadeh et al.2013). Since these bright points in the photosphere are of magnetic origin (Riethmüller et al. 2010), their density across the observed region can serve as an indicator of the average magnetic field strength (Berger and Title 2001; Utz et al. 2013). Based on the measured bright point density, realistic MHD simulations have been calibrated to determine what average magnetic field needs to be injected into the simulation box to reproduce the observed bright point density (Keys et al.2011). However, the detection of these bright points is highly dependent on the achieved spatial resolution, making it difficult to identify the true bright point density. The Hanle effect presents another way of measuring magnetic fields in the photosphere using polarimetry. Unlike the Zeeman effect the polarimetric signal from the Hanle effect does not suffer from signal cancellation caused by opposite polarity magnetic fields within the resolution element (Stenflo 1982; Faurobert-Scholl 1996). This allows the retrieval of the true average magnetic field strength within the resolution element. Furthermore, it is most sensitive to weak magnetic fields, thus complementing magnetic field strengths obtained via the Zeeman effect (Solanki 1993; de Wijn et al. 2009). 
The study of the internetwork using observations is only at its very beginning, which is demonstrated most clearly by the divergence in the estimation of the average unsigned magnetic flux density in the internetwork. Wang et al. (1995) retrieve a value of only $4.8 G$ at a spatial resolution of $>1$ ". At a spatial resolution of $\approx 1$ " the average unsigned flux increases to around $10 G$ (Lites 2002; Sánchez Almeida and Lites 2000). Domínguez Cerdeña et al. (2003) using speckle reconstructed images at $630 \mathrm{~nm}$ achieved a spatial resolution of 0.5 " and reported an unsigned flux density of $20 \mathrm{G}$. A similar flux density was found by Khomenko et al. (2003) using the $1.56 \mu \mathrm{m}$ line. Taking advantage of the seeing free data offered by SOT/SP Lites et al. (2008) aboard the Hinode satellite found flux densities of $11 G$ and $55 G$ for vertical and horizontal magnetic fields respectively. The high flux density reported for horizontal magnetic fields seems to support some of the high flux densities of $60-130 G$ reported by Trujillo Bueno et al. (2004); Bommier et al. (2005) using the Hanle effect, although lower flux densities have also been reported (Stenflo et al. 1998). It appears unavoidable that more modern telescopes with higher spatial resolution will produce yet higher magnetic flux densities in the internetwork (Domínguez Cerdeña et al. 2006; Pietarila and Pietarila Graham 2013).

After the discovery of magnetic fields in the internetwork, the question of whether the magnetic field is organised in predominantly $k G$ flux tubes/sheets, like ARs and the network, or in diffuse $h G$ structures soon arose. Whilst $k G$ features in the form of bright points no doubt exist in the internetwork (Mehltretter 1974), although a confirmation for this was not provided until the work of Lagg et al. (2010). Observations using Stokes $I$ and $V$ spectra of the $1.56 \mu m$ line performed by Lin and Rimmele (1999); Khomenko et al. (2003) found a predominance of $h G$ fields near the equipartition field strength in the internetwork. Investigations using multi-component ME inversions of the 630 $\mathrm{nm}$ line instead detected a prevalence of $k G$ in the internetwork (Domínguez Cerdeña et al. 2003; Lites 2002; Socas-Navarro and Lites 2004). Several studies involving the simultaneous recording of the internetwork using both the $630 \mathrm{~nm}$ line pair and $1.56 \mu \mathrm{m}$ line were conducted by Khomenko et al. (2005) and Martínez González et al. (2008). While Khomenko et al. (2005) demonstrated the superior sensitivity of the infrared line in detecting the weak fields, Martínez González et al. (2008) showed that a simultaneous inversion of all three lines yielded predominantly $h G$ in the internetwork. This supported earlier results from Martínez González et al. (2006) that inversions results based on the Stokes $I$ and $V$ spectra of the $630 \mathrm{~nm}$ lines alone are not unique and potentially lead to a erroneous conclusion of a predominance of $k G$ in the internetwork. Internetwork observations obtained by the SOT, have yielded a predominance of $h G$ fields based on the inversion of the $630 \mathrm{~nm}$ lines alone (Orozco Suárez et al.2007). The prevalence of $h G$ fields in the internetwork (Lin 1995) supports results obtained by Solanki et al. (1996b) using the $1.56 \mu \mathrm{m}$ line, which indicated that the convective collapse mechanism producing $k G$ features is less effective in weak flux concentrations as predicted by Venkatakrishnan (1986).

Starting with the centre-to-limb investigation performed by Martin (1988), it has been suggested that the internetwork is composed of a myriad of magnetic loops, which do not reach into the chromosphere (Martínez González et al. 2007; Martínez González and Bellot Rubio 2009; Wiegelmann et al.2010). Stokes $Q$ and $U$ measurements of the internetwork at the disc centre performed by Lites et al. (1996) and Harvey et al. (2007) also detected horizontal fields in the internetwork. However, it was seeing-free and stable data offered by SOT that allowed the horizontal fields in the internetwork to be investigated 
in detail. Ishikawa and Tsuneta (2010) showed that the internetwork contains numerous patches of horizontal magnetic fields near the edges of granules, which are characterised by a short lifetime. The azimuthal orientation of these patches appears to be isotropic (Ishikawa and Tsuneta 2010) and their emergence rate appears to be the same, both within the internetwork and near plage (Ishikawa and Tsuneta 2009). Furthermore they have been found over the entire solar surface (Tsuneta et al. 2008a). The results presented by Danilovic et al. (2010a) using IMaX (Martínez Pillet et al.2011) on Sunrise (Barthol et al. 2011) confirmed both the short lifetime of these horizontal features and that they often are seen to be associated with a patch of vertical fields in an intergranular lane. Lites et al. (2008) analysing SOT/SP data concluded that in the internetwork the flux density of horizontal magnetic fields exceeds the flux density of vertical fields, leading to a dominance of horizontal fields in the internetwork. The dominance of horizontal fields was further suggested by the inversion of SOT/SP spectra performed by (Orozco Suárez et al. 2007), which returned highly inclined fields. However, this predominance of horizontal fields has been put into question by Borrero and Kobel (2012, 2013), who showed that the inversion of noisy Stokes $Q$ and $U$ will inevitably and erroneously lead to a prevalence of horizontal magnetic fields. Instead these authors as well as Asensio Ramos (2009) prefer an isotropic distribution of inclinations. Stenflo (2013) has argued that the internetwork is composed of mainly vertical magnetic fields like the network.

\subsubsection{Origin}

While it could be argued that magnetic fields found in the quiet Sun are merely remnants of ARs shredded by the photospheric convection, it was soon realised that the total unsigned flux in the quiet Sun at any given time exceeds the total unsigned flux found in ARs (Zirin 1987; Petrovay and Szakaly 1993). Harvey et al. (1975); Harvey (1993); Hagenaar (2001) showed that the network is formed by the emergence of smaller ephemeral regions, which are thought to be generated by the same global dynamo process responsible for generating ARs. Both Harvey (1993) and Hagenaar (2001) reported that ephemeral regions $>10^{18} M x$ still follow Joy's law and their emergence rate shows a solar cycle dependence albeit weaker than for ARs. They also have a preferred $E-W$ orientation similar to ARs. Emerging flux patches $<10^{18} M x$ no longer show a dependence on Joy's law and have an isotropic orientation (Ishikawa and Tsuneta 2010), which could suggest that these small flux patches are subjected to a remixing and recycling by the convection (Ploner et al. 2001), but nonetheless ultimately a product of the global dynamo.

An alternative source for the internetwork flux could be a local dynamo process operating close to the solar surface. This dynamo process is driven by the turbulent motions of the plasma commonly found in intergranular lanes as well as on larger scales but not by rotation as the global dynamo. Initial local dynamo simulations were performed by Cattaneo (1999), but it was the simulations run by Vögler et al. (2005), taking into account both the radiative heat loss at the solar surface and the downward pumping of magnetic flux (Stein et al. 2003) due to the convection, which showed that a sustainable local dynamo process is feasible near the solar surface. The magnetic fields produced by this dynamo also have several qualitative properties already observed in the internetwork, such as the high mixing of opposite polarities without a significant dominance of either polarity, a 
comparatively high horizontal flux density and, being independent from the global dynamo, no preferred orientation or adherence to Joy's law of the generated magnetic fields.

Given this apparent suitability of the local dynamo for being the source of the internetwork flux, a considerable effort has been made to support the existence of such a dynamo through observational evidence. Danilovic et al. (2010b) compared a internetwork SOT/SP observation with several local dynamo simulation runs using a range of values for $R_{m}$ and found the simulation results to be compatible with the observation. A similar conclusion was reached by Schüssler and Vögler (2008) who also found a similar ratio between the horizontal and vertical magnetic flux as reported by Lites et al. (2008). The constant apparent emergence frequency of small horizontal flux patches, be it in the internetwork or near plage as reported by Ishikawa and Tsuneta (2009), can also be seen as an indication for local dynamo action occurring over the entire surface. This idea is supported further by the results of Ito et al. (2010); Shiota et al. (2012) who analyzed the solar polar magnetic fields, and found, apart from magnetic patches with $k G$ fields forming part of the global dipole field, a myriad of turbulent, transient and weak magnetic fields. Lites (2011) argued, based on SOT/SP results, that the observed balance of the signed magnetic flux in the internetwork is another indication of a local solar dynamo producing the majority of the internetwork magnetic fields. Criticism against a local dynamo scenario has mainly been directed at the limitation of MHD simulations to fully reproduce solar convective processes (Stein et al.2003), but also at determining the true preferred inclination of internetwork magnetic fields. In particular Stenflo (2013) has argued repeatedly that the internetwork too is predominantly composed of vertical magnetic fields, which speaks against the horizontal fields produced by a local dynamo. Furthermore, Stenflo (2012), using MDI magnetograms, has indicated that the flux density contributed by a local dynamo can only amount to $3 G$ and, therefore, the currently observed magnetic fields are either directly or indirectly produced by the global dynamo.

The distribution of magnetic flux on the solar surface, down to the current cut-off of $10^{15} M x$, follow a single power law (Parnell et al. 2009; Thornton and Parnell 2011; Iida et al. 2012), indicating that dynamo action could occur on all convection scales rather than as one small-scale and one large-scale dynamo. Guglielmino et al. (2012) reported, by analysing a bi-pole carrying $10^{17} M x$ of flux using IMaX data, that the emergence rate of such structures over the field-of-view was lower that than expected. Furthermore, the contribution of the internetwork flux to the network is still unclear. Hagenaar (2001) showed that the network is formed by ephemeral regions, which emerge at a rate with which the entire network flux is replaced every $8-19$ hours and that the emergence rate increases exponentially with decreasing size. However, despite the high emergence of new flux in the internetwork (Zirin 1987) the turbulent nature of the plasma causes a large fraction this flux to be cancelled before it can be swept to edge of a supergranule. Ishikawa and Tsuneta (2011) have observed that some emerging flux in the internetwork is nonetheless being advected to supergranular boundaries and thereby able to contribute to the network.

Despite the numerous investigations over the last two decades into the characteristic properties of the internetwork magnetic fields, many issues remain unresolved, such as their origin, the average unsigned flux density, the ratio between horizontal and vertical flux densities, or whether there is any cycle dependence in the internetwork magnetic fields. 
This last issue has so far received the least amount of attention, in particular due to the limited availability of data suitable for such an investigation. With the launch of the Hinode satellite it has become possible to investigate this issue in detail. An invariant internetwork magnetic flux with respect to the solar cycle would be another support for a local dynamo being responsible for producing the bulk of the observed internetwork magnetic fields. Furthermore, it may also then serve as an indication that the solar surface would even in times of low activity, such as the Maunder minimum, not be devoid of magnetic fields. Chapter 2 will attempt to provide an answer to this question.

\subsection{Magnetic flux tubes}

The study of magnetic flux tubes/sheets deserves particular attention, since these comparatively small photospheric structures form the foot points of coronal magnetic fields upon which the upper layers of the solar atmosphere are organised. The largest flux tubes on the Sun are sunspots and pores, the largest of which can be spatially resolved even with a small telescope. It would, however, be bold to say that the study of sunspots and pores, due to their size, is nearing completion. In particular sunspots host a remarkable degree of fine structure in the photosphere, such as penumbral filaments or umbral dots and current observatories have only begun to resolve these features. However, the majority of flux tubes in the photosphere are far smaller than sunspots, having typical diameters of only $100 \mathrm{~km}$ to $300 \mathrm{~km}$, which turns the analysis of these features, to this day, into the seemingly counterintuitive exercise of identifying the properties of an object one can't even see properly.

The analysis of these small magnetic flux tubes/sheets can only be conducted by analysing the Zeeman effect-induced polarization of the $\sigma-$ and $\pi$-components of an absorption line. Unlike the intensity recorded by Stokes $I$, the Stokes $Q, U$ and $V$ profiles only contain information about the magnetised atmosphere, even when it is not spatially resolved. The first analysis of Stokes $V$ magnetograms was carried out by Babcock and Babcock (1955) and found magnetic fields outside of sunspots albeit with nowhere near the $k G$ field values known to exist in sunspots. Later investigations soon discovered that the mean magnetic field strength correlated with the spatial resolution, tempting the question of what was the true field strength of magnetic fields outside sunspots and how was it organised. Stenflo (1973) was the first to show that the magnetic fields are concentrated in a small area, where they attain $k G$ field strengths, while the atmosphere between these concentrated structures is almost field free. This result was obtained using the line ratio technique, whereby the ratio of the Stokes $V$ amplitudes of the $5250 \AA$ and $5247 \AA$ lines is taken. These two lines are remarkable since they are identical except for their Landé factors, which makes the ratio of their Stokes $V$ signals only dependent on the magnetic field.

After the discovery of these $k G$ magnetic fields a physical mechanism needed to be found, which would be capable of concentrating magnetic fields to $k G$ values. Since magnetic fields in the photosphere are frozen-in-flux due to the high magnetic Reynolds number, any emerged field will be swept into an intergranular lane within a few turnover cycles (Schüssler 1986). This process concentrates the magnetic field in these lanes up to the 
equipartition field strength, which can be calculated using,

$$
\frac{B^{2}}{8 \pi}=\frac{1}{2} \rho v^{2}
$$

where $B$ is the magnetic field strength, $\rho$ is the plasma density and $v$ is the velocity. Taking typical values for the photosphere an equipartition field strength of $\approx 400 G$ is obtained. This value is, however, too small by far to account for the observations and, therefore, another mechanism is need to concentrate the field to $k G$ values. The convective collapse mechanism occurs because the convection is hindered due to the presence of $h G$ magnetic fields, causing the plasma to cool radiatively and then rapidly sink due to gravity (Parker 1978). The plasma in the magnetized region thereby becomes rarefied and gets concentrated to maintain the pressure balance with its surroundings,

$$
\frac{B^{2}}{8 \pi}+P_{i n}=P_{e x}
$$

where $B$ is the magnetic field strength and, $P_{i n}$ and $P_{e x}$ are the internal and external gas pressures, respectively. From this equation it clear that $P_{i n}<P_{e x}$, causing the plasma inside the tube to become optically thick at a lower geometrical height than the surrounding quiet Sun and effectively allows an observer to look deeper into the Sun. This effect is analogous to the Wilson depression observed in sunspots. Furthermore, the $k G$ magnetic field suppresses convective processes within the tube, the plasma $\beta<1$, and thus prevents the rise of hot plasma within the tube, which would reverse the convective collapse mechanism. A necessary requirement to verify the convective collapse mechanism is the observation of the fast downflows during the concentration of the magnetic field. However, the high spatial resolution necessary to observe this process meant that only few direct observations of it exist (Nagata et al.2008) so far.

A common way to model $k G$ magnetic features involves the thin flux tube approximation. In this magnetohydrostatic (MHS) model, the atmosphere is in hydrostatic equilibrium and the radius of the tube is assumed to be small compared to the pressure scale height. The thin flux tube approximation is obtained by performing a Taylor expansion upon the MHS equations (Pneuman et al. 1986; Ferriz-Mas et al. 1989) and only taking the zeroth order terms of the expansion into account (Defouw 1976). This solution ignores any magnetic curvature forces or twist and assumes that the electrical resistivity at the boundary of the tube is zero. Due to the hydrostatic equilibrium the external and the internal gas pressure drops exponentially (for an isothermal atmosphere), leading to an expansion of the tube with height and consequently a reduction of $B$. The total magnetic flux of the tube is, however, conserved at all heights. The zeroth order thin flux tube model has been shown to be in good agreement with observations (Zayer et al. 1989). Higher order thin flux tube approximations have also been considered (Pneuman et al. 1986) and Yelles Chaouche et al. (2009) found that a second order approximation is adequate to model flux tubes produced in MHD simulations. Given equal external atmospheres flux tubes of different sizes, i.e. field strenghts, have the same relative expansion with height and Solanki et al. (1992b, 1999) showed that even the expansion of sunspots can be modelled using a thin tube approximation.

The $k G$ nature of the magnetic fields has by now been confirmed many times (Rabin 1992, Zayer et al. 1990; Rüedi et al. 1992; Stenflo and Harvey 1985; Martínez Pillet et al. 1997) 
using many different Zeeman effect based techniques, one of which is the inversion of measured Stokes spectra by a least-squares fitting algorithm using ME (Martínez Pillet et al. 1997; Narayan and Scharmer 2010) or more complex atmospheres (Del Toro Iniesta and Ruiz Cobo 1996). This technique was pioneered by Auer et al. (1977) and has by now become a standard tool for analysing the photosphere. The aim of this technique is to retrieve information about the magnetised atmosphere beyond the mere measurement of the field strength. All four Stokes spectra, given adequate spectral resolution, show various asymmetries such as in amplitude and area, which indicate the existence of velocity and/or magnetic field gradients along the line of sight (Solanki 1993). Whilst these asymmetries offer a possibility to determine the sub-resolution properties of flux tubes, many asymmetries can be caused by various combinations of effects and, therefore, demand the simultaneous observation and inversion of many spectral lines in order to accurately retrieve the properties of the flux tube. The widths of the Stokes $Q, U$ or $V$ spectra may even indicate the sub-resolution spatial distribution of the magnetic field.

The plasma in the photosphere is close to being in local thermodynamical equilibrium (LTE), which allows the use of Planck's black body radiation formula in relating the measured intensity with the temperature of the plasma. Therefore, the familiar bright granules are hotter than the darker and cool intergranular lanes. Small $k G$ magnetic features, however, appear as bright as granules despite being located in intergranular lanes, which are permeated by cool downflowing plasma. This seemingly paradoxical observation can be explained when considering the $\tau=1$ surface where the plasma becomes optically thin. While the geometrical height at which $\tau=1$ is wavelength dependent i.e. longer wavelengths probe deeper into the atmosphere, a commonly used $\tau=1$ surface is at $5000 \AA, \tau_{c}$. The $\tau_{c}$ surface is not formed at a common geometrical height across the solar surface due to the temperature and electron pressure variations of the solar plasma. In a $k G$ feature the plasma density is markedly lower than in the surrounding quiet Sun at a given geometrical height, causing the $\tau_{c}$ surface to be located deeper inside the Sun and thus exposing the comparatively hotter interior. The $k G$ features appear brightest in the continuum when viewed at an angle, which can be observed easily when performing a centre-to-limb investigation of these features. At $\mu$ values of 0.3-0.4, where $\mu=\cos (\theta)$ and $\theta$ is the heliocentric angle, the brightness is greatest (Ortiz et al. 2002; Kobel et al. 2009), since the depression in the $\tau_{c}$ surface allows the observer to see the hot walls (Spruit 1976) of the $k G$ features. Small $k G$ features will also appear bright when viewed at the disk centre. Near $\mu=1$ the relation between the continuum intensity and the magnetic field strength is complex, since for small field strength the intensity steadily increases, but then starts to decrease again (Keller 1992, Kobel et al. 2011) to form dark pores. Furthermore, Muller (1983); Mehltretter (1974) showed that the intensity can vary over a matter of minutes indicating that the granular convection may influence the intensity of $k G$ features (Berger and Title 1996). The lateral radiation from the hot walls cause the plasma inside the feature to heat up producing the observed relative brightening. The lateral heating causes the plasma inside the feature to have a temperature increase of $300-400 K$ (Solanki 1986; Solanki and Brigljevic 1992; Keller et al. 1990) when compared to the Harvard Smithsonian Reference Atmosphere (HSRA) (Gingerich et al. 1971) commonly used to model the surrounding quiet Sun. Flux tubes/sheets forming the network are hotter at $\tau_{c}$ than tubes/sheets found in plage (Solanki 1986), since their high density in plage causes abnormal cooler granules to form (Dunn and Zirker 1973; Title et al. 1989), which cannot 
heat the tube/sheet as effectively (Ortiz et al. 2002; Kobel et al. 2011).

There are also correlations between chromospheric intensity and photospheric magnetic fields. The most famous of these are the Ca II H and $\mathrm{K}$ line core intensity correlation with the network and plage magnetic fields (Frazier 1971; Skumanich et al. 1975; Schrijver et al. 1989; Utz et al. 2013) but also Mg I b lines (Beckers et al. 1976). When a spatial resolution better than $1 "$ is achieved then the correlation starts to break down and Rutten and Uitenbroek (1991) showed that some Ca II K brightenings are non magnetic in origin (Rezaei et al.2007). A close connection also appears to exist between $H \alpha$ filigrees and the magnetic fields on the photosphere (Dunn and Zirker 1973). Observations of the corona no longer show a correlation with the photospheric magnetic field (Sheeley et al. 1975), but rather trace magnetic loops connecting patches of opposite polarity magnetic fields. Nonetheless, both the chromospheric and coronal activity appears closely related to the photospheric field (see Beckers 1981; Mackay and Yeates 2012).

Apart from the $5250 \AA$ line pair (Stenflo 1973), the $6302 \AA$ line pair as well as other red Fe I lines (Wiehr 1978) in the visible have been used to determine the field strength of $k G$ magnetic features. The typical magnetic field values are between $1200 G$ and $1500 G$ at $\tau_{c}$. These field strengths are supported by similar investigations using infrared lines, such as at 1.56 $\mu \mathrm{m}$ (Zayer et al. 1989; Rüedi et al.1992; Rabin 1992). Due to the strong Zeeman splitting of these lines the magnetic field strength range in plage and network areas could be constrained. The magnetic field values of only $200 G$ in plage obtained by Zirin and Popp (1989) using the $12 \mu \mathrm{m}$ line were found to be in agreement with $k G$ thin flux tube models, after its formation height in the upper photosphere had been established (Bruls and Solanki 1995).

Absorption lines formed deeper in the photosphere such as the $1.56 \mu \mathrm{m}$ line generally show higher average field strengths (Zayer et al. 1989; Rüedi et al.1992), implying that the $k G$ features do expand and consequently a magnetic field gradient is present. The area asymmetry routinely observed in Stokes $V$ makes the same implication, whilst also indicating a velocity gradient (Solanki 1993; Martínez González et al.|2012). However, the accurate retrieval of this magnetic field gradient in plage or network areas is difficult due to the limited available spatial resolution. A centre-to-limb study performed by Martínez Pillet et al. (1997) indicates a gradient of $-3 \pm 1 G / \mathrm{km}$ and agrees with the $4 \mathrm{G} / \mathrm{km}$ gradient obtained from flux tube models (Solanki 1986; Solanki and Brigljevic|1992). However, this measurement is contaminated by the mixture of horizontal and vertical magnetic fields due to the viewing geometry. Modern inversion codes usually employ ME atmospheres to fit observed Stokes spectra, which intrinsically assume a height invariant magnetic field strength and therefore prevent the determination of the magnetic field gradient. Whilst more complex inversion codes such as Stokes Inversion based on Response functions (SIR) (Ruiz Cobo and del Toro Iniesta 1992) or SPINOR (Frutiger et al. 2000) can obtain magnetic field gradients, the number of extra free parameters as well as the necessity of a high spectral resolution, a high signal-to-noise ratio and straylight model continue to make the retrieval of such a gradient challenging. The geometric expansion of $k G$ magnetic features has also been studied by analysing the change of the Stokes $V$ area asymmetry from the disk centre to the limb (Buente et al. 1993; Martínez Pillet et al.|1997). Rezaei et al. (2007) found by studying the change of the area asymmetry across a $k G$ feature observed at the disk centre that it is in agreement with an expanding flux tube. A similar result was obtained by Martínez González et al. (2012) using IMaX data. 
The measurement of line-of-sight velocities in the photosphere forms a major part in the analysis of the properties of the Sun and its various features. The most straight forward way of measuring an absolute line-of-sight velocity is to measure the displacement of the core in Stokes $I$ of a line with respect to a reference wavelength. The calibration of such a reference wavelength is usually performed by the simultaneous measurement of a telluric absorption line, caused by water vapour in the Earth's atmosphere, followed by a correction for the motion of the Earth relative to the Sun, the Sun's and Earth's rotation as well as the gravitational redshift. A potential velocity gradient along the line-of-sight can be measured with the help of a bisector analysis of the Stokes $I$ absorption line. Since the core of an absorption line is formed higher in the atmosphere than its wings, which are formed near the continuum formation height, a relative shift between the wings and the core, i.e. an asymmetry in the line, can point to a velocity gradient. These two methods, however, only give the correct information about the line-of-sight velocity if the features in question are spatially resolved. If this is not the case then an asymmetry can also be produced by two (or more) atmospheres within the resolution element that have different temperatures, such as granules and intergranular lanes (Schröter 1957; Dravins et al. 1981). As the $k G$ features in plage or the network are generally not resolved an alternative to Stokes $I$ must be used in order to determine the line-of-sight velocities within a $k G$ feature. A common way to measure the velocity within an unresolved $k G$ feature is to use the Stokes $V$ zero crossing wavelength (Solanki 1986). Since Stokes $V$ only carries information about the magnetic part of an unresolved feature, a spurious velocity measurement due to the mixing of two atmospheres can be avoided. Needless to say that should the resolution element itself contain more than one magnetic feature then the Stokes $V$ crossing wavelength may also give only a weighted average line-of-sight velocity. Furthermore, a high spectral resolution and a low noise level are desirable when determining this wavelength. Whilst the Stokes $V$ zero crossing wavelength will yield only a single velocity measurement, both the blue-red amplitude and area asymmetries routinely observed in Stokes $Q, U$ and $V$ spectra strongly suggest the presence of velocity gradients within $k G$ features. In particular the area asymmetry suggests that a velocity gradient alone is not sufficient but that also magnetic field, inclination or azimuth gradients are required to fully reproduce the atmospheric conditions in such a feature (Solanki 1993).

The idea of stationary mass motions within $k G$ features has attracted considerable attention, since such flows could be a photospheric signature of chromospheric or coronal activities. Early Stokes $I$ observations did show downflows in low spatial resolution plage observations (Simon and Leighton 1964; Skumanich et al. 1975). Low spectral resolution Stokes $V$ zero crossing measurements further suggested downflows up to $1.6 \mathrm{~km} / \mathrm{s}$ within $k G$ features (Giovanelli and Slaughter 1978). Later Stokes $V$ analysis performed at a higher spectral resolution, allowing a more precise determination of the Stokes $V$ crossing wavelength, however, found that on average only downflows of $200 \mathrm{~m} / \mathrm{s}$ exist in $k G$ features (Solanki 1986; Fleck and Schmitz 1991; Martínez Pillet et al. 1997; Langangen et al. 2007). The ever present amplitude and area asymmetries observed in Stokes $V$ needed to be explained, whilst keeping the plasma inside the $k G$ feature almost at rest. A combination of $\approx 1 \mathrm{~km} / \mathrm{s}$ downflows surrounding the feature, reminiscent of cool intergranular lanes, and a magnetic field gradient was needed to reproduce the observed spectra (Grossmann-Doerth et al. 1989; Solanki 1989). Recent MHD simulations support this scenario (Steiner et al. 1996). An exception to the absence of stationary flows within $k G$ features come in the form of 
siphon flows (Rüedi et al. 1992) between two opposite magnetic polarities. Non stationary velocities in $k G$ features hinting at waves within the features have been analysed using both Stokes $I$ and $V$ (Fleck and Schmitz 1991; Deubner and Fleck 1990). Furthermore, it appears that the correct ratio between the amplitude and area asymmetries in Stokes $V$ spectra can only be reproduced if non stationary mass motions are introduced in flux tube models (Solanki 1989; Grossmann-Doerth et al. 1991). Bellot Rubio et al. (1997) argued, using a inversion results, which included atmospheric gradients, that stationary mass motions inside flux tubes can successfully reproduce all the asymmetries in observed Stokes spectra. According to the inversion, strong downflows within the flux tubes occurred particularly in deeper layers of the atmosphere (Bellot Rubio et al. 2000). However, Frutiger and Solanki (1998, 2001) pointed out that other thin flux tubes models involving oscillations within the flux tube were equally capable of reproducing the observed asymmetries. Furthermore, if mass conservation was imposed then only the oscillatory model could reproduce the observed spectra.

Information about the inclination and azimuth of the magnetic field can only be obtained when all four Stokes parameters have been measured. Even when both Stokes $Q$ and $U$ have been recorded the inherent $180^{\circ}$ ambiguity in the azimuth needs to be resolved before a unique orientation of the magnetic field vector can be determined (Leka et al. 2009). Due to the lower density within $k G$ features they rise to the surface and align themselves vertically with respect to the solar surface (Schüssler 1986). Observations, however, reveal that typical inclinations in the network and plage are around $10^{\circ}-15^{\circ}$ (Martínez Pillet et al. 1997; Sanchez Almeida and Martinez Pillet 1994; Bernasconi et al. 1995), which likely results from buffeting of these features by granular convection, causing them to have a preferred non zero inclination. Both in the network and plage $k G$ features have no preferred azimuthal orientation (Martínez Pillet et al. 1997). Deviations from these values are expected when such a feature resides underneath a sunspot's canopy (Solanki 1993) or short time intervals are considered (Steiner et al. 1996).

Whilst many key properties of small $k G$ features have been uncovered over the last five decades, many questions remain as of yet unresolved. Apart from the overriding question of how photospheric observations connect to chromospheric and coronal observation, reconstructing the full photospheric atmosphere by inverting observed Stokes spectra will be the key stepping stone in recovering the various velocity, magnetic field, inclination as well as other gradients in addition to the general properties already discovered. The means to perform such an investigation have only recently become available in the form of the high quality images of Hinode SOT/SP, both spectrally and spatially, and the recently developed spatially coupled 2D SPINOR inversion code (van Noort 2012). Chapters 3 and 4 will elaborate upon the results obtained via this new inversion technique from SOT/SP images of plage. 



\section{Quiet Sun magnetic fields observed by Hinode: Support for a local dynamo}

\subsection{Abstract}

The Hinode mission has revealed copious amounts of horizontal flux covering the quiet Sun. Local dynamo action has been proposed to explain the presence of this flux. We sought to test whether the quiet Sun flux detected by Hinode is due to a local or the global dynamo by studying long-term variations in the polarisation signals detectable at the disc centre of the quiet Sun between November 2006 and May 2012, with particular emphasis on weak signals in the internetwork. The investigation focusses on line-integrated circular polarisation $V_{\text {tot }}$ and linear polarisation $L P_{\text {tot }}$ profiles obtained from the Fe I $6302.5 \AA$ absorption line in Hinode SOT/SP. Both circular and linear polarisation signals show no overall variation in the fraction of selected pixels from 2006 until 2012. There is also no variation in the magnetic flux in this interval of time. The probability density functions (PDF) of the line-of-sight magnetic flux can be fitted with a power law from $1.17 \times 10^{17}$ Mx to $8.53 \times 10^{18} \mathrm{Mx}$ with index $\alpha=-1.82 \pm 0.02$ in 2007 . The variation of $\alpha$ 's across all years does not exceed a significance of $1 \sigma$. Linearly polarised features are also fitted with a power law, with index $\alpha=-2.60 \pm 0.06$ in 2007. Indices derived from linear polarisation PDFs of other years also show no significant variation. Our results show that the ubiquitous horizontal polarisation on the edges of bright granules seen by Hinode are invariant during the minimum of cycle 23 . This supports the notion that the weak circular and linear polarisation is primarily caused by an independent local dynamo.

\subsection{Introduction}

Quiet Sun observations made by the SOT (Tsuneta et al. 2008b; Suematsu et al. 2008; Ichimoto et al. 2008; Shimizu et al. 2008) on Hinode (Kosugi et al. 2007) as well as IMaX (Martínez Pillet et al. 2011) on SunRise (Solanki et al. 2010; Barthol et al. 2011) have revealed copious amounts of horizontal magnetic flux. These observations, using both Hinode SOT/SP and SOT/NFI, also showed that this horizontal magnetic flux is concentrated in patches on the edges of bright granules (Lites et al. 2008; Ishikawa et al. 2008). Danilovic et al. (2010a) found using SunRISE that the lifetime of these flux patches ranges from one minute right up to the granular lifetime of five minutes. The apparent 
isolation of these flux patches from other quiet Sun phenomena have made them strong candidates for being generated by local dynamo action just below the photosphere (Cattaneo 1999; Danilovic et al. 2010b; Ishikawa and Tsuneta 2009). Also, using SOT/SP data from plage regions and quiet Sun, Ishikawa et al. (2008) showed that these fields have no preferred orientation and are independent of the strength of nearby magnetic fields. The flux distributions of magnetic features obtained from SOHO/MDI and Hinode SOT/NFI have also been interpreted in terms of a turbulent dynamo occurring continuously over a range of scales throughout the convection zone (Parnell et al. 2009; Thornton and Parnell 2011).

In spite of the evidence presented in the papers above, the true source of the weak magnetic flux features in the quiet Sun is still not resolved (Petrovay and Szakaly 1993). In particular it is unclear if they are produced by a turbulent local dynamo (Vögler and Schüssler 2007; Schüssler and Vögler 2008; Danilovic et al. 2010b; Pietarila Graham et al. 2010), with predominantly horizontal magnetic fields (Lites et al. 2008; Orozco Suárez et al. 2007) or due to the global dynamo, e.g. field recycled from decaying active and ephemeral active regions. Asensio Ramos (2009) concludes that quiet Sun magnetic fields observed by Hinode have an isotropic distribution and Stenflo (2010) finds predominantly vertical fields speaking against a local dynamo. Stenflo (2012) argues using SOHO/MDI data that the global dymano is the main source of magnetic flux even in the quiet Sun. Magnetic flux could also be generated from rising magnetic loops that "explode" within the convection zone (Rempel and Schüssler 2001). A combination of different sources is also conceivable.

One way to distinguish between global and local dynamos is to consider the variation of the magnetic flux over the solar cycle, since, put simply, flux due to a local dynamo is not expected to vary over the solar cycle, while flux produced by the global dynamo should display a cyclic variation. There is some uncertainty regarding the magnitude of this variation due to the fact that smaller magnetic bipoles, such as ephemeral active regions, show a rather low cycle amplitude, with shifted phase relative to the strong cyclic variation of active regions (Harvey|1993; Hagenaar 2001; Jin and Wang 2012).

The Hinode satellite has recorded images of the quiet Sun for over six years now, covering the extraordinary solar minimum of cycle 23. Solar activity during this minimum was markedly lower than in the minima of previous solar cycles (Lockwood et al.2010; Fröhlich 2009). Although this period is considerably shorter than a full solar cycle, we nonetheless use all available Hinode SOT/SP quiet Sun disk centre scans over this period to address the question whether solar cycle variations can be measured down to granular scales.

\subsection{Data and method of analysis}

Here we focus entirely on the quiet Sun observed close to the solar disc center from November 2006 to May 2012 and Hinode SOT/SP images were chosen accordingly. In this Section we present the data employed and describe the various issues that presented themselves and how they were accounted for.

Since this investigation is concerned with the weak polarisation signals in the quiet 
Sun, a good Signal-to-Noise ratio is paramount. Therefore, images with an exposure time of at least $4.8 \mathrm{~s}$ operated in dual mode were preferred. However, due to telemetry problems starting in 2008 dual mode images were only available until January 2008. Images recorded after January 2008 were single mode images. To increase the statistical significance and to minimise selection effects, images covering a large area of the solar disc were preferred, ranging up to $320 " \times 160$ ".

Apart from 2007 the number of images satisfying these criteria was rather patchy, so that images recorded as part of the irradiance program were used (HOP 79). Images belonging to HOP 79 were shot almost every month and cover the quiet Sun at the disc center. However, two issues emerged during the investigation while working with these images. Firstly they received an onboard binning in the slit direction, which decreased the resolution and required, for consistency, all the other images used in this investigation to be binned retrospectively. Also, the images in the irradiance program are comparatively small, only 30 " $\times 120$ " each. This was partly compensated by using two images from the irradiance program recorded on the same day, but on different locations around the disc center, thereby increasing the considered area to 30 " $\times 240$ ". Table 2.1 gives an overview of all the images used in this investigation. Every image was calibrated using the $s p \_$prep routine of the solarsoft package, followed by a bitshift correction of the four Stokes parameters that mainly affected images recorded in dual mode.

\subsubsection{Instrumental effects}

The analysis of the calibrated images was performed by a pixel selection algorithm. Pixels were selected according to a preset uniform polarisation threshold. Finding a robust threshold proved to be a delicate process, because images recorded in 2007 in dual mode have a smaller noise level by a factor of $\sqrt{2}$ than single mode images recorded in 2008 . Images from the irradiance program have a different noise level yet again due to the onboard binning and subsequent jpeg compression. This problem was minimised by reducing calibrated dual mode images from 2007 to single mode images and subsequently binning them. After this process was completed every image had the same effective pixel size $(0.16 " \times 0.32$ ") as well as the same, within one significant figure, standard deviation $(\sigma)$ noise level for the Stokes $Q, U$ and $V$ parameters estimated from the continuum pixels. The standard deviation $(\sigma)$ was found to increase by $10 \%$ over six years starting from a value of $\sigma=0.9 \times 10^{-3} I_{c}$ in November 2006 .

The rms contrast of Stokes $I$ was also calculated to discern any variation in the PSF over time. We found that it takes an average value of $7 \pm 0.3 \%$. This is in good agreement with the results of Danilovic et al. (2008), who also also obtained a value of $7 \%$ prior to the deconvolution by the instrument's PSF. However, the continuum contrast, shown in Fig. 2.1, is not constant over the period of investigation. It can vary by as much as $\approx 1 \%$ and is caused by temperature fluctuations on the spacecraft (Katsukawa private communication). This variation also affects the Stokes $Q, U$ and $V$ profiles in an image, analyzed in Sects. 3 and 4 , and therefore needs to be compensated.

Every image was convolved by an appropriate artificial defocus using an MTF generated by the commercial software package ZEMAX following Danilovic et al. (2008). The convolution was applied to all 112 wavelength positions of each Stokes parameter. The result of this convolution on the rms contrast can also be seen in Fig. 2.1 where the 

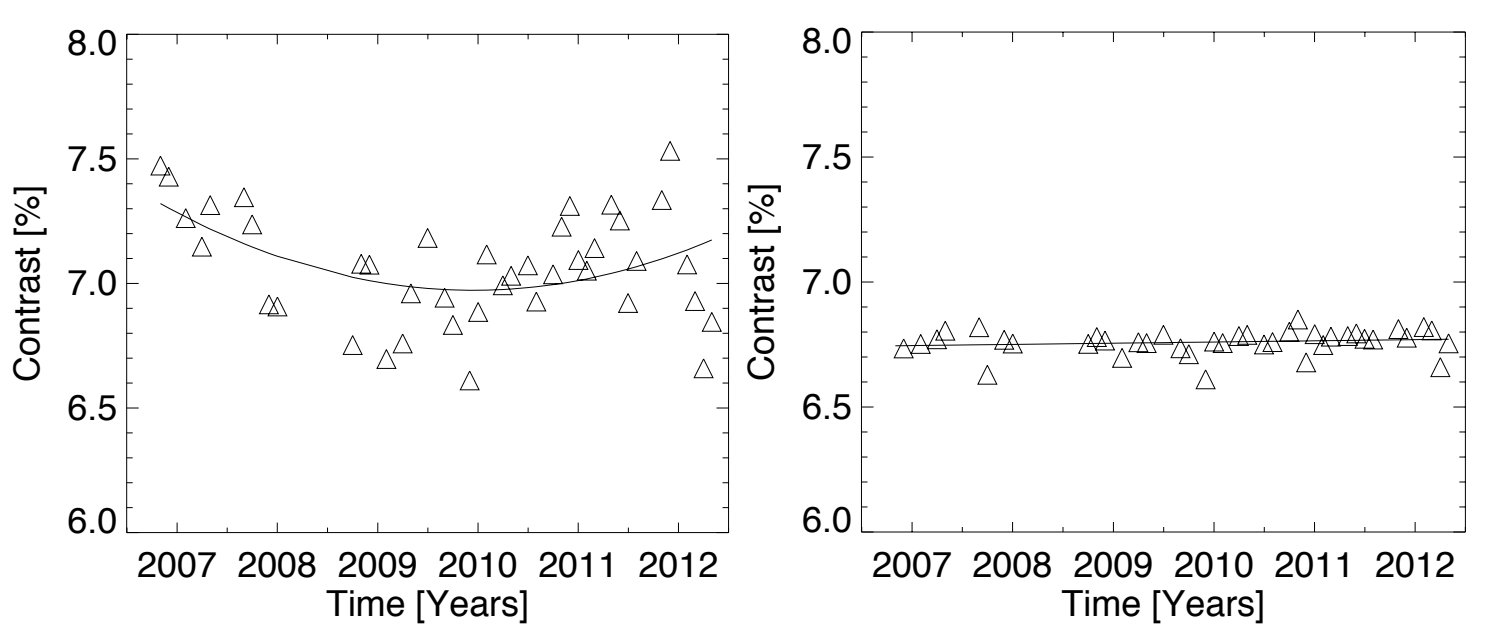

Figure 2.1: Left: Original Stokes I continuum contrast variation of the employed SOT/SP images. The solid line is a quadratic fit with quadratic and linear coefficients of $0.00025 \pm$ 0.00009 and $-0.019 \pm 0.006$. Right: Stokes $I$ continuum contrast variation after each image was convolved using an artificial defocus. The solid line is a constant drawn at $6.75 \%$.

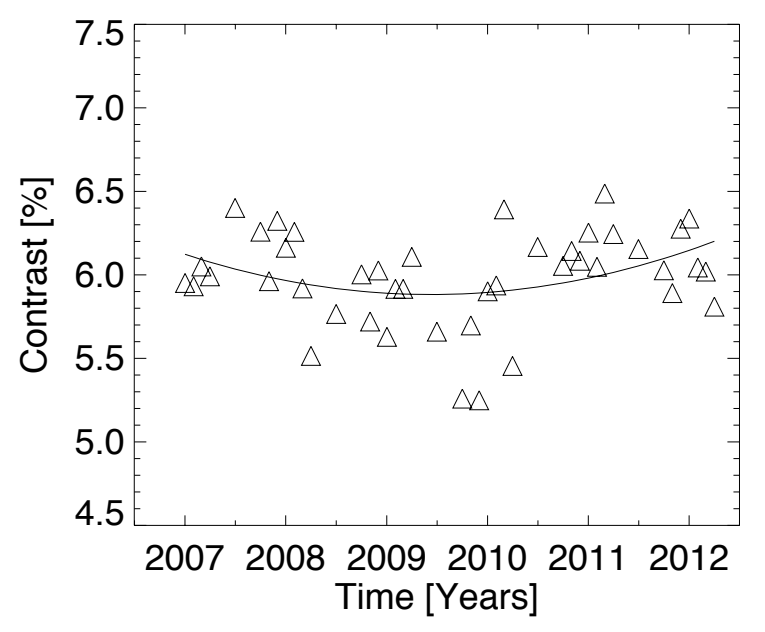

Figure 2.2: Contrast variation of SOT/BFI red continuum images at $6684 \AA$. The solid line is a quadratic fit with quadratic and linear coefficients of $0.00028 \pm 0.00013$ and $-0.017 \pm 0.009$.

remaining scatter is the result of the applied artificial defocus being in steps of $0.17 \mathrm{~mm}$ similar to the defocus steps available on the Hinode spacecraft.

The variation of rms contrast not only affects SOT/SP but has also been observed with the filtergraph aboard Hinode. Muller et al. (2011) found a reduction in the continuum contrast using images from SOT/BFI between November 2006 until July 2010. By using wide band continuum images recorded at $6684 \AA$ we not only found a decrease in the continuum contrast until July 2010, but a subsequent increase until Jan 2012 amounting to a variation of $\approx 1 \%$. Unlike the spectrograph, the filtergraph has regular focus campaigns to ensure that the instrument is in the optimum focus position. Since both instruments show the same variation within error this rules out the possibility that the contrast variation seen in Fig. 2.1 is merely the result of a relative shift between the filtergraph and the spectrograph. 


\subsubsection{Image analysis}

In order to avoid any biases introduced by inversions see (Borrero and Kobel 2011), we analysed the observed Stokes profiles directly. In parts of this investigation an estimate of the magnetic flux density was made, based on the observed line-of-sight polarisation signals. This was done using the standard magnetograph formula (Unno 1956). However, this formula suffers from the assumption of a weak magnetic field, which is not fulfilled by network magnetic elements and not even by all magnetic features in the internetwork (Lagg et al. 2010). This formula also assumes certain atmospheric parameters to be constant, such as the line-of-sight velocity and the magnetic field strength, which is not true for weak quiet Sun Stokes profiles (Viticchié and Sánchez Almeida 2011). Therefore the magnetic flux density value returned by the formula must be considered as an approximation only. In addition, this analysis assumes that the magnetic feature is resolved, so that the flux density is underestimated if this assumption is not fulfilled. The estimate of magnetic flux of the feature should not be affected, however.

Several steps were taken to improve the Signal-to-Noise ratio and thus increase the number of pixels above a given threshold. The entire investigation focussed only on the Fe I $6302.5 \AA$ absorption line as it is a Zeeman triplet and has a larger Landé factor $(g=$ 2.5) compared to the Fe I $6301.5 \AA$ absorption line. It, therefore, is more sensitive to weak magnetic fields. The total circular polarisation signal was calculated using $M=9$ wavelength points around the central wavelength of the Fe I $6302.5 \AA$ absorption line.

$$
V_{\text {tot }}=\frac{1}{M} \sum_{1}^{M}|V| .
$$

The absolute value of the Stokes $V$ signal was taken at each wavelength point to account for the full polarimetric signal. The fraction of pixels displaying significant circular polarisation, $P_{s e l}\left(V_{t o t}\right)$, in an image could then be calculated. The average non-zero $r m s$ noise of these profiles was removed from $V_{\text {tot }}$ before being compared to a threshold. The magnitude of the offset was calculated for each profile individually using wavelength points located in the continuum of the spectrum.

Instead of using the individual Stokes $Q$ and $U$ profiles, the total linear polarisation, $L P$, was calculated and is defined as,

$$
L P=\frac{\sqrt{Q^{2}+U^{2}}}{I_{c}} .
$$

Although increasing the signal-to-noise ratio, this process meant that any information on the azimuth of the magnetic vector contained in the polarisation signal was lost. Finally, the $L P$ profile of the Fe I $6302.5 \AA$ line was integrated along the dispersion direction using,

$$
L P_{t o t}=\frac{1}{M} \sum_{1}^{M} L P .
$$

The mean non-zero rms noise that arises in the calculation of $L P_{\text {tot }}$ was removed for each of the $M=9$ wavelength points before a constant linear polarisation threshold for $L P_{t o t}$ was set. Then $P_{\text {sel }}\left(L P_{t o t}\right)$, the fraction of pixels selected on the basis of the above criteria, 


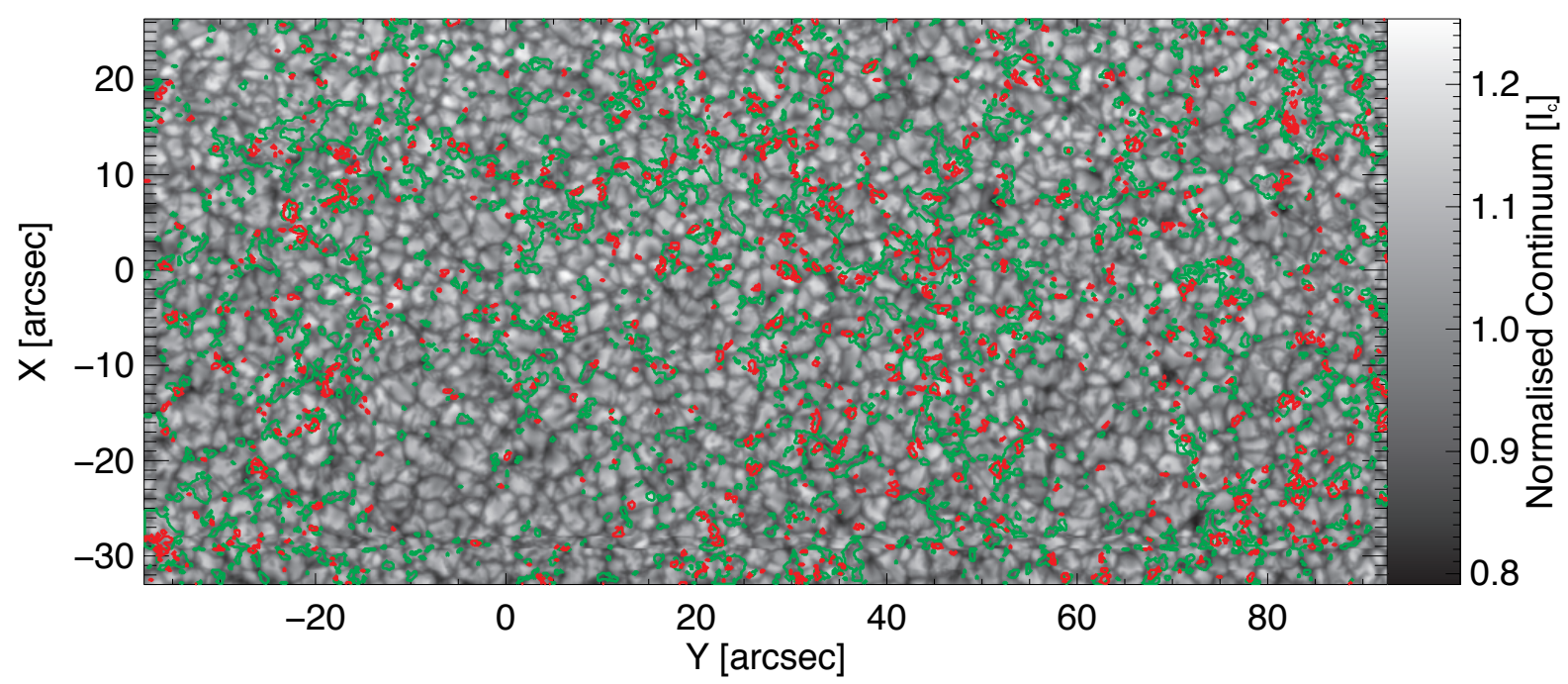

Figure 2.3: Quiet Sun continuum image from $30^{\text {th }}$ January 2008 UT 23:34:05 with overplotted contours of both circular and linear polarisation patches corresponding to $\approx 7 \sigma$ for circular polarisation (green) and $\approx 3 \sigma$ for linear polarisation (red). The image was subjected to an $2 \times 1$ binning in the slit direction. Each contour encompasses a patch of at least two pixels in size.

could be determined.

The fraction of pixels lying above this threshold is called the occurrence of the circular, $P_{s e l}\left(V_{t o t}\right)$, and of the linear, $P_{s e l}\left(L P_{t o t}\right)$, polarisation. Patches of linear polarisation were analysed separately from those of circular polarisation. One reason for doing so was due to the different locations on an arbitrary quiet Sun image where patches of different polarisation appeared. Patches of strong circular polarsiation were almost exclusively found in the intergranular lanes, whereas patches of linear polarisation preferred the edges of granules (Lites et al. 2008; Ishikawa et al.2008). Figure 2.3 is a typical quiet Sun image used in this investigation with the contours showing regions with significant polarisation signals (see caption for details).

The results presented in Sections $3 \& 4$ were obtained by setting a threshold of $V_{\text {tot }}=0.3 \%(\approx 7 \sigma)$ on the circular polarisation and $L P_{t o t}=0.095 \%(\approx 3 \sigma)$ on the linear polarisation, respectively. The same threshold was chosen for all images. Isolated pixels that met a particular polarisation threshold were discarded, to prevent the selection of pixels caused by noise spikes. This precaution decreased the number of selected pixels in an image by $15 \%-20 \%$, so that on average $11 \%$ of pixels in an image carried significant circular polarisation and 3\% significant linear polarisation. The exact values of the chosen thresholds do not change the results significantly, as we learnt by trying out different values. However, raising the value of the threshold for $L P_{t o t}$ led to a rapid decrease in the number of chosen pixels and thus to significantly poorer statistics, while for $V_{t o t}$ this is less of an issue. However, small thresholds for $V_{\text {tot }}$ compromised the patch size analysis presented in the following sections by merging the many of the strong flux concentrations in an image. Since our conclusions are not affected, descriptions of analyses employing higher or lower 


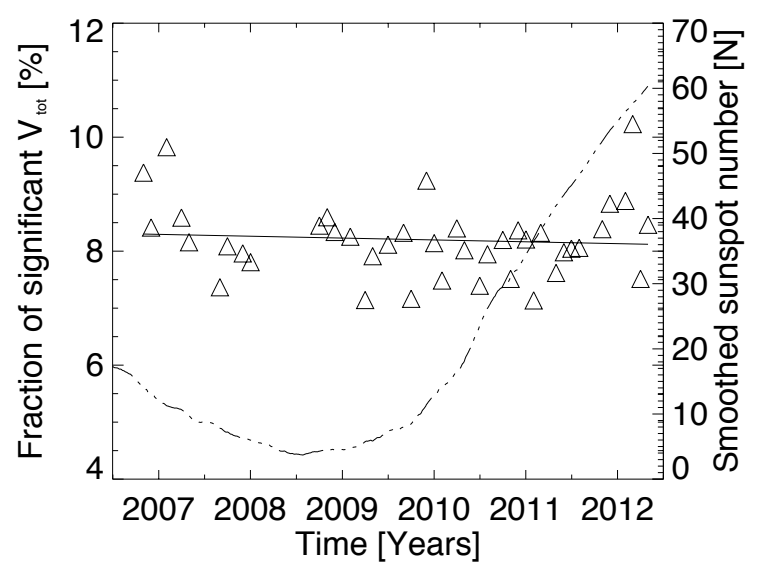

Figure 2.4: Fraction of pixels containing circular polarisation above a threshold of $\approx 7 \sigma$, $P_{\text {sel }}\left(V_{t o t}\right)$, from November 2006 until May 2012 from the original unconvolved images. The solid line is a regression with slope $-0.002 \pm 0.005$.

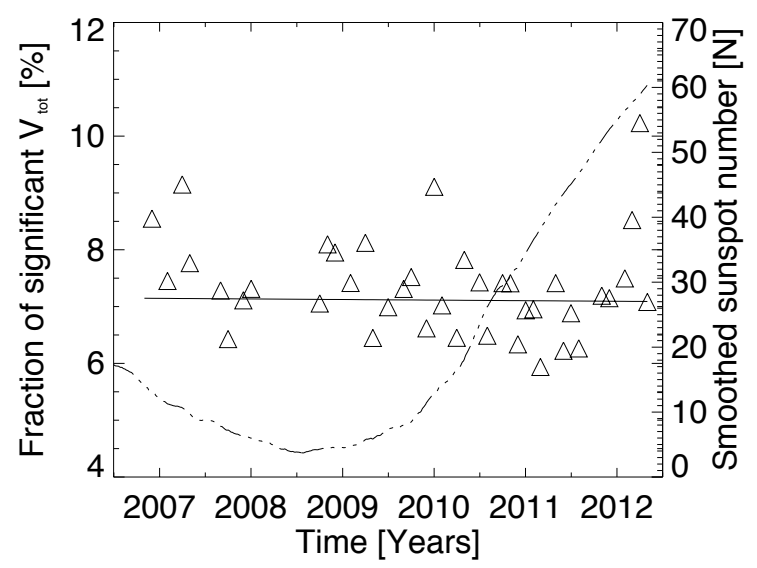

Figure 2.5: $P_{s e l}\left(V_{t o t}\right)$ after each image was convolved with an appropriate artificial defocus. The solid line is a regression with slope $-0.001 \pm 0.007$.

thresholds have been omitted.

\subsection{Results: circular polarisation}

As outlined in Sect. 2 individual pixels in an image were selected on the strength of their polarisation signal $V_{t o t}$ and $L P_{t o t}$. This value was calculated according to Eqs. 1 and 3 and subsequently compared to a uniform threshold that was the same for all images. The $V_{t o t}$ threshold of $0.3 \%$ corresponded to a polarisation signal of $\approx 7 \sigma$.

First the occurrence, $P_{s e l}\left(V_{t o t}\right)$, of the circular polarisation was investigated both, before and after the convolution of the original images with an artificial defocus. Fig. 2.4 shows the variation of $P_{\text {sel }}\left(V_{t o t}\right)$ from the original images i.e. before the defocus correction. The regression in this plot has a slope of $-0.002 \pm 0.005$ [\%/month]. and indicates that there has been no significant change in the occurrence of Stokes $V$ signals. Fig. 2.5 shows the 


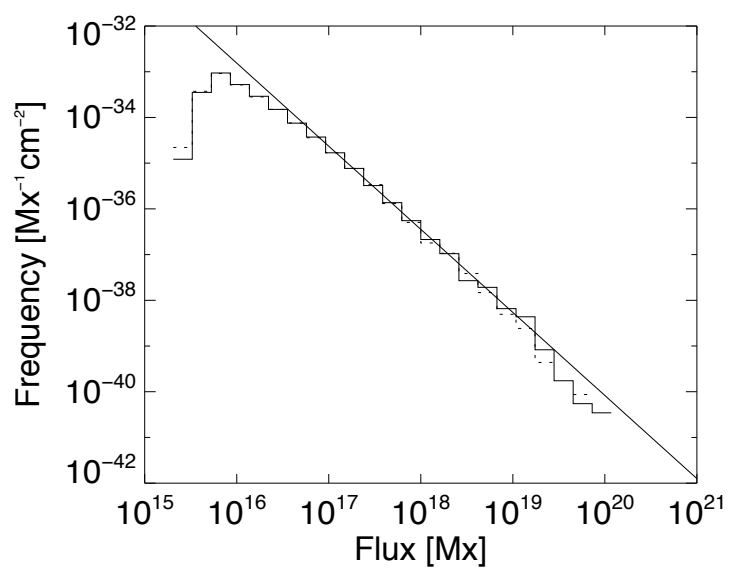

Figure 2.6: Two distributions of the line-of-sight magnetic flux, $\mathrm{Mx}$, found on the quiet Sun. The solid line refers to a distribution from 2007, whereas the dotted line refers to 2010. The regression shows a power law fit to the 2007 distribution with spectral index $\alpha=-1.82 \pm 0.02$.

occurrence of Stokes $V$ signals at the same $0.3 \%$ threshold after each image was convolved with an artificial defocus. As expected, the convolution has decreased the total number of selected pixels per image, but still no significant trend is discernible as the fitted regression has a slope of $-0.001 \pm 0.007[\% /$ month $]$.

There is a noticeable scatter between individual images in both Figs. 2.4 and 2.5. Even with the largest images included in this study the number of large network elements varied considerably from image to image. The sudden increase in $P_{\text {sel }}\left(V_{t o t}\right)$ from images recorded in 2012, when compared to 2010 or 2011 , could be the result of magnetic flux from decaying active regions which are found ever closer to the disc centre as cycle 24 progresses. A quadratic fit to Figs. 2.4 and 2.5 both show a quadratic coefficient of $0.0008 \pm 0.0003$. However, the large variation in the number of network elements question the reliability of these fits.

Possible year-to-year variations in $P_{\text {sel }}\left(V_{t o t}\right)$ that may be due to the solar cycle were investigated further with the help of probability density functions (PDFs) for which $V_{t o t}$ was converted to line-of-sight magnetic flux, Mx, using the magnetograph formula. The flux values obtained by this formula were compared to those calculated by a Milne-Eddington inversion for a single image recorded in 2007 and were found to be compatible with each other. Distributions corresponding to each year of the investigation were produced by combining all the individual images from each year. By comparing such PDFs we can check if features with different amounts of flux all behave similarly or if e.g. larger flux features behave differently.

Two PDFs are displayed in Fig. 2.6 on logarithmic scales. The magnetic flux values shown on the $x$-axis correspond to flux per feature. The magnetic flux of a feature was determined by the sum of the magnetic flux found in the number of pixels that were directly connected to each other. A power law was fitted to each distribution using a least squares minimisation technique. The power law takes the form, $n(\Phi)=n_{0}\left(\Phi / \Phi_{0}\right)^{\alpha}$. The PDF for 2007, solid line, has a spectral index of $\alpha=-1.82 \pm 0.02$, with $n_{0}=1.68 \times 10^{-35} \mathrm{Mx}^{-1} \mathrm{~cm}^{-2}$ 


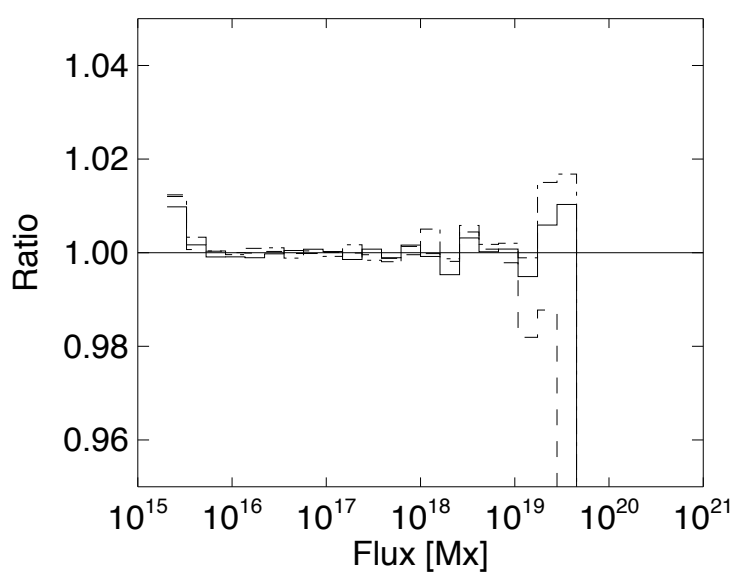

Figure 2.7: Ratio of three line-of-sight magnetic flux distributions, 2009: solid, 2011: dashed, and 2012: dot-dashed with respect to 2007 using Eq. 4. A solid $y=1$ line has been drawn for reference.

and $\Phi_{0}=1.17 \times 10^{17} \mathrm{Mx}$, shown by the regression in Fig. 2.6. The PDF for 2010, dotted line, has a spectral index of $\alpha=-1.84 \pm 0.03$, where $n_{0}$ and $\Phi_{0}$ are the same as in 2007 up to four decimal places. Both distributions have the same binning and were fitted over the range from $n_{0}$ up to $8.53 \times 10^{18} \mathrm{Mx}$. Larger fluxes were not fitted as the number of features carrying them was very small in each year. The uncertainty for each spectral index was calculated by taking into account the uncertainty associated with every bin in the distribution over the fitted range of fluxes. By varying the value of $n_{0}$ for any distribution the spectral index for that distribution was seen to vary from -1.73 to -1.90 . However, as long as the binning and fitted range were equal for each distribution, the variations between the yearly power law indices had a significance of only $1 \sigma$ or less. The indices for the two distributions shown in Fig 2.6 agree well with the indices calculated by Parnell et al. (2009) and Iida et al. (2012) using SOT/NFI, despite the differing feature selection algorithms and magnetic flux calculation. The similarity between the yearly PDFs can be demonstrated further with the help of a ratio between these distributions. The ratio between two distributions was calculated using,

$$
R=\frac{\frac{1}{K} \sum_{i}^{K} f_{i}^{20 x x}}{\frac{1}{N} \sum_{i}^{N} f_{i}^{2007}},
$$

where $f$ is the particular PDF of an image and both $K$ and $N$ correspond to the number of images used in the PDFs for 20xx and 2007 respectively. The distribution corresponding to 2007 was used as a 'benchmark' against which other distributions were compared, as it was composed of the largest images and hence enjoyed the best statistics. The ratios from the distributions of the years 2009, 2011 and 2012 are shown in Fig. 2.7 and indicate that up to fluxes of $1 \times 10^{19} \mathrm{Mx}$ the differences between the distributions are less than $1 \%$, only larger fluxes show differences $>1 \%$. However, due to the small number of these large-flux features the differences bear no statistical significance. Nonetheless, differences seen for fluxes $>1 \times 10^{19} \mathrm{Mx}$ offer an explanation for the scatter observed in Figs. 2.4 and 2.5. since the addition or removal of only one such feature causes a large effect in $P_{\text {sel }}\left(V_{t o t}\right)$ as it is composed of a large number of pixels. Ratios involving 2008 and 2010 give essentially 


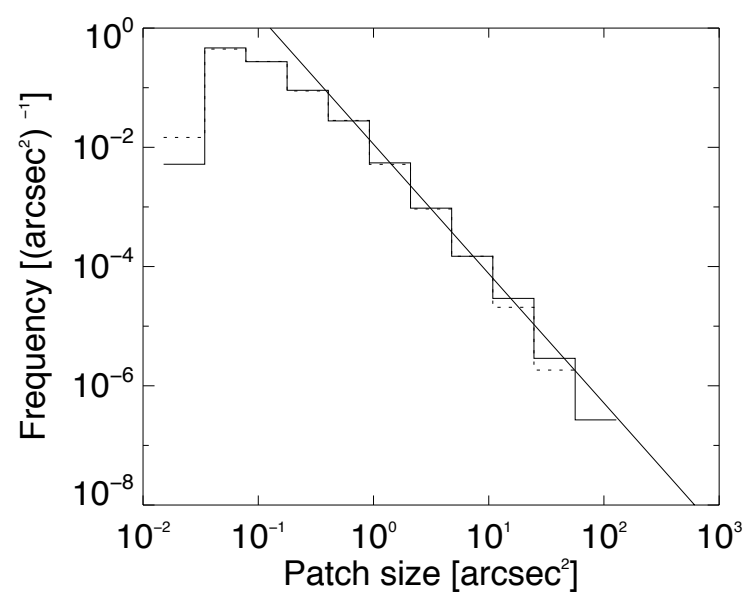

Figure 2.8: Two distributions of circular polarisation patch sizes. The solid line refers to a distribution from 2007, whereas the dotted line corresponds to 2010. The regression is a power law fit to the 2007 distribution with spectral index $\alpha=-2.20 \pm 0.05$.

the same result.

This can be illustrated further by generating yearly distributions of patch sizes present on the quiet Sun. The two distributions shown in Fig. 2.8 on logarithmic scales correspond to 2007 and 2010. The distribution for 2007, solid line, has been fitted using a power law with spectral index $\alpha=-2.17 \pm 0.04$. The 2010 distribution, dotted line, is also shown in Fig. 2.8 with spectral index $\alpha=-2.20 \pm 0.05$. The power laws in Fig. 2.8 were fitted over the range from $1.39 \operatorname{arcsec}^{2}$ to $37.28 \operatorname{arcsec}^{2}$. As was the case with the magnetic flux PDFs, the yearly variations between the patch size PDFs is smaller than $1 \sigma$ as long as the range of the fit and the binning is the same for every distribution. The similarity between the distributions is confirmed further by taking the ratio between them using Eq. 4. The three ratios shown in Fig. 2.9 used distributions from the same years as in Fig. 2.7. It supports the conclusion drawn from Fig 2.7 that larger patches in particular those $>1 \operatorname{arcsec}^{2}$ are mainly responsible for the scatter seen in Figs. 2.4 and 2.5. Also, the distribution of areas drops much more rapidly than the area-integrated line-of-sight flux, illustrating that the line-of-sight flux averaged over the patch increases quickly with patch size.

\subsection{Results: linear polarisation}

The linear polarisation was investigated along the same lines as the circular polarisation in section 3. Individual pixels in an image were selected according to the strength of their average total linear polarisation signal $L P_{t o t}$, calculated as described in Sect. 2. As was the case with the circular polarisation a constant uniform threshold was set for all images against which individual values of $L P_{\text {tot }}$ in each image were compared. The common threshold used for the linear polarisation was $0.095 \%$, which corresponds to a polarisation signal at $\approx 3 \sigma$. The images were also analysed using higher thresholds for $L P_{t o t}$, but the results presented in this section showed no significant dependence on the choice of $L P_{t o t}$. Higher thresholds severely reduced the number of selected pixels, since the number of 


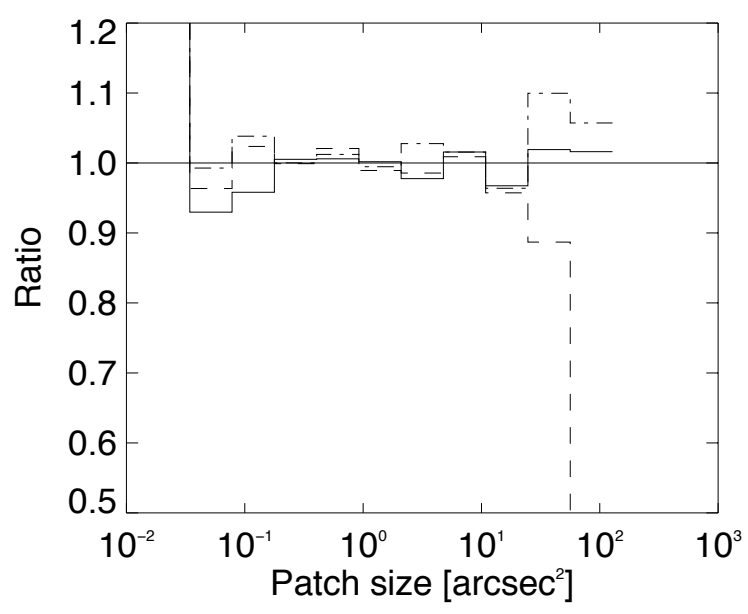

Figure 2.9: Ratio of three circular polarisation patch size distributions, 2009: solid, 2011: dashed, and 2012: dot-dashed with respect to 2007 using Eq. 4. A solid $y=1$ line has been drawn for reference.

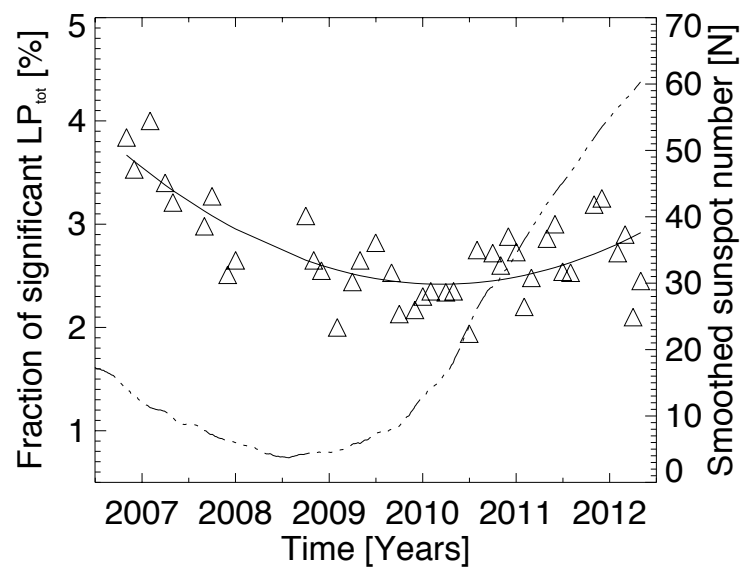

Figure 2.10: Fraction of pixels containing linear polarisation, $P_{\text {sel }}\left(L P_{t o t}\right)$, above a threshold of $\approx 3 \sigma$ from November 2006 until May 2012 from the original images. The solid line is a quadratic fit with quadratic and linear coefficients of $0.0008 \pm 0.0001$ and $-0.06 \pm 0.01$.

pixels drops off very rapidly with increasing $L P_{t o t}$ signal, much more rapidly than for $V_{t o t}$.

Therefore results for a $L P_{t o t}$ threshold other than $0.095 \%$ are not discussed further in this section. First the variation in the occurrence, $P_{\text {sel }}\left(L P_{t o t}\right)$, with respect to time was investigated and is shown in Figs. 2.10 and 2.11 .

The $P_{\text {sel }}\left(L P_{t o t}\right)$ in the original unconvolved images is plotted in Fig. 2.10. It shows that $P_{\text {sel }}\left(L P_{t o t}\right)$ dropped by $\approx 30 \%$ from November 2006 until the beginning of 2010 followed by an increase until May 2012. The quadratic coefficient of the fit in this plot has a value of $0.0008 \pm 0.0001$, i.e. significant at the $8 \sigma$ level. The minimum of $P_{\text {sel }}\left(L P_{t o t}\right)$ took place during the beginning of 2010, which is one and half years after the official minimum (December 2008) seen in the Sunspot number. Fig. 2.11 shows the $P_{\text {sel }}\left(L P_{\text {tot }}\right)$ after each image has been convolved. The convolution has completely removed the quadratic term present in Fig. 2.10, demonstrating how thoroughly the contrast variation affects the 


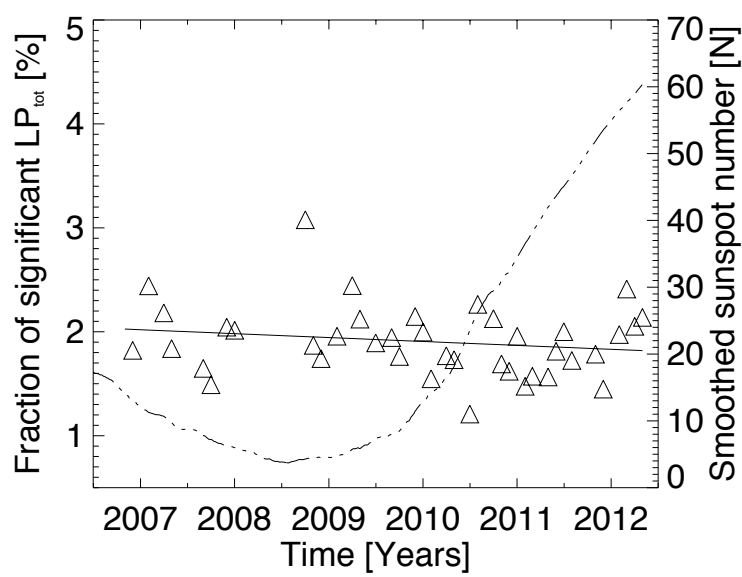

Figure 2.11: Fraction of pixels containing linear polarisation, $P_{s e l}\left(L P_{t o t}\right)$, above a threshold of $\approx 3 \sigma$ after each images was convolved with an appropriate artificial defocus. The solid line is a regression with a slope of $-0.003 \pm 0.003$. The triple-dot-dash line shows the smoothed sunspot number over the same period.

observed polarisation signal. The solid regression line in Fig 2.11 has a slope of -0.003 \pm 0.003 and suggests that $P_{\text {sel }}\left(L P_{\text {tot }}\right)$ has remained invariant throughout the period of investigation. However, as was the case with the circular polarisation in Figs. 2.4 and 2.5 the average $P_{\text {sel }}\left(L P_{\text {tot }}\right)$ in 2010 and 2011 is lower than in 2007.

The decrease in $P_{\text {sel }}\left(L P_{t o t}\right)$, suggested in Fig. 2.11, was investigated further by comparing yearly PDFs of linear polarisation features. The linear polarisation of a feature was defined as the sum of the linear polarisation signal of pixels that were directly connected to each other. The distribution obtained from the data gathered in the years 2007, solid line, and 2010, dotted line, are shown in Fig. 2.12. Again a power law of the form $n(L P)=n_{0}\left(L P / L P_{0}\right)^{\alpha}$ was fitted to each distribution using a least squares minimisation technique. The power laws in Fig. 2.12 were fitted over the range $0.018 \mathrm{LP} P_{\text {tot }}$ to $0.207 \mathrm{LP}$ tot , where $L P_{0}=0.018 L P_{t o t}$ and $n_{0}=7.83 \times 10^{-17} I_{c}^{-1} \mathrm{~cm}^{-2}$ for the 2007 distribution and $n_{0}=$ $6.51 \times 10^{-17} I_{c}^{-1} \mathrm{~cm}^{-2}$ for the 2010 distribution. The spectral indices are $\alpha=-2.60 \pm 0.06$ for 2007 and $\alpha=-2.62 \pm 0.09$ for 2010. The uncertainties for each spectral index took into account the uncertainties of each bin over the fitted range. Again the yearly variations of the power law indices are within $1 \sigma$ as long as the binning and the fitted range is the same for each distribution. By varying $n_{0}$ the spectral index of a given distribution was seen to vary from -2.34 to -2.87 . Even though the spectral indices of the two distributions in Fig. 2.12 do not differ significantly from each other, a closer inspection shows that the 2010 distribution appears to be consistently lower in frequency across all bins. This can be demonstrated more clearly by taking a ratio between the yearly distributions using Eq. 4 . The ratio between the 2011 and 2007 distributions plotted in Fig. 2.13, dashed line, shows that linear polarisations features with $L P_{\text {tot }}>0.0016 I_{c}$ are consistently less frequent in 2011 than in 2007. However, at no point does this difference lie above a $1 \sigma$ significance, which corresponds to $\approx 4 \%$ over the range $1 \times 10^{-3}-1 \times 10^{-1} I_{c}$. The 2010 distribution shows a similar ratio. All other ratios are centered around unity as is illustrated by the two other ratios plotted in Fig. 2.13, where the solid line shows 2009 vs 2007 and the dot-dashed line corresponds to 2012 vs 2007 ratio. 


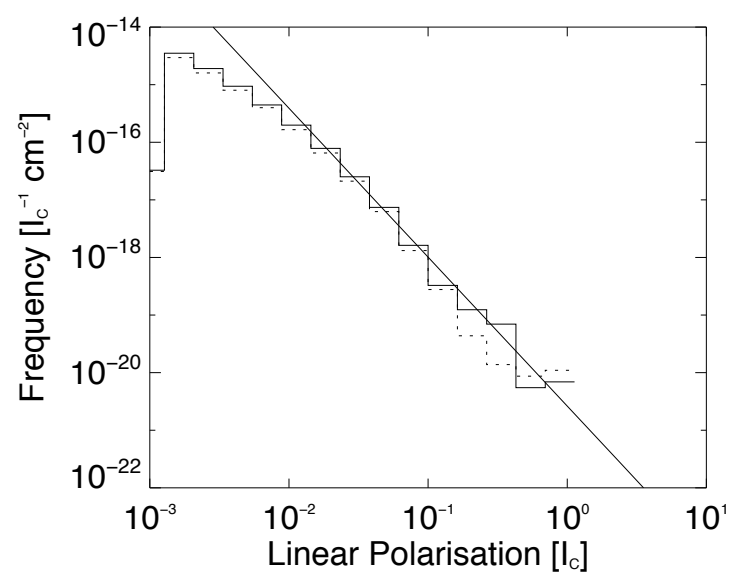

Figure 2.12: Distributions of the linear polarisation, $I_{c}$, found on the quiet Sun. The solid line refers to the distribution from 2007, whereas the dotted line refers to 2010. The regression shows a power law fit to the 2007 distribution with spectral index $\alpha=$ $-2.60 \pm 0.06$.

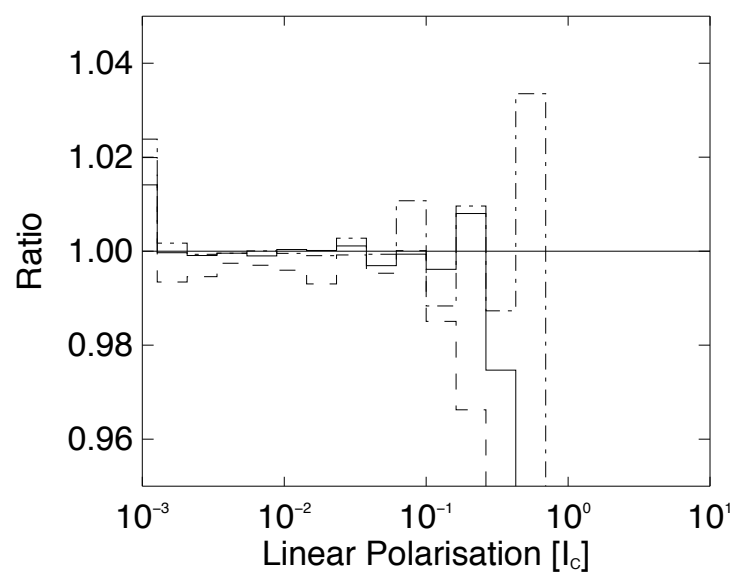

Figure 2.13: Ratio of three linear polarisation distributions, 2009: solid, 2011: dashed, and 2012: dot-dashed with respect to 2007 using Eq. 4. A solid $y=1$ line has been drawn for reference.

Finally, the yearly distributions of the linear polarisation patch sizes were examined and each distribution was fitted using a power law. The 2007 distribution, solid line, and 2010 distribution, dotted line are shown in Fig. 2.14 on logarithmic scales. The spectral indices are $\alpha=-3.03 \pm 0.08$ and $\alpha=-3.19 \pm 0.12$ for 2007 and 2010 respectively. The power laws were fitted over the range from $0.61 \operatorname{arcsec}^{2}$ to $3.16 \operatorname{arcsec}^{2}$. As was the case in Fig 2.12 linear polarisation patches in 2010 are less abundant than in 2007. This is also evident when comparing the ratio between distributions using Eq. 4 shown in Fig 2.15. The three ratios correspond to the same years as for Fig 2.13. The 2011 vs 2007 ratio, dashed line in Fig 2.15 is below unity for all patch sizes greater than $0.052 \operatorname{arcsec}^{2}$, the same is observed for 2010 vs 2007 ratio, not shown here. The difference has a significance of $<1 \sigma$ for patches $>0.268 \operatorname{arcsec}^{2}$ but for smaller patches a significance of $>3 \sigma$ is recorded. The indices of the patch size power laws for Figs. 2.8 and 2.14 cannot be compared directly 


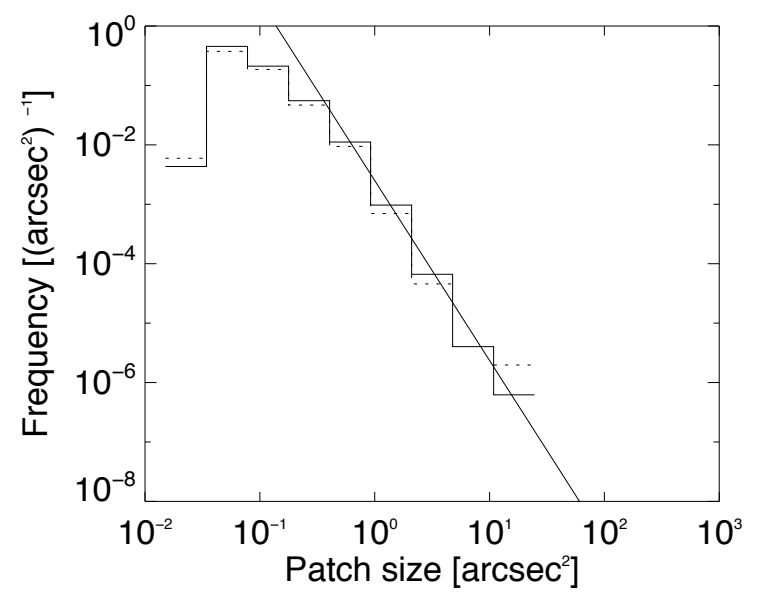

Figure 2.14: Two distributions of linear polarisation patch sizes. The solid line refers to a distribution from 2007, whereas the dotted line corresponds to 2010. The regression is a power law fit to the 2007 distribution with spectral index $\alpha=-3.03 \pm 0.08$.

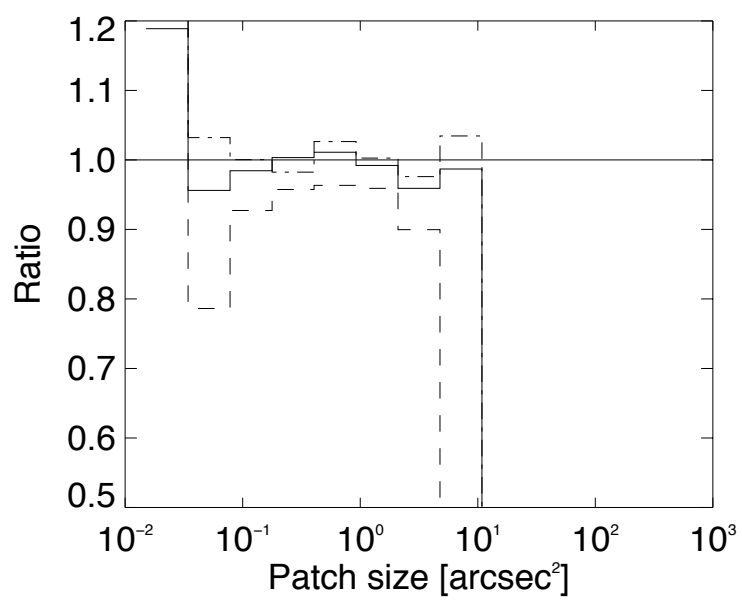

Figure 2.15: Ratio of three linear polarisation patch size distributions, 2009: solid, 2011: dashed, and 2012: dot-dashed with respect to 2007 using Eq. 4. A solid horizontal line indicating a unit ratio has been drawn for reference.

and should be interepreted with care as the power law index of such a distribution can be altered merely by a change in the polarisation thresholds for $V_{t o t}$ and $L P_{t o t}$. Again, the distribution of areas drops much more rapidly than the area-integrated $L P_{t o t}$, indicating that $L P_{t o t}$ averaged over the patch increases rapidly with patch size.

\subsection{Discussion and conclusion}

This investigation sought to clarify whether the number of weak magnetic elements found in the quiet Sun varies with the global solar cycle. To this end 72 Hinode SOT/SP scans of the quiet Sun covering a period from November 2006 until May 2012 were analysed. Patches of circular and linear polarisation were analysed in a similar fashion but were treated separately. Thus, the fraction of pixels with significant polarisation signals was 
considered vs. time and PDFs at different epochs of the spatially averaged longitudinal or transverse field component were compared, as were PDFs of longitudinal or transverse patch sizes.

We discovered that the fraction of pixels with circular polarisation, $P_{\text {sel }}\left(V_{t o t}\right)$, above the threshold remained constant overall during the period of investigation. In particular, features with a line-of-sight magnetic flux $<1 \times 10^{19} \mathrm{Mx}$ showed little variation over time, indicating that magnetic flux found in these features are predominantly governed by a process that is independent of the global solar cycle. This process could take the form of an independent local dynamo action operating close to the solar surface of at least the smaller flux features (Ishikawa and Tsuneta 2009; Pietarila Graham et al. 2009, 2010, Danilovic et al. 2010b; Lites 2011). However, there are also other mechanisms capable of producing photospheric Zeeman signals without requiring flux emergence, such as $U$ loops (Pietarila et al.2011) or the recycling of flux from decaying active regions and network, i.e. flux produced by the global dynamo e.g. (Ploner et al. 2001). The fact that we find no variation over time for this range of magnetic flux is supported by results obtained by Ito et al. (2010) and Shiota et al. (2012) also using Hinode who see the internetwork in the solar polar regions to be invariant with respect to the solar cycle. A study by Kleint et al. (2010) using $C_{2}$ molecular-line Hanle-effect data recorded since the solar maximum of cycle 23 found some evidence to the contrary suggesting that the weak internetwork flux may have a solar cycle dependence.

Possibly the good agreement of our results with those of Ito et al. (2010) and Shiota et al. (2012) are coincidental due to the small field of view of most of the images employed, particularly those from 2008 onwards. The majority of the images available after 2008 cover only an area corresponding to 2-3 supergranules in extend and there may be the possibility that the internetwork magnetic field could vary considerably between supergranules. However, this appears to be of only minor importance within the frame of this investigation. Since the images employed before 2008 cover a much larger area on the solar disk, those large images should provide a better representation of the average quiet Sun. If the variation in the internetwork magnetic fields changes strongly between supergranules then the scatter in Figs. 2.4, 2.5 and particulary 2.10, 2.11 should change measurably from before 2008 to after 2008. However, as the scatter in Figs. $2.5 \& 2.11$ appears to be constant over the whole period of investigation, it can be assumed that even the small images available after 2008 are a reliable representation of the average quiet Sun. Considering that Shiota et al. (2012) not only used images covering the polar regions of the Sun but also employed images recorded at the east limb, near the solar equator, lends further support to the idea that the agreement between their and our results is not coincidental in nature.

Features with a magnetic flux $>1 \times 10^{19} \mathrm{Mx}$ show more variation over time and it is the variation of these features that are mainly responsible for the scatter observed in $P_{\text {sel }}\left(V_{t o t}\right)$ in Figs. 2.4 and 2.5. An analysis of the patch size of these features revealed that they occupy an area of $>2 \operatorname{arcsec}^{2}$. Whilst patches $>2 \operatorname{arcsec}^{2}$ appear to be less abundant in 2010 and 2011 when compared to 2007, these variations are only significant at the $1 \sigma$ level and therefore cannot be used as evidence for a solar cycle dependence of our observed magnetic flux. Nonetheless a significant variation in the number of these features may well be possible and has been suggested by various authors (Harvey 1993; Hagenaar 2001; Jin and Wang 2012). A time series extending more towards activity maximum may be 
required to see such variations. Furthermore, the distribution of the magnetic flux of these features can be fitted using a power law over the range from $1.17 \times 10^{17} \mathrm{Mx}$ to $8.53 \times 10^{18}$ Mx with spectral index, in 2007, of $\alpha=-1.82 \pm 0.02$. This results agrees well with the results of Parnell et al. (2009) and Iida et al. (2012). The index of the power law fit does not vary in time beyond a $1 \sigma$ significance, at least for these quiet Sun features. The long minimum of cycle 23 and the weak cycle 24 appear to be the culprit responsible for the small variation in features with a line-of-sight flux $>1 \times 10^{19} \mathrm{Mx}$ seen in our investigation. To what extend this unusually low activity may also have an impact on the internetwork fields, and hence on our result, is not currently clear. Note, however, that the low activity and extended length of this minimum are not unusual when considering longer term solar activity (e.g., Solanki and Krivova 2011), so that our results may be applicable to typical minima at times of intermediate solar activity.

The fraction of pixels containing significant linear polarisation, $P_{\text {sel }}\left(L P_{t o t}\right)$, supports the observation made using circular polarisation. There is no overall variation in $P_{\text {sel }}\left(L P_{t o t}\right)$ over the investigated time period. This seems intuitive, since the small photospheric loops that dominate the Stokes $Q$ and $U$ signals must have corresponding foot points detectable in Stokes $V$ (Danilovic et al.2010a). Therefore a potential reduction in $L P_{\text {tot }}$ should encompass a response in the circular polarisation as well. The distribution of linear polarisation features can also be fitted with a power law with spectral index, e.g. in 2007 of $\alpha=-2.60 \pm 0.06$. As was the case with the line of sight flux the power law index of the linear polarisation features does not vary in time beyond a significance of $1 \sigma$. The distributions of linear polarisation features for the years 2010 and 2011 show that, for the range $1 \times 10^{-1}>L P_{t o t}>1 \times 10^{-3}$, the frequency of features is consistently lower by $0.5 \%$ when compared to 2007. This difference, however, was found to be insignificant for all bins even when the bins in the distributions were increased.

The patch size distributions of the linear polarisation also showed a drop in frequency for the years 2010 and 2011 when compared to 2007, in particular for patch sizes around 0.052 $\operatorname{arcsec}^{2}$ there was a drop of $20 \%$ at a significance of well beyond $5 \sigma$. What is surprising, is that for the line-of-sight magnetic flux the patch size distributions show no variation when compared to 2007. Also, the drop in the number of patches appears to have no significant effect on the PDF of the linear polarisation features shown in Fig. 2.12.

It needs to be said that the results obtained in this investigation strongly depend on the convolution performed on the images prior to their analysis. As was demonstrated in Figs. 2.5 and 2.11 the effect of a variation in the continuum contrast on the observed polarisation signals is considerable. Indeed, should part of the quadratic behavior of the continuum contrast shown in Fig. 2.1 be solar in origin and not caused by the temperature variations aboard the spacecraft, the internetwork magnetic flux would inevitably show a similar behavior. A certain amount of random continuum contrast variation is almost certainly real, given that a variation in the network and internetwork should influence the number of photospheric bright points (Muller and Roudier 1984; Riethmüller et al.2010).

Future work will focus on monitoring the weak quiet Sun magnetic flux over longer time periods in order to determine if the distribution of the quiet Sun magnetic flux continues to be invariant or whether the weak fields in the internetwork are affected as solar activity rises towards the next maximum. However, this task will be challenging not only by the continuing variations aboard the satellite, which in turn affect the polarimetric measurements, but also by the comparatively low frequency with which the quiet Sun 
magnetic fields on disk centre are recorded by SOT/SP. This problem is compounded by the fact that the solar activity is concentrated ever closer to the solar equator as cycle 24 progresses, making it difficult to ascertain the nature of the quiet Sun at the disk centre during the activity maximum. Hence, it will be interesting to investigate to what extend the Helioseismic and Magnetic Imager (HMI, Schou et al.2012b a) aboard the Solar Dynamic Observatory (SDO) can follow internetwork magnetic flux. Its larger field-of-view is a major advantage, which might overcome some of the disadvantages caused by its lower spatial resolution. Since SDO was launched in 2010, it unfortunately missed the last activity minimum, but will be an interesting instrument to follow solar flux in the future. Nonetheless, the comparatively high spatial and spectral resolution of Hinode SOT/SP and its ability to measure all four Stokes parameters, make it a very promising instrument with which to continue investigating the internetwork magnetic field in such detail whilst covering a period of time comparable to a solar cycle.

Table 2.1: Hinode SOT/SP images

\begin{tabular}{ccccc}
\hline \hline Image & Date & UT & X, Y & Area $^{2}$ \\
\hline 1 & 2006 Nov 26 & $13: 14$ & $-178,-56$ & $325 \times 162$ \\
2 & 2006 Dec 19 & $11: 35$ & $-274,-1$ & $220 \times 162$ \\
3 & 2007 Feb 18 & $03: 20$ & $-89,-1$ & $160 \times 162$ \\
4 & 2007 Apr 17 & $15: 04$ & $-57,1$ & $110 \times 162$ \\
5 & 2007 May 20 & $16: 43$ & $-56,1$ & $82 \times 81$ \\
6 & 2007 Sep 10 & $07: 20$ & $-41,-1$ & $48 \times 162$ \\
7 & 2007 Oct 15 & $17: 21$ & $-164,-1$ & $325 \times 162$ \\
8 & 2007 Dec 21 & $00: 20$ & $-81,-1$ & $164 \times 162$ \\
9 & 2008 Jan 30 & $23: 34$ & $-34,35$ & $60 \times 130$ \\
10 & 2008 Oct 22 & $07: 14$ & $-14,24$ & $30 \times 130$ \\
11 & 2008 Oct 22 & $08: 14$ & $-14,-124$ & $30 \times 130$ \\
12 & 2008 Nov 19 & $03: 40$ & $-14,130$ & $30 \times 130$ \\
13 & 2008 Nov 19 & $04: 10$ & $-14,0$ & $30 \times 130$ \\
14 & 2008 Dec 17 & $04: 34$ & $-14,134$ & $30 \times 130$ \\
15 & 2008 Dec 17 & $05: 43$ & $-14,-35$ & $30 \times 130$ \\
16 & 2009 Feb 17 & $22: 34$ & $-14,-31$ & $30 \times 130$ \\
17 & 2009 Feb 17 & $23: 34$ & $-14,131$ & $30 \times 130$ \\
18 & 2009 Apr 11 & $18: 05$ & $-14,0$ & $30 \times 130$ \\
19 & 2009 Apr 11 & $16: 20$ & $-14,120$ & $30 \times 130$ \\
20 & 2009 May 20 & $18: 42$ & $-14,88$ & $30 \times 130$ \\
21 & 2009 May 20 & $19: 07$ & $-14,-88$ & $30 \times 130$ \\
22 & 2009 Jul 28 & $15: 29$ & $-14,-5$ & $30 \times 130$ \\
23 & 2009 Jul 28 & $14: 07$ & $-14,-204$ & $30 \times 130$ \\
24 & 2009 Sep 01 & $20: 58$ & $-14,-11$ & $30 \times 130$ \\
25 & 2009 Sep 01 & $21: 48$ & $-14,111$ & $30 \times 130$ \\
26 & 2009 Oct 05 & $16: 04$ & $-24,-29$ & $30 \times 130$ \\
27 & 2009 Oct 05 & $16: 54$ & $-24,108$ & $30 \times 130$ \\
\hline & & & &
\end{tabular}


Table 2.1: Hinode SOT/SP images

\begin{tabular}{ccccc}
\hline \hline Image & Date & UT & X, Y & Area \\
\hline 28 & 2009 Dec 21 & $21: 34$ & $-24,125$ & $30 \times 130$ \\
29 & 2009 Dec 21 & $19: 59$ & $-24,-45$ & $30 \times 130$ \\
30 & 2010 Jan 28 & $23: 21$ & $-30,27$ & $30 \times 130$ \\
31 & 2010 Jan 29 & $00: 34$ & $-30,227$ & $30 \times 130$ \\
32 & 2010 Feb 23 & $19: 58$ & $-30,-37$ & $30 \times 130$ \\
33 & 2010 Feb 23 & $21: 36$ & $-30,123$ & $30 \times 130$ \\
34 & 2010 Apr 29 & $02: 55$ & $-30,138$ & $30 \times 130$ \\
35 & 2010 Apr 29 & $01: 39$ & $-30,-52$ & $30 \times 130$ \\
36 & 2010 May 31 & $01: 40$ & $-22,-72$ & $30 \times 130$ \\
37 & 2010 May 31 & $02: 58$ & $-22,118$ & $30 \times 130$ \\
38 & 2010 Jul 03 & $20: 24$ & $-19,112$ & $30 \times 130$ \\
39 & 2010 Jul 03 & $18: 47$ & $-19,-78$ & $30 \times 130$ \\
40 & 2010 Aug 31 & $14: 19$ & $-14,-145$ & $30 \times 130$ \\
41 & 2010 Aug 31 & $15: 09$ & $-14,45$ & $30 \times 130$ \\
42 & 2010 Oct 07 & $14: 43$ & $-25,-160$ & $30 \times 130$ \\
43 & 2010 Oct 07 & $16: 07$ & $-25,30$ & $30 \times 130$ \\
44 & 2010 Nov 11 & $16: 31$ & $-25,30$ & $30 \times 130$ \\
45 & 2010 Nov 11 & $15: 07$ & $-25,-160$ & $30 \times 130$ \\
46 & 2010 Dec 12 & $11: 04$ & $-85,-1$ & $160 \times 162$ \\
47 & 2011 Jan 05 & $11: 53$ & $-26,-55$ & $30 \times 130$ \\
48 & 2011 Jan 05 & $12: 43$ & $-26,135$ & $30 \times 130$ \\
49 & 2011 Feb 02 & $13: 47$ & $-26,-55$ & $30 \times 130$ \\
50 & 2011 Feb 02 & $14: 37$ & $-26,135$ & $30 \times 130$ \\
51 & 2011 Mar 01 & $14: 06$ & $-14,-145$ & $30 \times 130$ \\
52 & 2011 Mar 01 & $14: 56$ & $-14,45$ & $30 \times 130$ \\
53 & 2011 May 03 & $18: 48$ & $-30,-58$ & $30 \times 130$ \\
54 & 2011 May 03 & $20: 25$ & $-30,132$ & $30 \times 130$ \\
55 & 2011 Jun 02 & $20: 55$ & $-20,-82$ & $30 \times 130$ \\
56 & 2011 Jun 02 & $22: 31$ & $-20,108$ & $30 \times 130$ \\
57 & 2011 Jun 30 & $20: 38$ & $-18,4$ & $30 \times 130$ \\
58 & 2011 Jun 30 & $19: 01$ & $-18,-186$ & $30 \times 130$ \\
59 & 2011 Aug 02 & $22: 32$ & $-21,117$ & $30 \times 130$ \\
60 & 2011 Aug 02 & $20: 56$ & $-21,-73$ & $30 \times 130$ \\
61 & 2011 Nov 30 & $20: 01$ & $-24,-159$ & $30 \times 130$ \\
62 & 2011 Nov 30 & $21: 38$ & $-24,29$ & $30 \times 130$ \\
63 & 2011 Dec 27 & $23: 00$ & $-24,159$ & $30 \times 130$ \\
64 & 2011 Dec 27 & $23: 46$ & $-24,-29$ & $30 \times 130$ \\
65 & 2012 Feb 02 & $16: 46$ & $-28,160$ & $30 \times 130$ \\
66 & 2012 Feb 02 & $15: 22$ & $-28,-59$ & $30 \times 130$ \\
67 & 2012 Feb 28 & $14: 57$ & $-27,-60$ & $30 \times 130$ \\
68 & 2012 Feb 28 & $16: 21$ & $-27,130$ & $30 \times 130$ \\
69 & 2012 Mar 31 & $00: 58$ & $-27,-60$ & $30 \times 130$ \\
\hline & & & & \\
\hline 5
\end{tabular}


Table 2.1: Hinode SOT/SP images

\begin{tabular}{ccccc}
\hline \hline Image & Date & UT & $\mathrm{X}, \mathrm{Y}^{1}$ & Area $^{2}$ \\
\hline 70 & 2012 Mar 31 & $02: 34$ & $-27,130$ & $30 \times 130$ \\
71 & 2012 May 03 & $17: 28$ & $-26,-62$ & $30 \times 130$ \\
72 & 2012 May 03 & $19: 05$ & $-26,127$ & $30 \times 130$ \\
& & & & \\
\hline
\end{tabular}

1 Commanded position, E,N arcseconds of disc centre. May vary from actual position.

${ }^{2} \mathrm{X}, \mathrm{Y}$ arcseconds 



\section{Properties of solar plage from a spatially coupled inversion of Hinode SP data}

\subsection{Abstract}

The properties of magnetic features composing a plage region in the vicinity of a sunspot were investigated at high spatial resolution. Stokes spectra of the $630 \mathrm{~nm}$ line pair recorded by the spectropolarimeter aboard Hinode were inverted using an extended version of the SPINOR code. The code preformed a spatially coupled inversion of the Stokes spectra using three $\log (\tau)$ nodes in optical depth, allowing the retrieval of the height dependence of physical parameters in each pixel of the data set, with the effect of the instrument's point spread function removed. No magnetic filling factors was employed. The analysis of the inversion results reveals that the plage is composed of magnetic flux concentrations (MFCs) with typical field strengths of $1490 G$ at $\log (\tau)=-0.9$ and inclinations between $10^{\circ}-15^{\circ}$ in all three $\log (\tau)$ nodes. We stress that these results were obtained without introducing a magnetic filling factor. The field strength in MFCs drops less rapidly with height than expected from the zeroth order thin-tube approximation, but the expansion of the magnetic field is in good agreement with the thin tube model. Whilst the gas inside magnetic flux concentrations is typically at rest, the majority of MFCs were surrounded by a ring of downflows with typical values of $1-3 \mathrm{~km} / \mathrm{s}$. The MFCs expand by forming magnetic canopies composed of weaker and more inclined magnetic fields. While the temperature stratification of MFCs is consistent with an emperical model of plage flux tubes. Within the downflow rings of MFCs small magnetic patches of weak opposite polarity to that of the main MFC were identified, which are predominantly situated beneath the canopy of the main MFC. Magnetic fields found close to the sunspot showed more inclined fields and an azimuthal orientation either away or towards the spot depending on their polarity. We found evidence for a strong broadening of the Stokes profiles in MFCs (expressed by a microturbulence in the inversion). This indicates the presence of strong unresolved velocities.

\subsection{Introduction}

In a typical active region on the solar disc three features can be identified most easily at visible wavelengths: sunspots, pores and plages. Whilst sunspots and pores are defined 
by their characteristic darkening of the continuum intensity, plages appear brighter than the surrounding quiet Sun mainly in spectral lines, or as faculae near the solar limb in the continuum. It has been known since the work of Hale (1908) that sunspots and pores harbour magnetic fields on the order of $k G$. Babcock and Babcock (1955) showed that plage, too, is associated with magnetic fields, but it was only realized much later that it is also predominantly composed of $k G$ magnetic fields (Howard and Stenflo 1972; Frazier and Stenflo 1972; Stenflo 1973).

The $k G$ magnetic fields, or magnetic flux concentrations (MFCs), in plage are often considered to take the form of small flux tubes or sheets and considerable effort has gone into determining their structure and dynamics in detail, see the review by Solanki (1993). The convective collapse mechanism (Parker 1978, Spruit 1979) is thought to concentrate the field to $\mathrm{kG}$ values (Nagata et al. 2008; Danilovic et al. 2010c), whereby the plasma inside the tube is evacuated and the magnetic field is concentrated (Grossmann-Doerth et al. 1998, Venkatakrishnan 1986). The diameter of an individual $k G$ flux tube is expected to be a few $100 \mathrm{~km}$ or less. In the quiet Sun, diameters typically do not exceed $100 \mathrm{~km}$ necessitating an instrument with an angular resolution of $0 . " 15$ or less to fully resolve an individual flux tube (Lagg et al.2010). Due to the comparatively small lateral size of these flux tubes, they are commonly treated using a thin flux tube model (Spruit 1976, Defouw 1976), where the lateral variation of the atmospheric parameters inside the tube is smaller than the pressure scale height. 3-D radiative MHD simulations (Vögler et al. 2005) give rise to magnetic concentrations with properties that are close to those of the thin-tube approximation (Yelles Chaouche et al.2009). More complex flux-tube models have also been investigated (see Zayer et al. (1989) and refs. therein).

Despite their small size many of the general properties of flux tubes residing in plages have nonetheless been determined by observations. This has been achieved by analyzing the polarization of the light that is produced by the Zeeman effect in areas containing magnetic field (Solanki 1993). Thus, e.g. Rabin (1992); Zayer et al. (1990) and Rüedi et al. (1992) found, using the deep photospheric infrared Fe I $1.56 \mu \mathrm{m}$ line, magnetic field strengths of $1400-1700 \mathrm{G}$, when taking into account that the magnetic field is not resolved. Field strengths of around 1400G were obtained by Wiehr (1978) and Martínez Pillet et al. (1997) by using lines in the visible such as the $630 \mathrm{~nm}$ line pair, whilst values of only $1000-1100 G$ were found by Stenflo and Harvey (1985) with the 525nm lines, which are formed higher in the photosphere. Finally, the Mg I $12.3 \mu \mathrm{m}$ lines, used by Zirin and Popp (1989) returned values as low as $200-500 G$ in plage, which are fully consistent with the $k G$ fields observed in other lines (Bruls and Solanki 1995) due to the even higher formation height of these lines.

The inclination of MFCs in plage was found to be predominantly vertical, with typical inclinations of $10^{\circ}$, due to the magnetic buoyancy of the flux tubes (Schüssler 1986), although MFCs with highly inclined magnetic fields were also found (Topka et al. 1992; Bernasconi et al. 1995; Martínez Pillet et al.|1997). The azimuthal orientation of MFCs was shown to have no preferred direction (Martínez Pillet et al. 1997) and form so called 'azimuth centres', however Bernasconi et al. (1995) did find a preferred $E-W$ orientation. The potential existence of mass motions inside magnetic elements has been fueled by the observation of significant asymmetries in the Stokes $Q, U$ and particularly $V$ profiles, in both amplitude and area (Solanki and Stenflo 1984). However, Solanki (1986) showed that within a magnetic element such as MFCs no stationary mass motions are present and only 
weak downflows of on average $200 \mathrm{~m} / \mathrm{s}$ can be observed. This result was confirmed by Martínez Pillet et al. (1997) using a Milne-Eddington (ME) inversion. In particular, the area asymmetries in the Stokes profiles are thought to result from the interplay between the magnetic element and the convecting plasma in which it is immersed (Grossmann-Doerth et al. 1988; Solanki 1989). Briand and Solanki (1998) showed that profiles of the $\mathrm{Mg} \mathrm{I} b_{2}$ line can be fitted with atmospheres representing a flux tube expanding with height, containing no significant flows, whilst being surrounded by strong downflows of up to $5 \mathrm{~km} / \mathrm{s}$ from the convecting plasma around it. This scenario is supported by magneto-hydrodynamic simulations performed by e.g. Deinzer et al. (1984); Grossmann-Doerth et al. (1988); Steiner et al. (1996); Vögler et al. (2005).

More recently observations at higher spatial resolutions have further confirmed this picture (Rouppe van der Voort et al. 2005). Langangen et al. (2007) found, by placing a slit across a plage-like feature, downflows in the range of $1-3 \mathrm{~km} / \mathrm{s}$ at the edges of the feature. Cho et al. (2010) observed, using SOT/SP, that pores, too, are surrounded by strong downflows in the photosphere.

The relationship between the magnetic field strength and continuum intensity of plage was studied extensively by Kobel et al. (2011) and a clear dependence of the continuum intensity on the magnetic field strength was found. Furthermore, the granular convection in plage has an abnormal appearance in areas where plage is present (Title et al. 1989). Morinaga et al. (2008) and Kobel et al. (2012) concluded that the high spatial density of the $k G$ magnetic fields causes a suppression of the convection process. Narayan and Scharmer (2010) also found a convection pattern four times smaller than the normal granulation in areas containing plage.

The expansion of MFCs has also been investigated. Pietarila et al. (2010) used SOT/SP images at various $\mu$-values and examined the change in sign of the Stokes $V$ signal of magnetic fields in the quiet network observed near the limb. The variations of the Stokes $V$ signal across the MFC was, with help of MHD simulations, found to be compatible with the thin flux tube approximation. Martínez González et al.(2012) analyzed the Stokes $V$ area asymmetry across a network patch recorded with IMaX (Martínez Pillet et al.2011) aboard SunRISE (Solanki et al. 2010; Barthol et al. 2011) on the disk centre and found that the internal structure of the large network patch was likely to be more complex than that of a simple thin flux tube approximation. A similar conclusion concerning the internal structure of plages was reached by Berger et al. (2004). Rezaei et al. (2007) also examined the change of the Stokes $V$ area asymmetry of a network patch situated at the disk centre using SOT/SP and showed that it was surrounded by a magnetic canopy. Yelles Chaouche et al. (2009) analyzed thin flux tubes and sheets produced by MHD simulations and concluded that a $2^{\text {nd }}$ order flux tube approximation is necessary to accurately describe the structure of the magnetic features. Solanki et al. (1999) showed that the expansion of sunspot canopies is close to that of a thin flux tube and further went on to show that the relative expansion of all flux tubes is similar. It is the relative expansion of magnetic features that the remainder of this section will focus on.

As illustrated by the above papers, which are only a small sample of the rich literature on this topic, there has been significant progress in our knowledge of plage in the last decade. Nonetheless, no comprehensive study of plage properties using inversions has been published since the work of Martínez Pillet et al. (1997), which was based on 1" resolution data from the Advanced Stokes Polarimeter (ASP). In the following sections we 
aim to both test and expand upon our knowledge of the typical characteristics associated with plage using the results provided by the recently developed and powerful spatially coupled inversion method (van Noort 2012) applied to Hinode SOT/SP observations. We concentrate here on the strong-field magnetic elements and do not discuss the horizontal weak-field features also found in active region plage areas (Ishikawa et al. 2008; Ishikawa and Tsuneta 2009).

\subsection{Data}

The data set used in this investigation was recorded by the solar optical telescope (SOT/SP) (Tsuneta et al. 2008b; Suematsu et al. 2008; Ichimoto et al. 2008; Shimizu et al. 2008) aboard Hinode (Kosugi et al. 2007) on the $30^{\text {th }}$ of April 2007, UT 18:35:18 - 19:39:53. The region was scanned in normal mode with a total exposure time of $4.8 s$ per slit position and an angular resolution of 0." 3 . All four Stokes parameters, $I, Q, U$ and $V$, were recorded at each slit position. The field of view contains a fully developed sunspot of the active region (AR) 10953 with large areas of plage trailing it. During the observation the spot was located in the southern hemisphere towards the east limb, $-190 \mathrm{X},-200 Y$, at $\mu=0.97$ ( $\mu=\cos (|\theta|)$, where $\theta$ is the heliocentric angle). A normalized continuum image of the investigated region used in the inversion is shown in Fig. 3.1. The data were reduced using the standard sp_prep routine (Lites et al.2008) from the solar software package, followed by a bitshift correction of the four Stokes parameters.

\subsection{Inversions}

The region of the SOT/SP scan containing most of the plage was inverted using the SPINOR code (Frutiger et al. 2000), which uses response functions in order to perform a least-squares fitting of the Stokes spectra. It is based upon the STOPRO routines described by Solanki et al. (1987). The SPINOR code was extended by van Noort (2012) to perform spatially coupled inversions using the point-spread-function (PSF) of the SOT/SP. Such spatially coupled inversions have already been successfully applied to Hinode SOT/SP data of sunspots by Riethmüller et al. (2013); van Noort et al. (2013); Tiwari et al. (2013). We employ the same PSF used by them, based on the work of Danilovic et al. (2008). The size of the inverted area, corresponding to that shown in Fig. 3.1, is the largest that can be inverted by the employed code in one go due to computing limitations. The inversion code allows the recovery of thermal, magnetic and velocity stratifications, which are indicated by the strengths, shapes and asymmetries present in the SOT/SP Stokes profiles (Solanki 1993, Viticchié and Sánchez Almeida 2011; Stenflo 2010). These stratifications are recovered by setting multiple nodes in $\log (\tau)$ at which the various atmospheric parameters can be varied. The functions of each atmospheric parameter with optical depth $\log (\tau)$ are calculated using a spline interpolation through the $\log (\tau)$ nodes. These functions are then used to solve the radiative transfer equation and the emergent spectra are fitted iteratively by a Levenberg-Marquardt algorithm that minimizes the $\chi^{2}$ merit function.

The setting of the nodes in $\log (\tau)$ is important in order to achieve a realistic atmospheric stratification. With the help of response functions it is possible to determine the optical 
depths where the spectral line profile changes in response to a change in the atmosphere. Three nodes were set at optical depths $\log (\tau)=0,-0.9$ and -2.3 based on the response functions of the $630 \mathrm{~nm}$ line pair. The response functions were calculated for an atmosphere simulating a plage pixel i.e. containing magnetic field of $2000 G$ at $\log (\tau)=0$ and satisfying the thin-tube approximation at all heights. At each of the three chosen nodes the temperature, T, magnetic field strength, B, inclination relative to the line-of-sight (los), $\gamma$, azimuth, $\psi$, line-of-sight velocity, v, and micro turbulence, $\xi_{\text {mic }}$, were fitted, leading to 18 free parameters in total. We stress that no macro turbulence broadening was allowed and a general $\mu$-value of $\mu=0.97$ of the observation was taken into account when inverting. Also, no magnetic filling factor was introduced in the inversion. The influence of straylight from neighbouring pixels is taken into account properly by the code, while we assume that each pixel is filled with the properties returned by the inversion code.

A problem affecting any inversion process is the possibility that the fitting algorithm finds a solution that corresponds to a local minimum. This is particularly so if the initial guess atmosphere for a pixel provided to the algorithm is far from the global minimum. In an effort to ensure that the solution for each pixel of the inversion corresponds to the global minimum, the inversion process was performed a total of four times with each inversion performing 12 iterations. Save for the initial inversion each successive inversion used the smoothed results of the previous inversion as an initial input, thereby ensuring that the initial guess for each pixel is closer to the global minimum. After the fourth inversion process the mean $\chi^{2}$ value of all the pixels could not be decreased any further as was observed when the scan was inverted a successive time.

\subsection{Results}

In this section we describe the various results obtained with the inversion. First we give a general overview of the output of the inversion followed by subsections dealing with more specific points. Figures $3.1 \& 3.2$ provide an overview of the continuum intensity, the magnetic field strength returned by the inversions (Figs. 3.2a-c) and the LOS inclination of the magnetic field vector (Figs. 3.2d-f), i.e. the inclination relative to the line-of-sight. Fig. 3.1 shows part of the sunspot's penumbra and pores within the field of view to which the inversion code was applied. Many of the pores in the figure are only a few pixels in size as illustrated by the red contour line. However, Figs. 3.2a-c show that in terms of magnetic field strength many pores, indicated by the black contour lines encompassing pixels where $I_{c}<0.8\left\langle I_{c}\right\rangle$, are indistinguishable from plages in the image and are often entirely embedded in a larger magnetic feature. Figures $3.2 \mathrm{~d}-\mathrm{f}$ are also consistent with the view that the transition from plage to pore and vice versa is a continuous and common occurrence (Grossmann-Doerth et al. 1994, Berger et al. 2004). However, the aim of this paper is to investigate the (time-independent) properties of plage, thus the pores in the image are not discussed further.

Figures 3.2 d-f show that most of the plage has the same, positive, polarity (shown in blue). Only in the lower right hand corner of the field-of-view (FOV) can plage of the opposite polarity be found (shown in red in Figs. 3.2 d-f). The dominant polarity of the plage is opposite to that of the sunspot. Between the plage of opposite polarities a polarity inversion line (PIL) can be seen, stretching from approximately $-212 X,-245 Y$ to $-227 X$, 


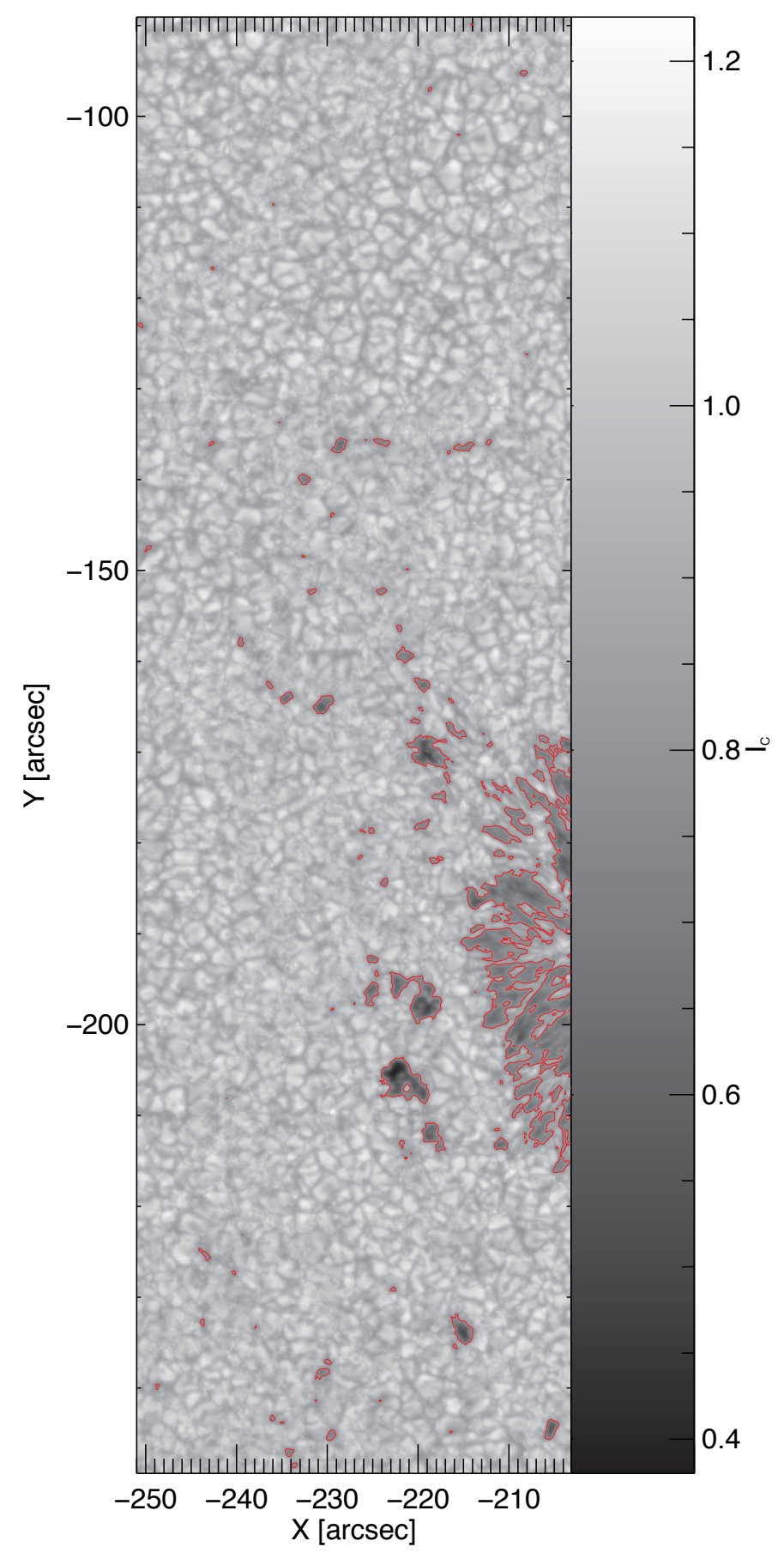

Figure 3.1: Normalized continuum intensity image of the region in which the Stokes profiles were inverted. Pixels where $I_{c}<0.8\left\langle I_{c}\right\rangle$ are enclosed by the red contour. The $x$ and $y$ axes indicate the distance to the solar equator and central meridian, respectively. 

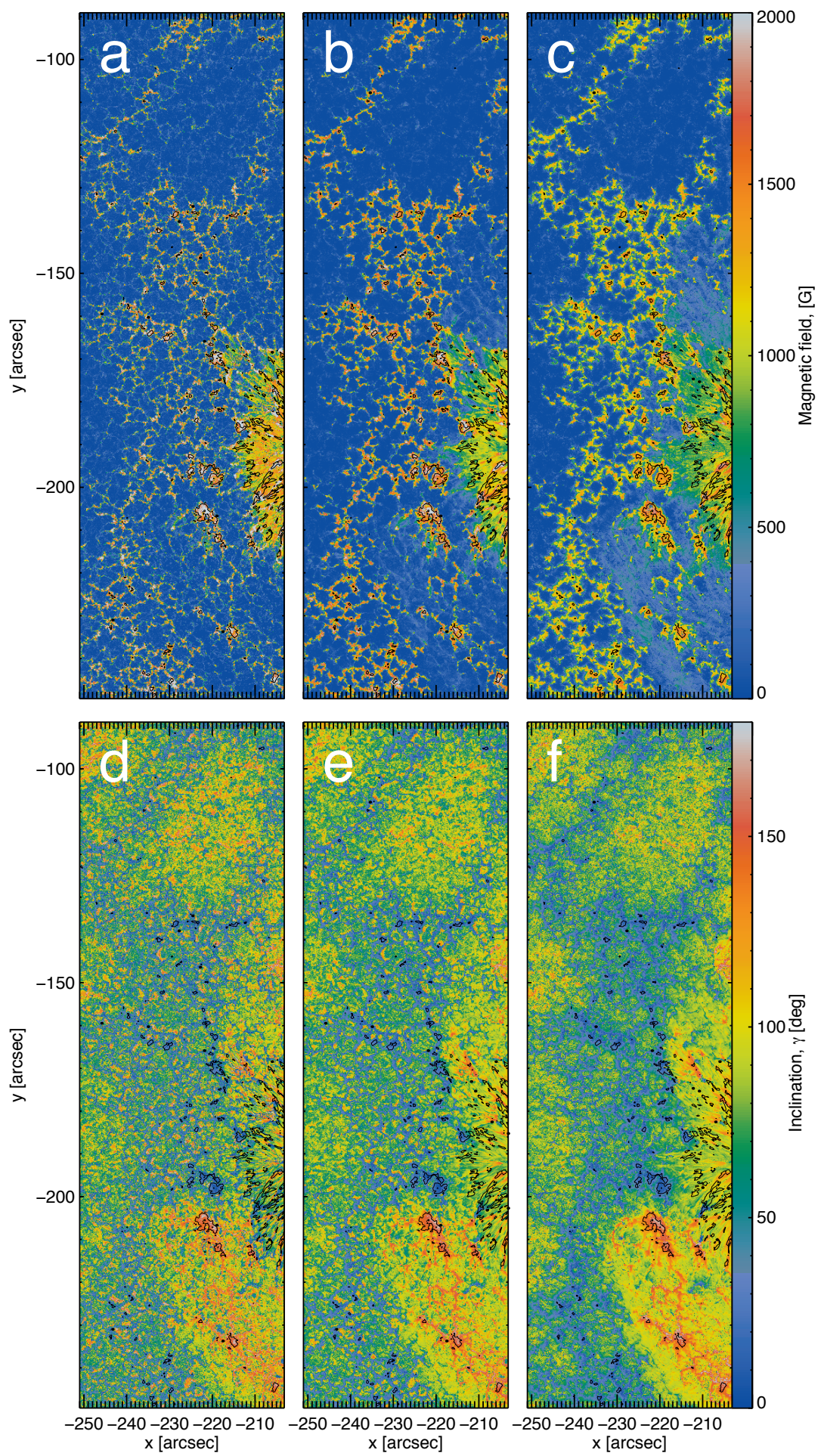

$-250-240-230-220-210-250-240-230-220-210$
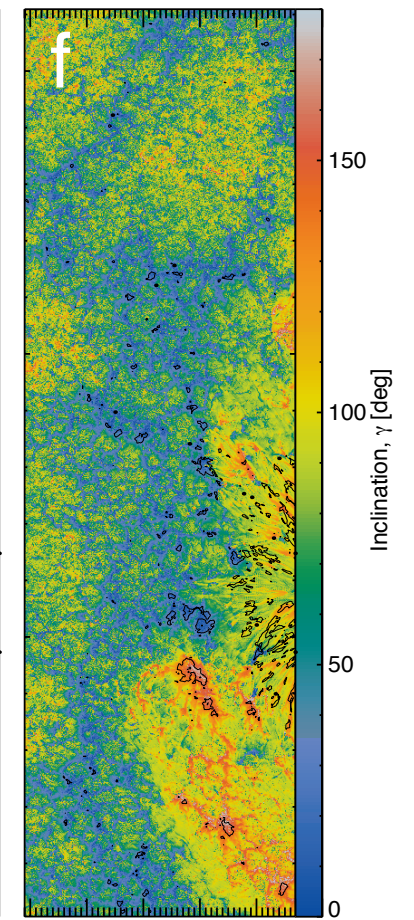

$\mathrm{x}[\operatorname{arcsec}]$

$\mathrm{x}[\mathrm{arcsec}]$

Figure 3.2: $a-c$ : The magnetic field strength retrieved by the inversion at $\log (\tau)=0,-0.9$ and -2.3 from left to right. The colour scale given on the right is identical in all the three images. $d$-f: The line-of-sight inclination of the magnetic field obtained by the inversion at $\log (\tau)=0,-0.9$ and -2.3 from left to right. All the three images share the same colour scale. The black contour in all images encompasses pixels where $I_{c}<0.8\left\langle I_{c}\right\rangle$. 
$-210 Y$ in Figs. 3.1 \& 3.2. H $\alpha$ images not shown here indicate the presence of a filament along this PIL. The lower part of this filament is visible in Fig. 3.2 at $\log (\tau)=-2.3$ as predominantly horizontal magnetic fields (Fig. 3.2f) of around 350G. The atmosphere below the PIL is almost free of magnetic field (Fig. 3.2 ). For a more detailed analysis of this filament the reader is referred to Okamoto et al. (2008, 2009). Here we can add to their findings that although the filament magnetic field reaches down into the photosphere, it is largely restricted to layers more than roughly $200 \mathrm{~km}$ above the solar surface. We converted from optical depth to a height scale by assuming hydrostatic equilibrium and the temperature stratification returned by SPINOR. The B value of $350 G$ in the filament is comparable (within a factor of 2) to the field strengths found in AR filaments by Xu et al. (2010); Kuckein et al. (2012); Sasso et al. (2011) in the chromosphere sampled by the He I $10830 \AA$ triplet. The azimuthal orientation of the magnetic field within the filament is almost invariant across the whole filament, as expected, and makes an angle of $90^{\circ}$ with the radial orientation to the sunspot. Also, the orientation is almost parallel to the axis of the PIL. This excludes the possibility that the filament could be a low lying part of the sunspot's canopy.

Figures $3.2 \mathrm{a}-\mathrm{c}$ indicate that almost all magnetic features appear to expand quite significantly with height. This suggests that many pixels contain magnetic fields only in the higher layers of the atmosphere and form magnetic canopies. Therefore, all plage pixels were divided into two populations: core pixels and canopy pixels. The core pixels were defined by a positive magnetic field gradient with optical depth and an absolute magnetic field strength, $B,>900 G$ at $\log (\tau)=0$. Pixels with $B<900 G$ at $\log (\tau)=0$ often had magnetic fields $<100 G$ at $\log (\tau)=-2.3$ and were therefore considered too weak to be counted as plage. Results from a higher threshold at $\log (\tau)=0$ merely reduced the number of selected pixels but qualitatively did not differ from the results presented here. The canopy pixels were defined by a negative magnetic field gradient with optical depth, i.e. an increasing field strength with height, and an absolute magnetic field strength, $B,>300 G$ at $\log (\tau)=-2.3$. A threshold $<300 G$ at $\log (\tau)=-2.3$ caused the selection of a large number of pixels that were not directly connected to plage. These 'extra' pixels were predominantly weak horizontal magnetic fields found atop of granules in the few quiet Sun areas in Fig. 3.1. These are likely related to the weak horizontal fields found in plage by Ishikawa and Tsuneta (2009). All the pixels selected by the two thresholds had a Stokes $Q, U$ or $V$ amplitude of at least $5 \sigma$, where $\sigma=1 \times 10^{-3} I_{c}$. Small pores embedded within plage were discarded by setting an intensity threshold, $I_{c}>0.8\left\langle I_{c}\right\rangle$. The location of core and canopy pixels using these thresholds is illustrated in Fig. 3.3. The figure shows that the canopy pixels surround the core pixels. The location of the filament is clearly seen as the elongated canopy-like structure following the PIL. The sunspot's canopy produces a grey ring bordering the sunspot and forms loops between the sunspot and adjacent, opposite polarity pores. Such a loop structure is located at approximately $-215 X,-200 Y$. The sunspot's canopy extends particularly far, as elongated finger-like structures at $-210 X$, $-160 Y$. These fingers are presumably very low lying loops connecting the spot to the plage elements. The clear division between these various magnetic structures is only possible by applying the inversion code in 2D coupled mode.

Fig. 3.3 demonstrates the change in size of the MFCs when looking at different layers in $\log (\tau)$. However, not only the magnetic field changes with height but rather all the parameter returned by the inversion show a height dependence. This can be illustrated by 


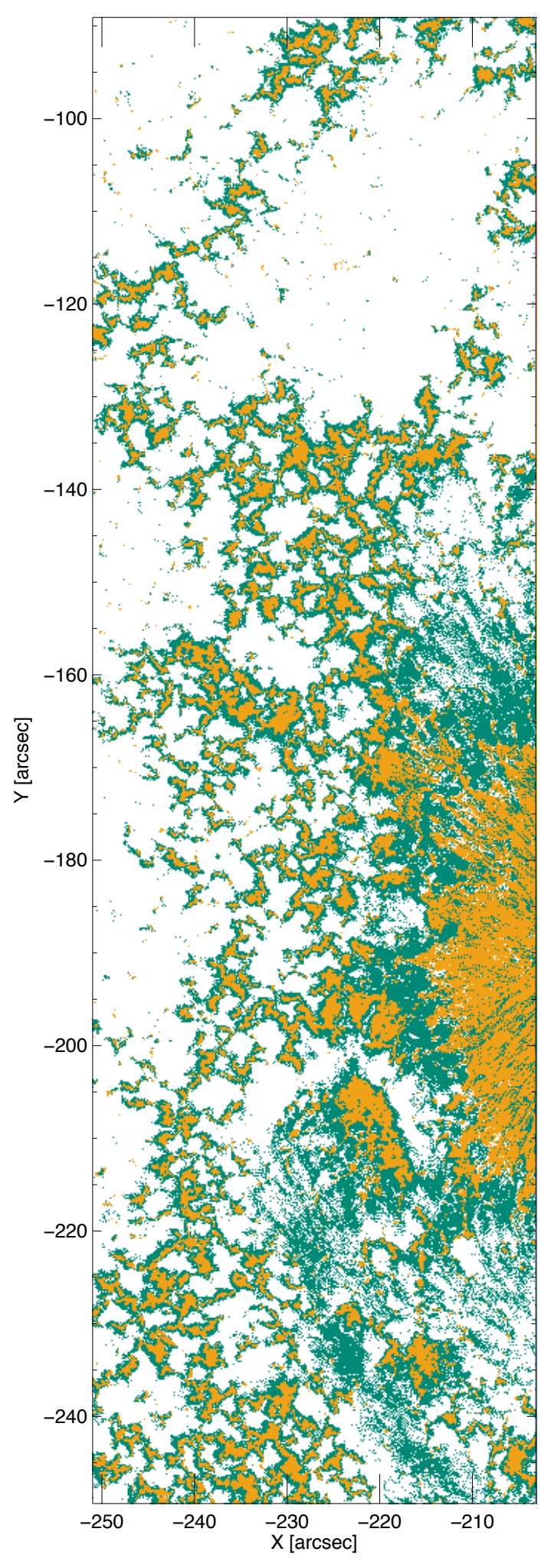

Figure 3.3: Image showing the location of core and canopy pixels using the definition given in Sect. 3. Core pixels are shown in white and canopy pixels are coloured in grey. The black areas contain weak magnetic fields that were not considered as belonging directly to the plage. 

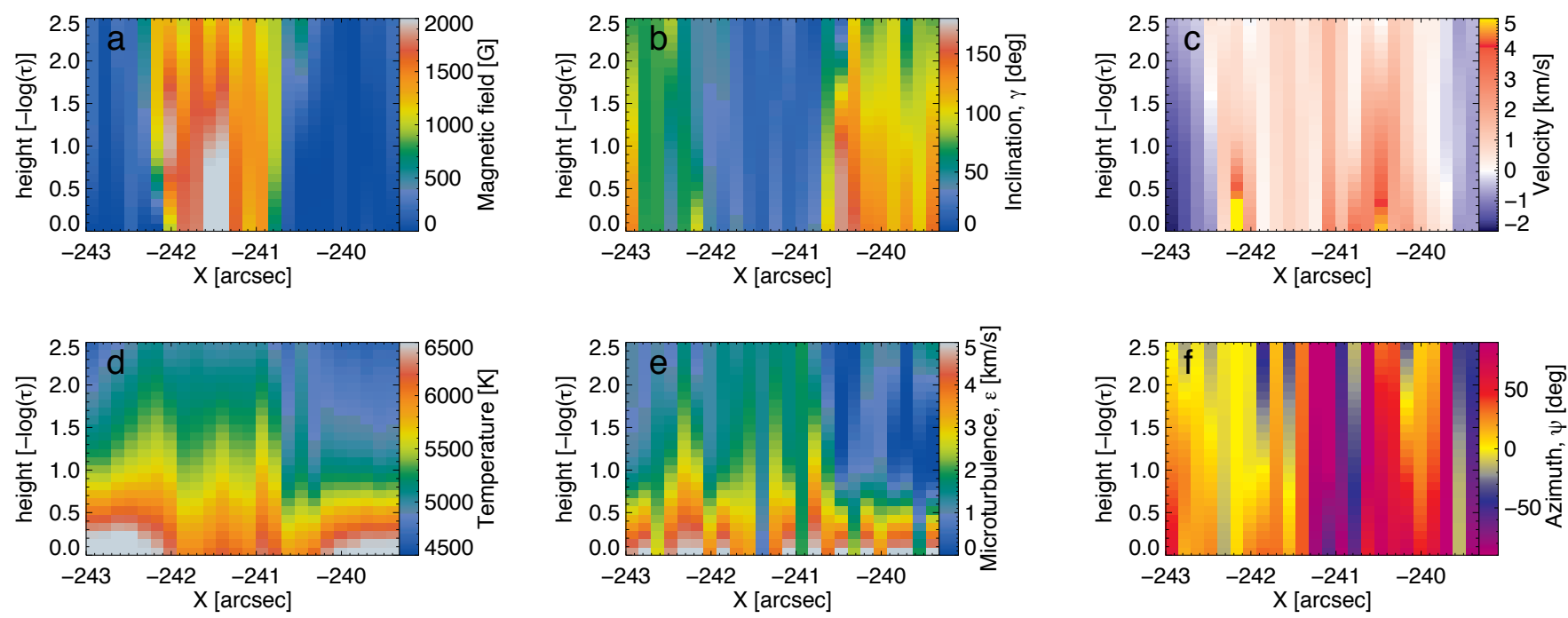

Figure 3.4: A vertical slice through a typical MFC. The Y coordinate of this MFC is -154". $a-c$ : Magnetic field, LOS inclination and LOS velocity from left to right. $d$-f: Temperature, microturbulence and azimuth from left to right.

examining a vertical cut through a MFC, as is depicted in Fig. 3.4. The $\log (\tau)$ values for all parameters at heights other than the three preset nodes were obtained by a cubic spline interpolation between the nodes, which was also performed during the inversion process. The figure already displays many of the features that will be investigated in more detail in the following sections. The MFC in Fig. 3.4 is composed of nearly vertical $k G$ magnetic fields that are decreasing with height between $-241 X$ to $-242 X$, whilst the feature is expanding and is surrounded by strong downflows on either side near $\log (\tau)=0$. Both the temperature and the microturbulence are enhanced at mid-photospheric layers within the MFC with respect to the surrounding quiet Sun. The feature lies between two granules, which can be identified easily in the temperature image at $\log (\tau)=0$. The pixel-to-pixel variations seen in Fig. 3.4 sizeable, but statistically the results are quite robust, as Figs. 3.2 $\& 3.3$ display.

\subsubsection{Magnetic field strength}

Figures $3.2 \mathrm{a}$-c indicate that plage is composed of magnetic fields on the order of $k G$. This is shown quantitatively by histograms of magnetic field strength in Fig. 3.5 using the magnetic field thresholds defined in Sect. 3. Besides histograms of B of core plage fields at each optical depth, the histogram of B of the canopy pixels at $\log (\tau)=-2.3$ is shown as well. Histograms of the magnetic field strength for the canopy at $\log (\tau)=0$ and -0.9 have been omitted as at these heights the atmosphere is similar to the quiet Sun. Fig. 3.5 shows that at $\log (\tau)=-0.9$ the magnetic field strength peaks between $1400 G$ and $1500 G$ with an average value of $1490 \mathrm{G}$. At this height the two Fe I absorption lines show the greatest response to all the fitted parameters, making the results from this node the most comparable to results obtained from Milne-Eddington (ME) inversions. Similar averages of intrinsic magnetic field strengths for plages from inversions have been reported by Martínez Pillet et al. (1997); Keller et al. (1990); Zayer et al. (1990) using absorption 


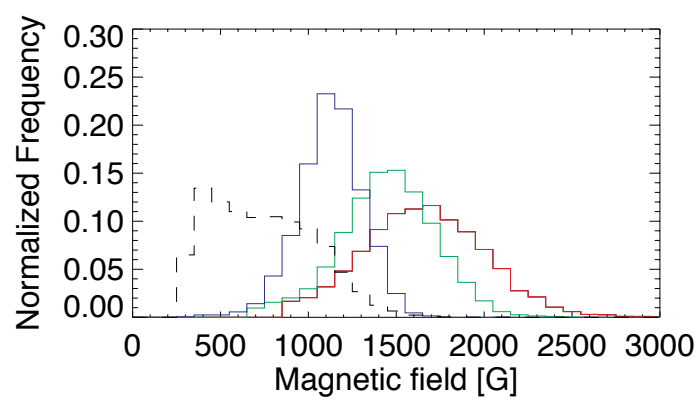

Figure 3.5: Histograms of B values found in plages. The three coloured histograms were obtained using core pixels, where red refers to $\log (\tau)=0$, green indicates $\log (\tau)=-0.9$ and blue refers to $\log (\tau)=-2.3$. The dashed histogram shows the field strengths of canopy pixels at $\log (\tau)=-2.3$.

lines in the visible. Whereas these authors needed to introduce a magnetic filling factor to obtain such high intrinsic field strengths, our B values are obtained without a filling factor. This, and the good agreement with the results of Rabin (1992); Rüedi et al. (1992) obtained using the extremely Zeeman sensitive Fe I 1.56 micron lines, demonstrates that the combination of Hinode SOT/SP data and the spatially coupled inversion technique is capable of resolving most of the strong-field features in AR plage. As expected, the average magnetic field strength in core pixels decreases with decreasing optical depth, so whilst at $\log (\tau)=0$ the average field strength is $1699 G$ at $\log (\tau)=-2.3$ the average field strength drops to $1169 \mathrm{G}$, which is consistent with field strengths in plage obtained

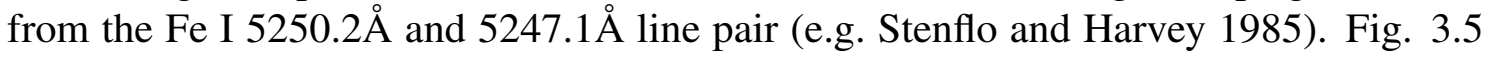
also shows that the widths of the histograms using core pixels decreases with height. At $\log (\tau)=0$ the FWHM is $810 G$ and then subsequently decreases to $640 G$ at $\log (\tau)=-0.9$. The FWHM at $\log (\tau)=-2.3$ is $420 G$, i.e. roughly half the value measured at $\log (\tau)=0$. The comparatively broad distribution at $\log (\tau)=0$ may be intrinsic to the MFCs or may be due to the spatial resolution of the SOT/SP telescope and the typical size of the magnetic structures within plage. As the resolution element of the Hinode telescope is finite the field strength for narrower flux tubes will be systematically underestimated. Since the flux tubes comprising a single plage feature expand with height, neighbouring tubes merge to create a more homogenous feature leading to the narrower magnetic field strength distribution observed for core pixels at $\log (\tau)=-2.3$. The merging between individual plage features to form larger continuous regions of magnetic field can be seen in a myriad of locations in Figs. 3.2 \& 3.3. However, a change in the distance between neighbouring MFCs can lead to inhomogeneous magnetic field strength also above the merging height of the field (Bruls and Solanki 1995).

The distribution of the canopy pixels indicated by the dashed line in Fig. 3.5 reveals that $\mathrm{B}$ in the canopy is generally much weaker than in the core pixels. The distribution also has no obvious cut-off save for the arbitrary $300 G$ threshold, suggesting that the MFCs keep expanding with height in directions in which they are not hindered by neighbouring magnetic features. 


\subsubsection{Velocities}

Figures 3.6a-c show the LOS velocities retrieved by the inversion at the three nodes in $\log (\tau)$. The location of core pixels in the images are encompassed by the black contour line. Outside the areas harbouring strong magnetic fields, the typical quiet Sun granular convection pattern can be seen at $\log (\tau)=0 \&-0.9$. The LOS velocities in the top node outline only traces of the stronger granules and display some similarities with chromospheric observations, albeit with smaller velocity amplitudes.

Figures $3.6 \mathrm{a}-\mathrm{c}$ qualitatively indicate that the plasma in the majority of core pixels is nearly at rest in all $\log (\tau)$ nodes. The lower two $\log (\tau)$ nodes show that almost all the plages are surrounded by a lane of downflows. This lane is so common in fact that many magnetic features in Fig. 3.6 can be spotted based on this ring of downflows alone. The downflows are clearly located at the edges of the MFCs as can be deduced from the blow-up of a small part of the FOV plotted in Fig. 3.7. The downflows surrounding MFCs are often found outside the area of strong magnetic fields, but, as Fig. 3.7 shows, some of the downflows seem to be found within the magnetic elements, although all core pixels harbouring strong downflows are located at the edge of their respective MFC. Hence, it is likely that the narrow downflow lanes surrounding plages are not resolved properly. Therefore, the downflows appear to be coinciding with strong magnetic fields, but are in fact separated from each other. This result confirms the general lack of strong downflows found in plage by Solanki (1986) and Martínez Pillet et al. (1997). At no point was a strong downflow found at the centre of a MFC, which might have indicated at convective collapse taking place.

A more quantitive picture of the LOS velocities in core pixels can be gained through the use of histograms. Such histograms are plotted in Fig. 3.8, where the colour code is identical to the one used in Fig 3.5. It follows from this figure that the plasma in the majority of core pixels is nearly at rest. According to the histograms corresponding to $\log (\tau)=0 \&$ -0.9 some core pixels harbour strong downflows, which in isolated cases can reach LOS velocities of up to $8 \mathrm{~km} / \mathrm{s}$ at $\log (\tau)=0$. The majority of these large downflows, however, take values of $1-3 \mathrm{~km} / \mathrm{s}$, supporting the observation made by Langangen et al. (2007). But recall that these downflows are located at the edges of the MFCs. The downflows observed in the lower two $\log (\tau)$ nodes give the LOS velocity distributions an asymmetric appearance, which is supported quantitatively by the respective mean and median values of the distributions. At $\log (\tau)=0$ the mean velocity is $0.94 \mathrm{~km} / \mathrm{s}$ with a median of $0.63 \mathrm{~km} / \mathrm{s}$, whereas at $\log (\tau)=-0.9$ the mean and median velocities are $0.37 \mathrm{~km} / \mathrm{s}$ and $0.27 \mathrm{~km} / \mathrm{s}$ respectively. The los velocity distributions of both the core and canopy pixels at $\log (\tau)=-2.3$ are very symmetric and no longer show the strong downflows observed at the other two nodes. The mean and median velocities are almost identical at $0.17 \mathrm{~km} / \mathrm{s}$ and $0.15 \mathrm{~km} / \mathrm{s}$. This reduction supports the scenario that the downflows seen at the edges of MFCs are due to the convection of the plasma surrounding the magnetic element. Some of the core and canopy pixels show upflows of up to $1 \mathrm{~km} / \mathrm{s}$, which were also observed by Langangen et al. (2007). They are in most cases found within the MFCs and could be the result of oscillations or other transient events occurring within MFCs. Weak downflows of this order are also found in the bodies of the MFCs.

Figures 3.6 d-f show the inclination the magnetic field in the observer's frame of reference. The plage display rather vertical orientations (blue), but a closer inspection of the images 


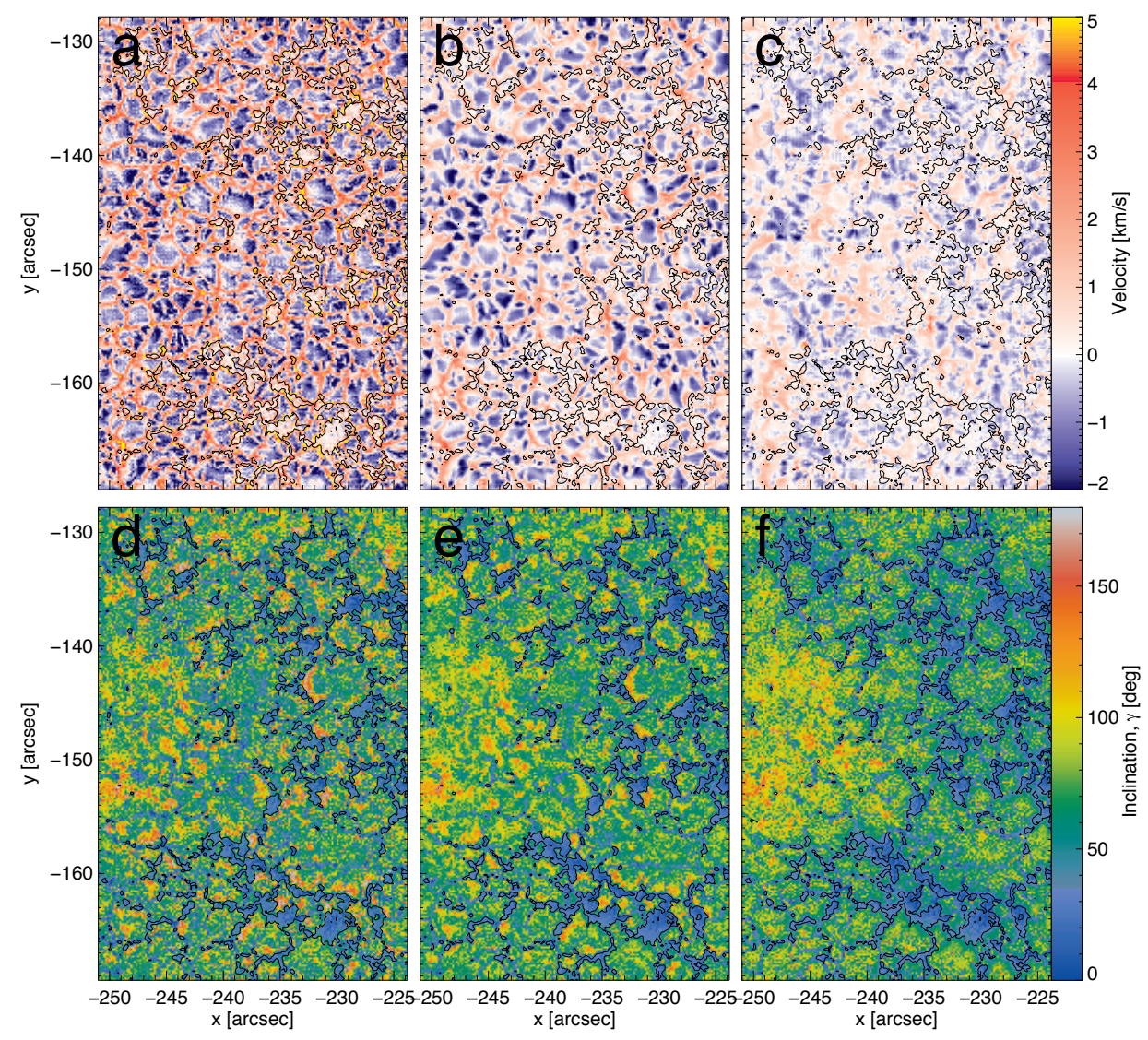

Figure 3.6: $a-c$ : The line-of-sight (LOS) velocities retrieved by the inversion at $\log (\tau)=$ $0,-0.9$ and -2.3 , from left to right. The velocity range is identical in all the three images. $d-f:$ The inclination of the magnetic field relative to the LOS, $\gamma$, obtained by the inversion at $\log (\tau)=0,-0.9$ and -2.3 , from left to right. All the three images share the same colour bar on the right. The black contours encompass pixels where $B>900 G$ at $\log (\tau)=0$ in all images.

corresponding the the $\log (\tau)=0 \&-0.9$ nodes reveals that many MFCs are adjoint by small patches with an inclination opposite to the MFC, red colour. Further examination of Figures 3.6d-f reveal that these small opposite polarity patches are hidden beneath the canopy of the main MFC with which they are associated. A typical example of such a patch can also be seen in more detail at $-231 X,-153 Y$ in Fig. 3.7. Field strengths of the vast majority of these small opposite polarity patches have $B<100 G$ at $\log (\tau)=0$, whereas the canopy above them has a field strength of at least $300 G$. This means that the Stokes spectra of these pixels are dominated by the canopy. Only the deconvolved Stokes profiles returned by the inversion, as displayed in Fig. 3.10, show a small polarity reversal in the wings of the Stokes $V$ profile.

Nonetheless a few pixels in these small opposite polarity patches have $B>100 G$ at $\log (\tau)=0$. These pixel were analyzed further by producing histograms of their los velocities. The histograms are depicted in Fig. 3.9 where only the distributions of the lower two $\log (\tau)$ nodes are shown, since the upper node no longer contains the reversed polarity. Both the histograms in Fig. 3.9 demonstrate that these pixels are predominantly 

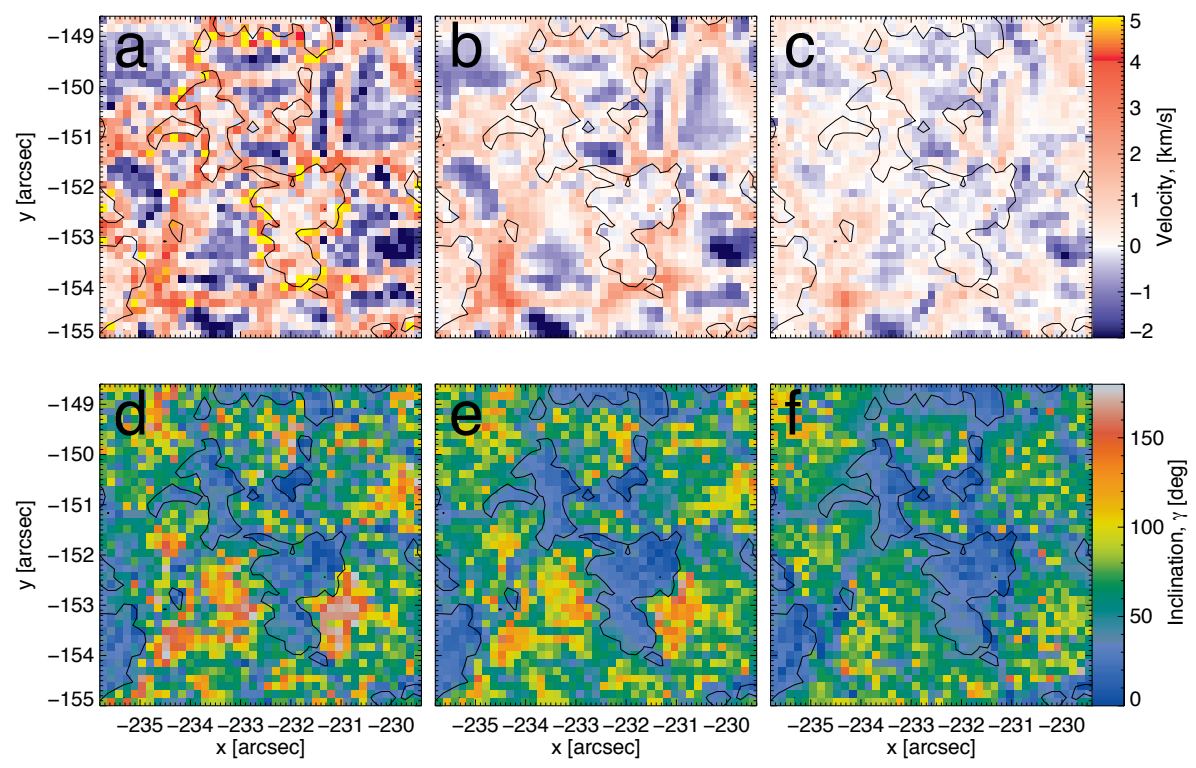

Figure 3.7: The same as Fig. 3.6, but for a small part of the full FOV, chosen to reveal the detailed spatial distribution of the LOS velocity and inclination in and around MFCs.

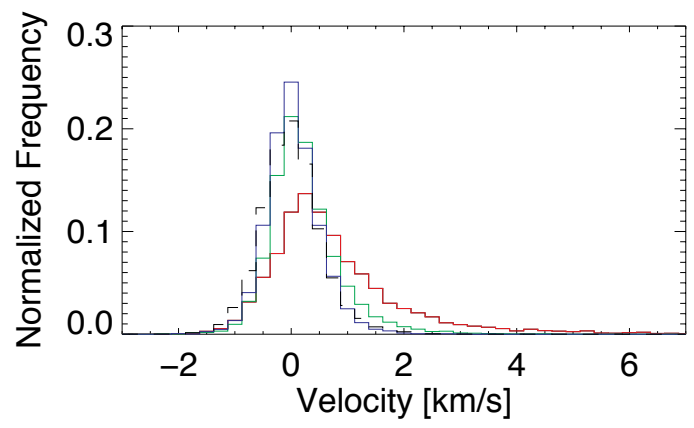

Figure 3.8: Histograms of the LOS velocities found in plages. The three coloured histograms were obtained using core pixels, where red refers to $\log (\tau)=0$, green to $\log (\tau)=-0.9$ and blue refers to $\log (\tau)=-2.3$. The dashed histogram shows the los velocities of canopy pixels at $\log (\tau)=-2.3$.

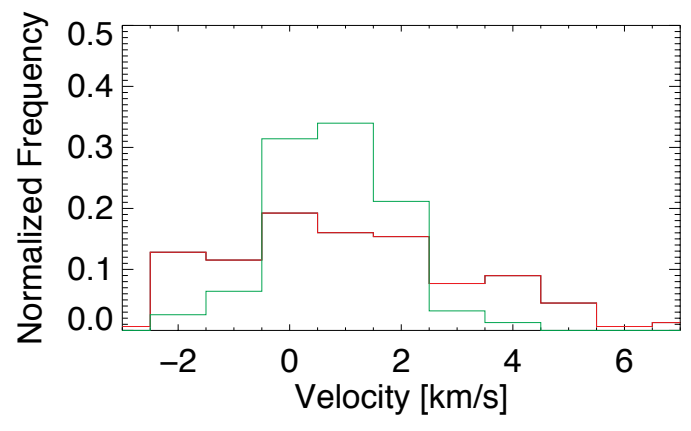

Figure 3.9: Histograms of the $\operatorname{los}$ velocities at $\log (\tau)=0 \&-2.3$, shown in red and green respectively, of the small opposite polarity patches adjoining plages features, seen in red in the lower three panels in Fig. 3.6. The histograms refer to those pixels that have $B>100 G$ at $\log (\tau)=0$. 


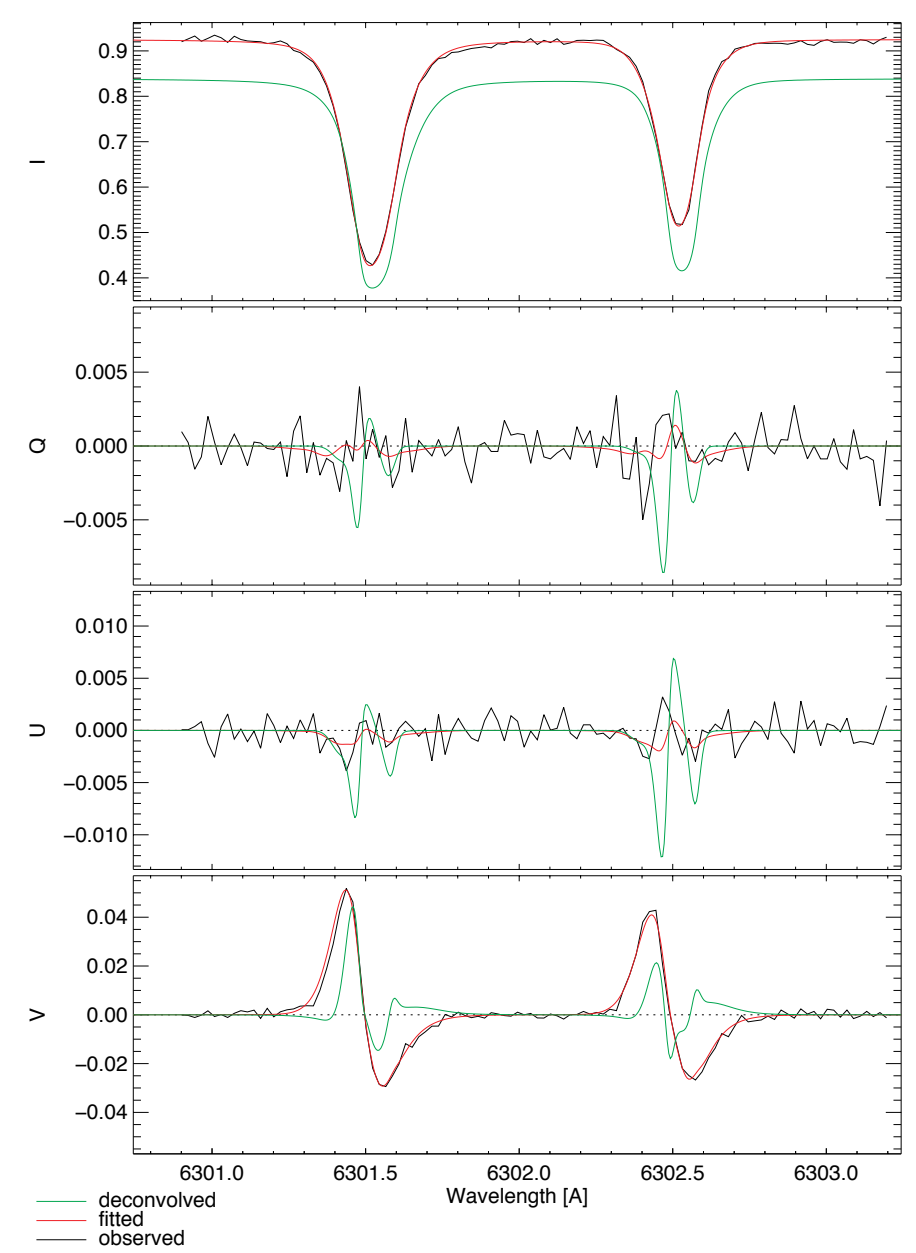

Figure 3.10: Stokes spectra of a typical canopy pixel harbouring a small opposite polarity magnetic field at $\log (\tau)=0$ and -0.9 . The black spectra correspond to the original SOT/SP observation, the red displays the spectra fitted by the inversion, and green shows the resultant spectra after the spatial deconvolution.

located in downflows, which surround the plage features. The mean and median velocities of these pixels at $\log (\tau)=0$ are $1.55 \mathrm{~km} / \mathrm{s}$ and $1.29 \mathrm{~km} / \mathrm{s}$ respectively. At $\log (\tau)=-0.9$ the mean and median velocities are $1.26 \mathrm{~km} / \mathrm{s}$ and $1.22 \mathrm{~km} / \mathrm{s}$. The magnetic field strength of the pixels is found to decrease with height essentially creating a field free region between the small opposite polarity patch and the canopy of the main plage feature. Such small opposite polarity features have been observed in magneto-hydrodynamic simulation of flux sheets immersed in a convecting medium (Steiner et al. 1998; Vögler et al. 2005), and indirect evidence was found in a network patch (Zayer et al.1989), although the spatial proximity to the MFC could not be established in that publication.

The weak magnetic fields found in the opposite polarity patches and the complete absence of any polarity reversal in the original observed Stokes spectra suggest that observations performed at a higher spatial resolution may be necessary to conclusively prove the existence of these patches. However, the similarity with the results of 3-D MHD simulations strongly supports our finding. 

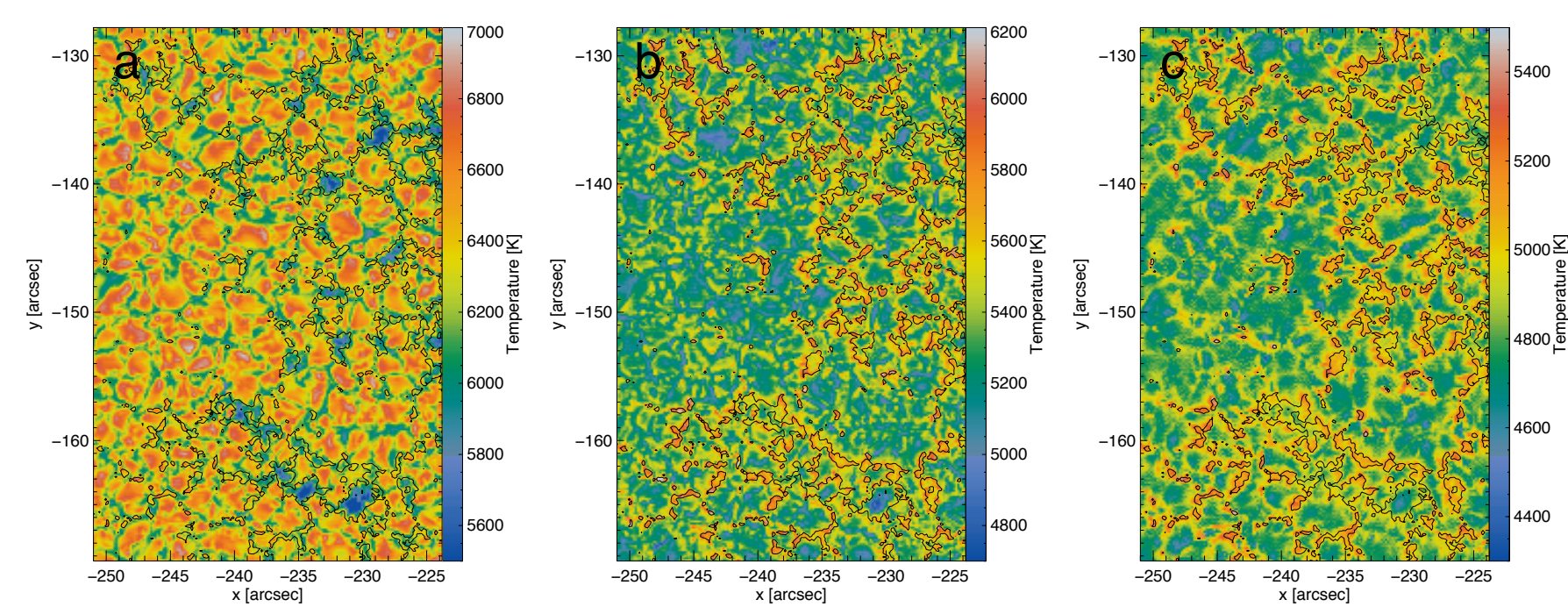

Figure 3.11: $a-c$ : Temperature at $\log (\tau)=0,-0.9$ and -2.3 , respectively. The black contour lines encompass core pixels where $B=900 G$ at $\log (\tau)=0$.

\subsubsection{Temperature}

The inversion retrieved a temperature stratification for each pixel of the analyzed region. The temperatures at each of the three $\log (\tau)$ nodes is displayed in Fig. 3.11 for the same FOV as in Fig. 3.6. Fig. 3.11 a corresponds to the temperature at $\log (\tau)=0$ and exhibits the familiar granulation pattern. The positions of core pixels are revealed by the black contour line in the image and show that they are predominantly found within the comparatively cool intergranular lanes. Several pores and micropores of various sizes can also be identified in this image based on their low temperatures. The largest of these pores is situated at $-230 X$, $-165 Y$. Figures $3.11 \mathrm{~b} \& 3.11 \mathrm{c}$ display the temperatures found at $\log (\tau)=-0.9$ and -2.3 , respectively. Both images indicate the comparatively high temperatures found within MFCs when compared to the quiet Sun at these heights. The temperatures at $\log (\tau)=-2.3$ furthermore not only reveal the comparatively hot MFCs but also a reversed granulation pattern. Although pores no longer are cooler than the quiet $\operatorname{Sun}$ at $\log (\tau)=2.3$, they do remain less hot than the smaller MFCs.

The higher temperatures within MFCs at $\log (\tau)=-0.9$ and -2.3 , when compared to the quiet Sun, are also illustrated by the histograms in Fig. 3.12. At both those layers the average temperature is around $300 \mathrm{~K}$ higher within core pixels, with average temperatures of $5690 \mathrm{~K}$ and $5070 \mathrm{~K}$ at $\log (\tau)=-0.9$ and -2.3 respectively, than in quiet Sun pixels where the average temperatures at the same $\log (\tau)$ heights are $5290 \mathrm{~K}$ and $4780 \mathrm{~K}$. Fig. 3.12 . also demonstrates that the average temperature in the canopy pixels at $\log (\tau)=-2.3$, taking a mean value of $5000 \mathrm{~K}$, is similar to the temperatures of core pixels at the same height. Only at $\log (\tau)=0$ is the average temperature higher in the quiet Sun, at $6410 \mathrm{~K}$, than in the core pixels, which have a mean temperature of $6270 \mathrm{~K}$. The temperature of quiet Sun pixels located in downflowing regions, dash-dot-dot-dot histogram in Fig. 3.12 , have a slightly lower mean temperature at $6240 \mathrm{~K}$ than MFCs, which are also located predominantly in downflowing regions. The cool tail of the MFCs is populated by pores. The temperatures of quiet Sun pixels in upflowing regions, dash-dot histogram in Fig. 

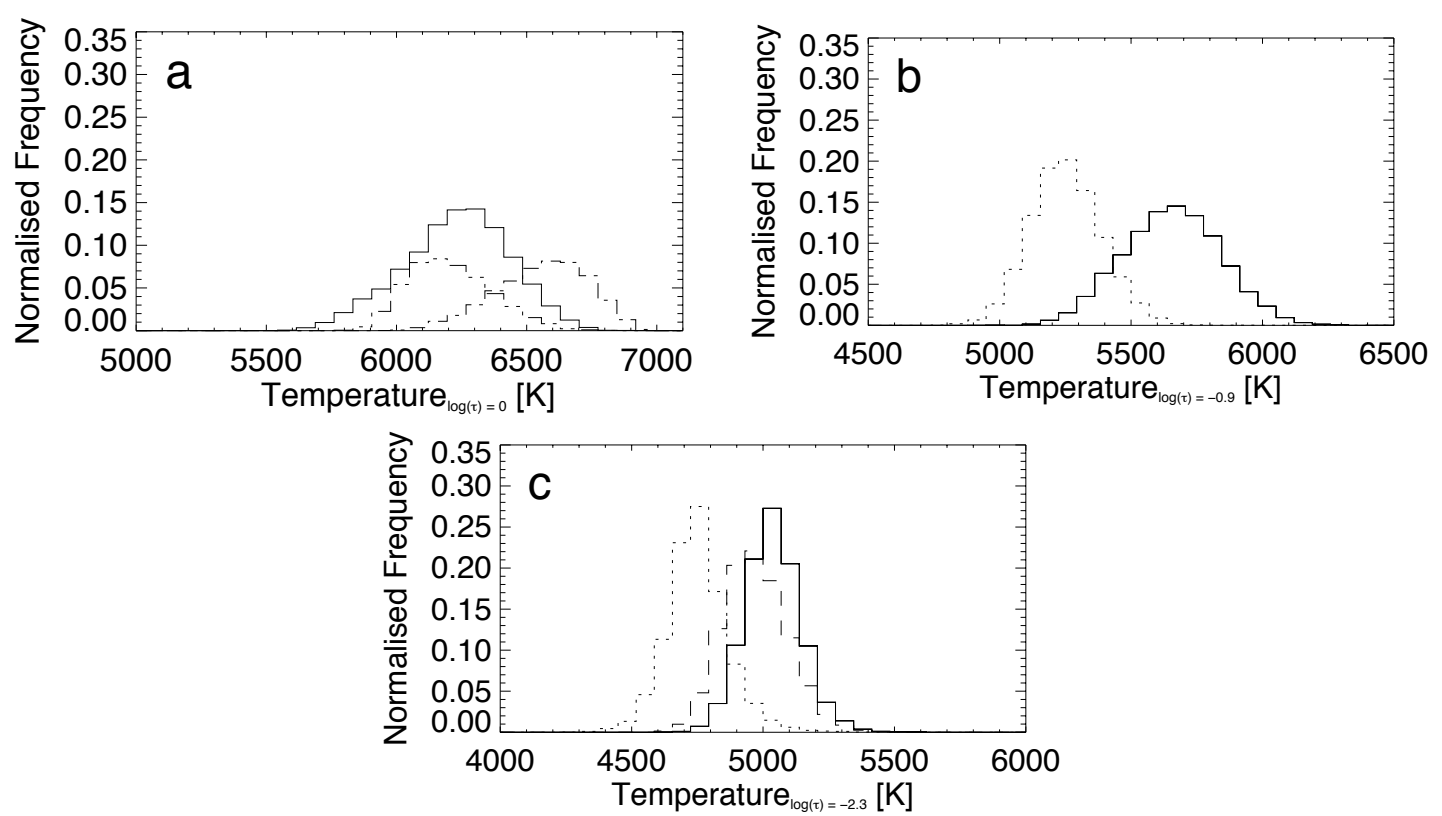

Figure 3.12: $a$ : Temperature histograms of core plage pixels, solid line, and the quiet Sun at $\log (\tau)=0$. The quiet Sun temperatures have been further divided into temperature histograms corresponding to downflowing, dash-dot-dot-dot, and upflowing, dash-dot, regions. $b$ : Temperature histograms of core plage pixels, solid, and the quiet Sun, dotted, at $\log (\tau)=-0.9$. $c$ : Temperature histograms of core plage pixels, solid, and the quiet Sun, dotted, at $\log (\tau)=-2.3$. The dashed histogram displays the temperatures in canopy pixels.

3.12 a, have a mean temperature of $6590 \mathrm{~K}$.

The temperature stratification within core pixels was studied further, first, by taking the ratio of the $\log (\tau)=0$ and -2.3 temperatures. A histogram of these ratios is seen in the left panel of Fig. 3.13. The average ratio for core pixels is $0.81 \pm 0.02$, which demonstrates that the majority of core pixels have a very similar temperature stratification. The ratios were then compared to the temperature ratio obtained from a plage flux tube model derived by Solanki and Brigljevic (1992), which is shown by the dotted line in the left panel in Fig. 3.13. The temperature ratio of the model, which has a ratio of 0.79 , agrees reasonably well with the inversion results. A comparison between simulation results and the plage flux tube model was carried out by Vitas et al. (2009), who found a good agreement. An alternative thin flux tube model, aimed at modeling flux tubes in the network described by Solanki (1986), has a ratio of 0.7 between the same $\log (\tau)$ heights. The temperature stratification of MFCs studied in this investigation significantly deviate from the network model's prediction.

The temperature stratification of the plage model of Solanki and Brigljevic (1992) along a finer $\log (\tau)$ grid is shown by the solid line in the right panel of Fig. 3.13 . The dotted line in the same panel shows the typical temperature stratification obtained from core pixels by the inversion. Whilst quantitatively the model and inversion result agree quite well in particular at the three $\log (\tau)$ nodes, qualitatively there is an important difference between the model and inversion result. The temperature stratification of the model has a notable bend between $\log (\tau)=-1$ and -1.5 , which is entirely absent from the inversion result. 

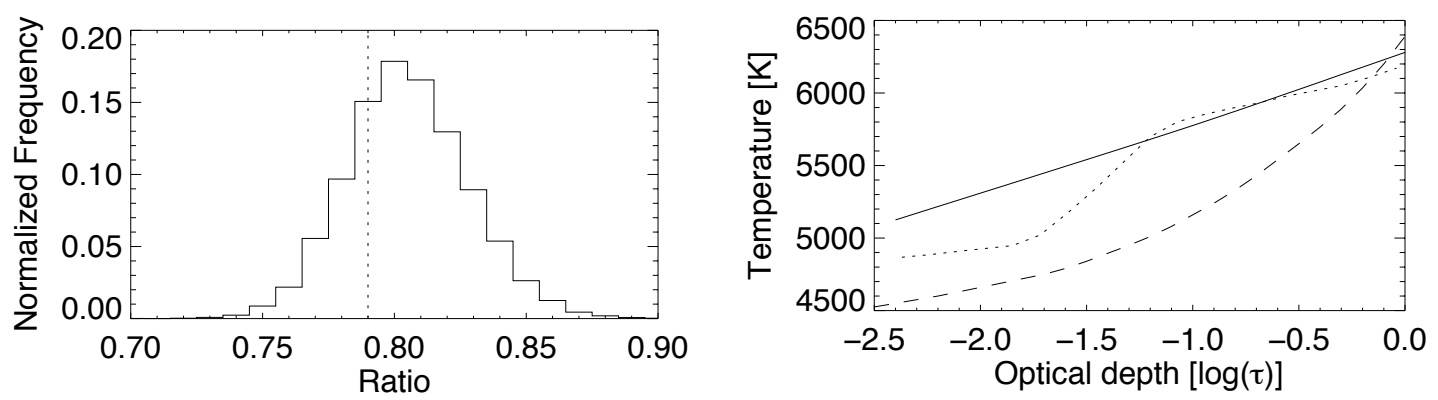

Figure 3.13: Left: Distribution of temperature ratios of the $\log (\tau)=0$ and -2.3 layers of all core pixels. The dotted line shows the same ratio obtained from the plage flux tube model of Solanki and Brigljevic (1992). Right: The solid line shows the temperature stratification of a typical core pixel obtained from the inversion. The dotted line follows the the temperature stratification of an ideal plage flux tube model. The dashed line depicts the temperature stratification of the HSRA.

However, such a bend can only potentially be reproduced by the inversion by employing at least four nodes, which would in turn introduce extra free parameters.

\subsubsection{Inclination \& Azimuth}

The inclinations of the magnetic field plotted in Figs. 3.2 and 3.6 are inclinations in the observer's frame of reference. Due to the small heliocentric angle $\left(\langle\theta\rangle=13^{\circ}\right)$ a qualitative picture of the inclinations of the various plage regions could still be gained from those figures. However, a conversion of these inclinations to local solar coordinates is necessary to find the inclination of the magnetic fields with respect to the solar surface.

The conversion of the inclinations and azimuths retrieved by the inversion to local solar coordinates is not straightforward. Whilst the inclination of the magnetic field is uniquely defined the azimuth on the other hand has an inherent $180^{\circ}$ ambiguity (Unno 1956). Therefore, when converting to local solar coordinates one is forced to choose between one of two possible solutions for the magnetic field vector.

Many codes, requiring various amounts of manual input, have been developed to solve the $180^{\circ}$ ambiguity, the reader is directed to Metcalf et al. (2006) and Leka et al. (2009) for an overview. An additional challenge facing these codes is that they generally use the output of an ME inversion as an input. The output of the ME inversion does not contain any information on the change of the magnetic field vector over the formation height of the inverted absorption line. The SPINOR code, however, provides this information, which in turn allows the canopies of magnetic features to be identified as is shown in Fig. 3.3. The azimuths retrieved by the inversion were spatially very smooth in all the nodes, indicating that the azimuth was well defined in the regions containing magnetic fields.

The canopy pixels shown in Fig. 3.3 form continuous rings around the various cores. By assuming that the magnetic field in each canopy pixel originates from the largest patch of core pixels in its immediate surrounding, the direction of the magnetic field vector of each canopy pixel could be determined unambiguously as long as the polarity of the corresponding core patch was known. The polarity of a patch of core pixels could be determine easily from their Stokes $V$ spectra. In essence, the magnetic field vector of 
a canopy pixel would point towards a patch of core pixels if it had a negative polarity and, in turn, away from it if the patch of core pixels had a positive polarity. This process was repeated for each canopy pixel individually. Since the canopies of the various plage features are greatest at $\log (\tau)=-2.3$, the magnetic field vector of the canopy pixels was determined at this $\log (\tau)$ height.

Once the magnetic field vectors of the canopy pixels were determined, the vectors of the core could be determined using an acute-angle method. The acute-angle works by performing dot products, using the two possible vectors in an undetermined pixel, with those surrounding pixels whose vector was already determined. The dot products corresponding to each of the two possible solution were then summed. The vector associated with the smallest sum was subsequently selected as the correct vector for that pixel. The pixels that were surrounded by the largest number of determined vectors were given preference.

Now that the magnetic field vectors of both the canopy and core pixels at $\log (\tau)=-2.3$ are known, the vectors at $\log (\tau)=0 \&-0.9$ could also be determined. The now known vector at $\log (\tau)=-2.3$ in each pixels was used to perform a dot products with the two possible vectors in the next lower $\log (\tau)$ layer. The vector with the smaller dot product was subsequently chosen as the correct magnetic field vector. The $180^{\circ}$ ambiguity of the magnetic field vector at $\log (\tau)=0 \&-0.9$ was removed for only those pixels where $B>700 G$ in either layer. The resolution of all the vectors is entirely automatic and only the definition of the core and canopy pixels for the initial input is manual, but followed the definition given in Sect. 3. Also, no smoothing of the azimuths is performed at any point. The converted inclinations and azimuths in local solar coordinates after the resolution of the $180^{\circ}$ ambiguity are plotted in Fig. 3.14

Figures $3.14 \mathrm{a}-\mathrm{c}$ show the resolved azimuths, $\varphi$, at all three nodes and several azimuth 'centres' (Martínez Pillet et al. 1997) can be readily identified, best at $\log (\tau)=-2.3$. In combination with Figures $3.14 \mathrm{~d}-\mathrm{f}$ it can be seen that most of the azimuth 'centres' have relatively vertical fields in their cores that become more horizontal towards the edges of a magnetic feature. This picture is consistent with a magnetic feature expanding with height in all directions. Such azimuth centers tend to be either relatively isolated features or large ones. Most of the magnetic structures tend to be elongated, but show similar structure, with the field expanding roughly perpendicular away from the long axis of the structure over most of its length and directed radially away at the ends.

A more quantitive picture of the general inclinations of the core pixels can be obtained through histograms, which are depicted in Fig. 3.15. The distributions of the inclinations have their peak between $10^{\circ}$ and $15^{\circ}$ for all $\log (\tau)$ nodes. This confirms earlier observations by Topka et al. (1992); Bernasconi et al. (1995) and Martínez Pillet et al. (1997), who found similar peak inclinations for plage. The mean inclination for each $\log (\tau)$ layer is $21.9^{\circ}, 18.4^{\circ}$ and $23.0^{\circ}$ with decreasing optical depth. The median values have a similar progression with optical depth taking values of $18.9^{\circ}, 16.7^{\circ}$ and $21.4^{\circ}$ respectively. Fig. 3.15 also shows that the canopy pixels are significantly more horizontal with a peak at $25^{\circ}$ and a very extended tail reaching to $90^{\circ}$. The mean inclination for the canopy fields is $39.1^{\circ}$ with a median of $36.4^{\circ}$, which demonstrates quantitatively the more horizontal nature of the canopy when compared to the core fields. The largest inclinations are found at the edges of the canopies as expected for an expanding flux tube or flux sheet.

Fig. 3.16 shows the histograms of $\varphi$ of core pixels. None of the four distributions are homogeneous and show a consistent under-representation of the $W$ and partly the $N$ 


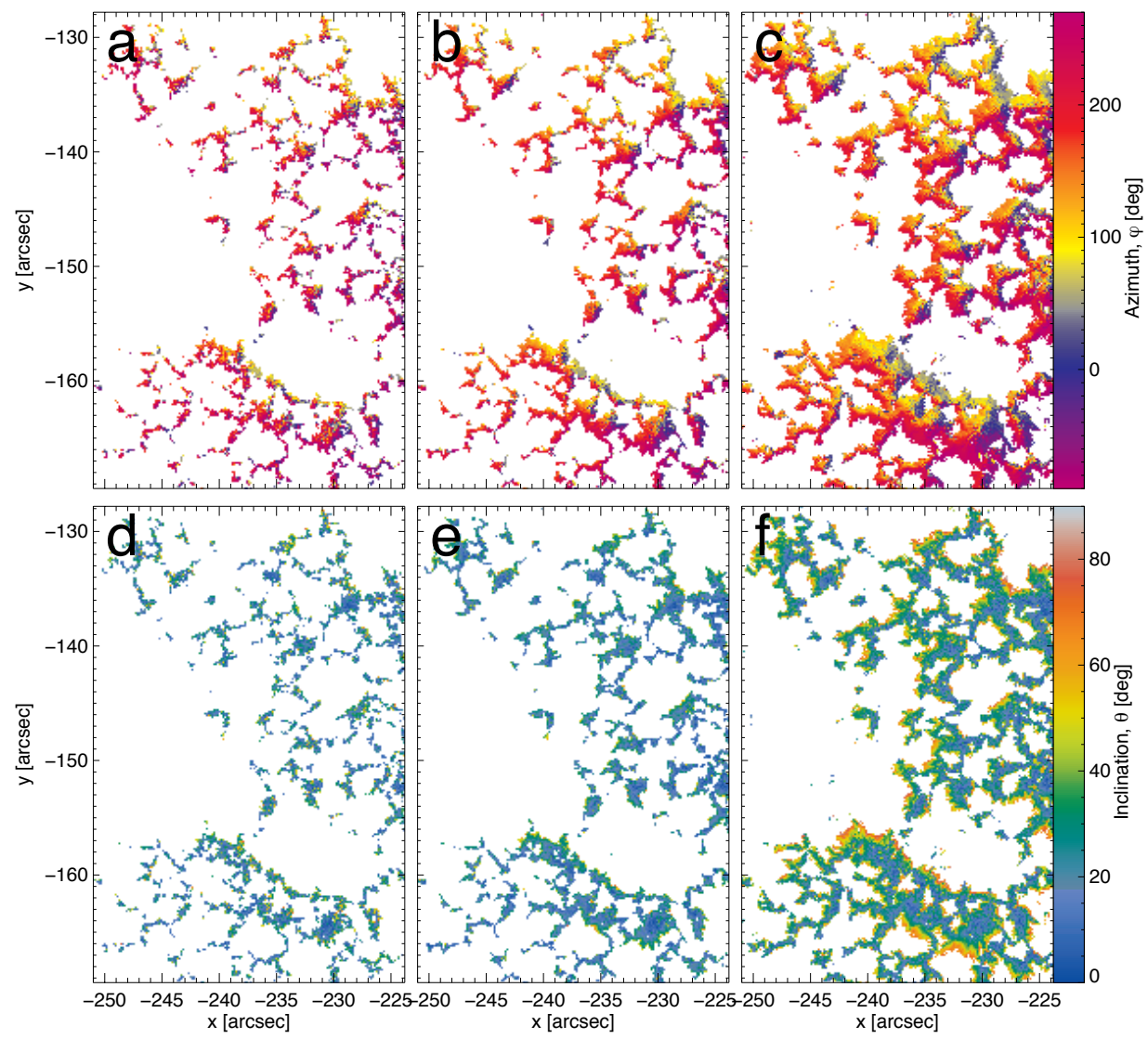

Figure 3.14: $a-c$ : The ambiguity resolved azimuths in local solar coordinates, $\varphi$, at $\log (\tau)=0,-0.9$ and -2.3 from left to right. North is up and corresponds to an angle of $90^{\circ}$. West is to the right and corresponds to an angle of $0^{\circ} . d-f$ : The local solar coordinate corrected inclinations, $\theta$, of the magnetic field after the azimuth ambiguity resolution at $\log (\tau)=0,-0.9$ and -2.3 from left to right. The inclinations and azimuths of pixels with $B<300 G$ at $\log (\tau)=-2.3$ and $B<700 G$ at $\log (\tau)=0 \&-0.9$ are shown in white.

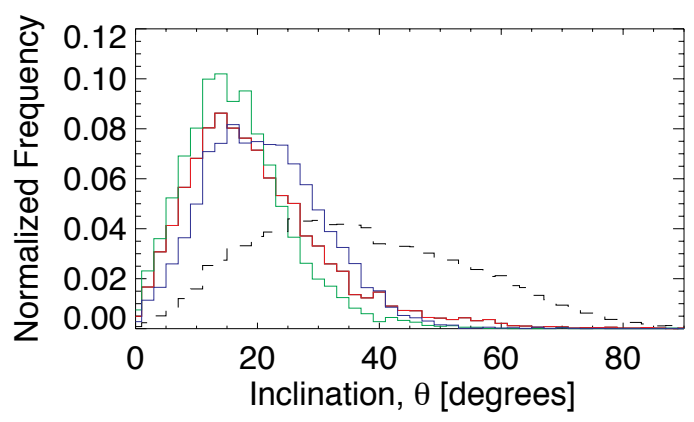

Figure 3.15: Histograms of magnetic field inclination of MFCs relative to the solar surface normal, $\theta$. The three coloured histograms were obtained using core pixels, where red refers to $\log (\tau)=0$, green shows to $\log (\tau)=-0.9$ and blue refers to $\log (\tau)=-2.3$. The dashed histogram depicts $\theta$ of canopy pixels at $\log (\tau)=-2.3$. 


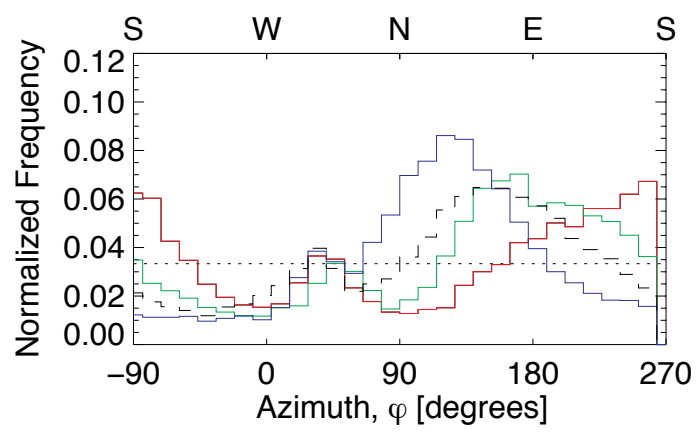

Figure 3.16: Histograms of $\varphi$ found in plages. The three coloured histograms were obtained using core pixels, where red refers to $\log (\tau)=0$, green shows to $\log (\tau)=-0.9$ and blue refers to $\log (\tau)=-2.3$. The dashed histogram shows $\varphi$ of canopy pixels at $\log (\tau)=-2.3$. The dotted line represents a homogeneous distribution.

directions. These two directions are, however, expected to be under-represented due to the viewing geometry, as the region was located in the $S E$ at the time of the observation. The azimuth distributions from plages found at the northern edge of the field of view show a more homogeneous distribution, as expected. This supports the conclusion drawn by Martínez Pillet et al. (1997), who found no preferred azimuth direction for plages. On the other hand, Bernasconi et al. (1995) did find a preferred $E-W$ direction for plages.

Fig. 3.16 also shows that the peak of each azimuth distribution is shifted with respected to other distributions, suggesting that the direction of the magnetic field vector of individual pixels in plages appears to rotate or twist. Although a solar origin of the twist cannot be ruled out, it can very easily be an artifact of the inversion. A cross talk between the Stokes parameters of $1-2 \%$ from either Stokes $I$ or $V$ into Stokes $Q$ and/or $U$ could reproduce the typical azimuth rotations returned by the inversion. An error in the line damping parameters employed by inversion could also be the culprit. Therefore, we do not attach any importance to this result.

\subsubsection{Magnetic field gradient}

The change in the peak magnetic field strength in each of the coloured histograms in Fig. 3.5 indicates that the magnetic field strength of the core pixels decreases with height. This was investigated further, first, by correlating the relative decrease, $d$, of the magnetic field for each core pixel using,

$$
d=\frac{B(\log (\tau)=-2.3)}{B(\log (\tau)=0)},
$$

against $B_{z}(\log (\tau)=0)$, which is shown in Fig. 3.17. The dashed line in Fig. 3.17 shows the $d$ predicted by a thin flux model with $B_{z}=2000 G$ at $\log (\tau)=0$. The thin flux model is identical to the plage model described in Solanki and Brigljevic (1992).

Fig. 3.17 shows that only magnetic fields above $2000 G$ have a $d$ closer to the thin flux tube model, than weaker fields. The reduced gradient in $B$ for these weak fields is likely to stem from our definition of core pixels. The core pixels with weakest B are found at the edge of their respective MFC and thus may already be partially part of the canopy, due to 


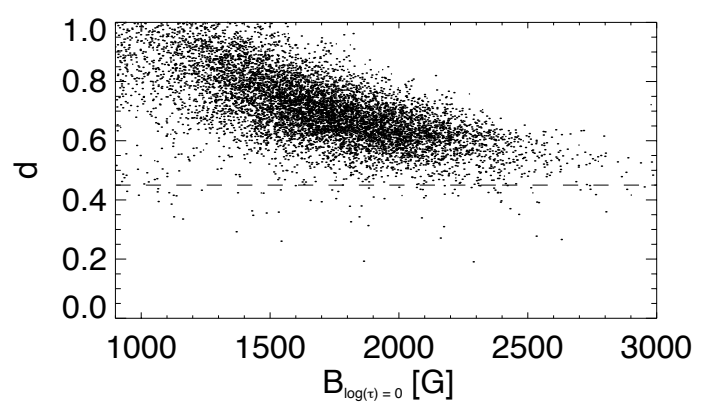

Figure 3.17: The ratio between field strengths at different $\log (\tau), d$, calculated using Eq. 3, of core pixels plotted against the local solar coordinate corrected LOS magnetic field, $B$. The dashed line indicates the $d$ of a thin flux tube with $B=2000 G$ at $\log (\tau)=0$.

the limited spatial resolution. This would reduce the vertical field strength gradient, thus increasing $d$. Furthermore, the small opposite polarity patches described in Sect. 4.2 could reach right up to the edge of an MFC, allowing the possibility of two opposite polarity magnetic fields to exist within a weak core pixel. The Stokes $V$ signals from those two fields would at least partially cancel each other, leading to a reduction in the retrieved $B$ value in the lower two $\log (\tau)$ nodes and hence to an increase in $d$. Another possibility is that the weak core pixels contain flux tubes which are not fully resolved by Hinode, in particular in the lower two layers. The expansion of such an unresolved flux tube would then take place primarily within the pixel, leading to a nearly unchanging $B$ in all three $\log (\tau)$ nodes, i.e. a $d$ close to unity. Even stronger fields display a $d$ that is larger than the thin tube value. Partly this has to do with the dense clustering of magnetic features in many parts of the investigated plage, which does not allow the field to expand freely, thus also reducing the vertical gradient of the field strength. Also, the gradient we derive is likely a lower limit of the true gradient (see Sect. 4.6). We also draw attention to the fact that the strongest fields in the inverted region belong to the small pores abundantly present there (see Sect. 4.3). These pores are sufficiently large that their magnetic stratifications are unlikely to follow the thin-tube approximation.

The inversion also returns a geometric scale for each pixel. However, the inversion process only prescribes hydrostatic equilibrium within each pixel but, does not impose horizontal pressure balance, in particular across pixels. Therefore, each pixel has an individual geometric height scale, which can be off-set with respect to other pixels. This makes the comparison of gradients in (e.g.) $B$ between pixels with very different atmospheres difficult. However, the core pixels found at the very centre of a plage feature, with $B<2000 G$ from Fig. 3.17, have very similar atmospheres to each other, allowing the estimation of a common gradient in $B$. The gradient, $\Delta B$, in the magnetic field of these core pixels is $-2.6 \pm 0.5 G / \mathrm{km}$ between $\log (\tau)=0$ and $\log (\tau)=-2.3$. This gradient is consistent with the gradient calculated by Martínez Pillet et al. (1997) of $\Delta B=-3 \pm 1 G / \mathrm{km}$ within one $\sigma$. The $\Delta B$ from the inversion is also smaller than the gradient given by the thin flux tube model, which takes a value of $\Delta B=-3.9 \mathrm{G} / \mathrm{km}$ over the same interval in $\log (\tau)$. This discrepancy can be partly explained by the different magnetic field values at $\log (\tau)=0$ between the model and the observation. 


\subsubsection{Expansion of magnetic features with height}

Fig. 3.2 and, in particular, Fig. 3.3 demonstrate that the magnetic features seen in the field of view expand with height. Furthermore, Fig. 3.14 reveals that the magnetic features generally expand in all directions and are not subjected to extreme foreshortening effects or deformations, except due to other nearby MFCs. This raises the question of how close this expansion is to that of a model thin flux tube. Several methods were tested to find a robust measure of the change in size of a magnetic feature with height. The most obvious method, the conservation of magnetic flux with height, was found to be unreliable to estimate the expansion of the magnetic features. The LOS flux of a magnetic feature was seen to increase by about $20 \%$ with height when taking the 'visible' boundary as a rough guide. This in turn led to a systematic underestimation of the true size of a feature when magnetic flux conservation was taken as a criterion. This may partly be due to cancellation of some of the Stokes $V$ signal with that from the surrounding opposite polarity fields in lower layers. Therefore, the change in size of a magnetic feature was estimated using a method that was independent of magnetic field strength values returned by the inversion. The systematic overestimation of the magnetic field at $\log (\tau)=-2.3$ by around $20 \%$, when compared to the other two $\log (\tau)$ layers, also means that magnetic field gradient in the core pixels, $\Delta B=-2.6 \pm 0.5 \mathrm{G} / \mathrm{km}$, should be viewed as a lower limit only.

The expansion of the magnetic features was finally estimated the following way. At $\log (\tau)=0$ the size of a magnetic feature was arbitrarily defined by the number of pixels that harboured a magnetic field of at least $900 G$. Then all the magnetic field values in the $\log (\tau)=0$ image were normalized by the maximum field strength in the feature and the ratio, $r_{t}$, was calculated using $r_{t}=\frac{900 G}{B_{\max }(\tau=1)}$. Each subsequent $\log (\tau)$ layer above $\log (\tau)=0$ was in turn normalized by its own $B_{\max }(\tau)$ value. The expansion of a magnetic feature could then be tracked by the total number of pixels at a given $\log (\tau)$ layer where $\frac{\left.B_{(} \tau\right)}{B_{\max }(\tau)}>r_{t}$. Rather than track the expansion of a feature using only the three $\log (\tau)$ nodes returned by the inversion, the change of the magnetic field with height was tracked using a finer grid of $\log (\tau)$ layers, with a $\log (\tau)$ increment of 0.1 . This finer $\log (\tau)$ grid was created using the same spline interpolation between the three nodes as was used during the fitting by the inversion procedure. The $300 \mathrm{G}$ threshold used to select canopy pixel in other parts of this investigation was not imposed here in order to avoid an artificial obstruction of the expansion. Finally, the relative expansion of a feature was calculated using,

$$
\frac{R(\tau)}{R_{0}}=\sqrt{\frac{A(\tau)}{A_{0}}},
$$

where $R$ is the radius of the flux tube at optical depth $\tau, R_{0}$ is the radius of the flux tube at $\log (\tau)=0, A$ is the area of the fluxtube at optical depth $\tau$ and $A_{0}$ is the area of the flux tube at $\log (\tau)=0$.

Seven isolated magnetic features were selected from the field of view. The number of selected features is small since most magnetic features merge with other features at $\log (\tau)=-2.3$ as is demonstrated in Figs. $3.2 \& 3.3$. The relative expansions of the seven features that were selected are shown in Fig. 3.18. The solid line in Fig. 3.18 shows the relative expansion of the $0^{\text {th }}$ order thin flux tube plage model, described by Solanki and Brigljevic (1992). All the dotted lines in Fig. 3.18 corresponding to the seven selected magnetic features follow the expansion predicted by the plage model reasonably well. 


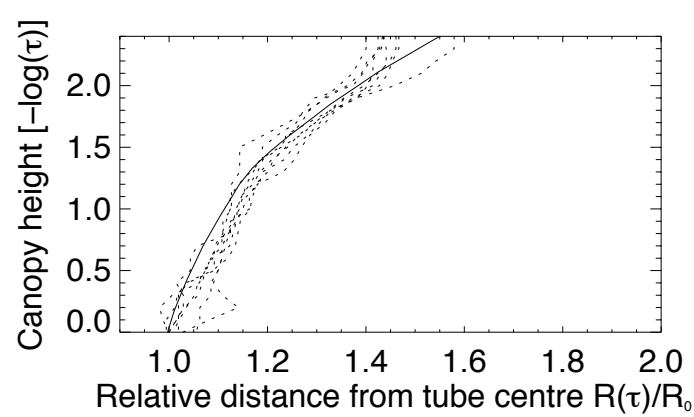

Figure 3.18: The dotted lines show the relative expansions of seven isolated magnetic features using Eq. 2. The solid line shows the relative expansion of a zeroth order thin flux tube model.

As was the case in Sect. 4.3 the thin flux tube model for the solar network did not fit the expansion of the observed MFCs as well as the plage model shown in Fig. 3.18. Our result supports the conclusion reached by Pietarila et al. (2010) that small, intense magnetic features in SOT/SP images can be modeled as thin flux tubes. The reduced relative expansion of the selected features above $\log (\tau)=-2$ when compared to the model may be an indication of the merging of features limiting the expansion at those heights. Another possibility is that a zeroth order model is not sufficient to describe the expansion of the selected features, especially in higher layers (Yelles Chaouche et al. 2009). The lateral variation of the magnetic field within the tube is no longer negligible in higher order flux tube models (Pneuman et al. 1986).

\subsubsection{Effect of the sunspot}

The majority of the MFCs show no obvious and conclusive influence of the sunspot's magnetic field, which stretches well beyond the spot's visible boundary in the form of a low-lying magnetic canopy (Giovanelli 1982; Solanki et al. 1992a, 1994). Therefore, they behave like isolated magnetic features, at least in the photosphere. There are, however, a few MFCs in the field of view where the influence of the sunspot can be seen in the photosphere. The most striking of these features is located at $-217 X,-125 Y$ in Figs. 3.2 $\&$ 3.3, where an extensive loop system can be identified. These loops have horizontal fields and connect several pores with positive polarity to the negative polarity sunspot. The magnetic field strengths found in this loop system can reach values as high as $1000 G$ in a few places. They confirm the existence of similar structures that were found by Lites and Skumanich (1990) and Martínez Pillet et al. (1997) when studying plage, but reveal also their fine-structure and show that they are suspended above the quiet Sun.

Plages close to the sunspot also posses highly deformed canopies, which are elongated towards the spot if the plages have the opposite polarity of the spot. Such plages can be seen at $-220 X,-170 Y$ and their inclinations and azimuths are shown in Fig. 3.19. The field in the core pixels of these MFCs is more inclined than average; compare with Fig. 3.15 . The mean inclinations of the field at the three layers from $\log (\tau)=0$ to $\log (\tau)=-2.3$ are $31.1^{\circ}, 32.3^{\circ}$ and $35.1^{\circ}$, respectively. These average inclinations are around $10^{\circ}$ larger than for MFCs found further away from the spot. It is in particular the inclinations of the canopy pixels in Fig. 3.19 that show the effect of the sunspot upon these magnetic fields. 

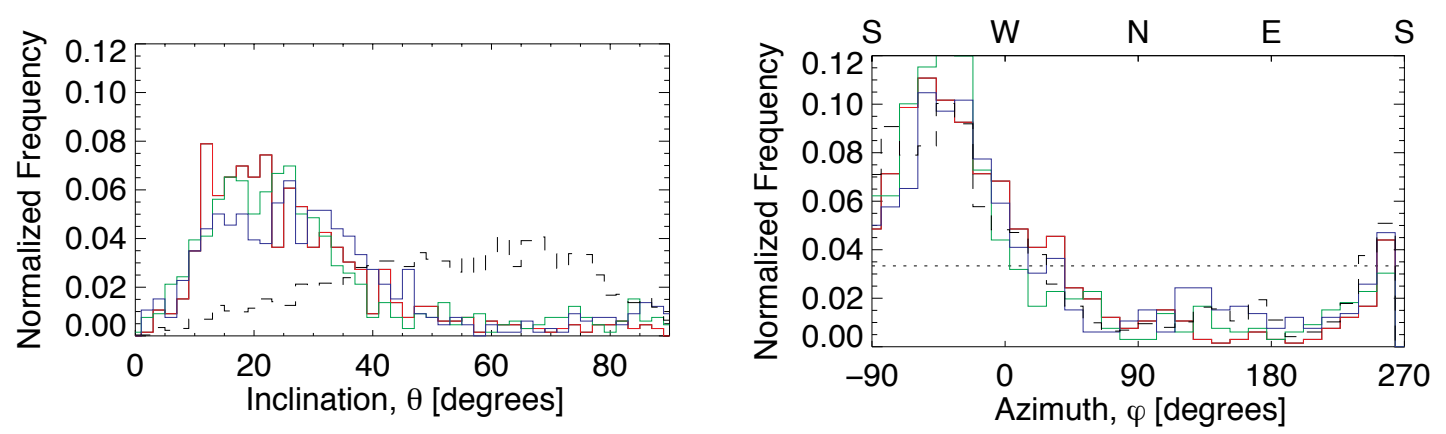

Figure 3.19: Left: Histograms of $\theta$ found in plage around $-220 X,-170 Y$. The three coloured histograms were obtained using core pixels, where red refers to $\log (\tau)=0$, green shows to $\log (\tau)=-0.9$ and blue refers to $\log (\tau)=-2.3$. The dashed histogram represents $\theta$ of canopy pixels at $\log (\tau)=-2.3$. Right: Histograms of $\varphi$ found in plages around $-220 X$, $-170 Y$. The three colours and the dashed line have the same significance as in the graph on the left in this figure. The dotted line represents a homogeneous distribution.

The mean inclination of the canopy fields is $55.8^{\circ}$ and the median value is $56.8^{\circ}$, which is more than $15^{\circ}$ larger than in Fig. 3.15. The azimuth distributions in Fig. 3.19 clearly display the influence of the sunspot as all the distributions both from the core and canopy pixels show a clear preferred orientation towards the spot. This inclination towards the spot is probably due to the connection of magnetic field lines starting in the MFC, part of their canopy becoming common with the sunspot's canopy and ending in the penumbra. The inclination may be exaggerated by the presence of the sunspot's canopy, especially if the MFCs are not fully spatially resolved (Solanki et al. 1996a).

From the examples given in this section the following picture emerges. Those magnetic features that are in the immediate vicinity of the sunspot, i.e. if there are no further $k G$ magnetic fields in between them and the spot, show a clear deformation of their canopy and have inclination and azimuth distributions that either predominantly point towards or away from the spot, depending on the polarity. The spot's influence on the orientation of magnetic features could be observed up to 20" away from the sunspot's outer penumbral boundary, provided there were no other magnetic features in between. Any magnetic feature situated behind this 'first row' of $k G$ features is effectively shielded from the spot in the photosphere and then behaves like an isolated magnetic feature, affected only by its nearest neighbours.

\subsubsection{Microturbulence}

Holweger et al. (1978) found that both macro- and height independent microturbulent velocities are required in order to fit profiles of photospheric spectral lines. Solanki (1986) also needed both, a height independent micro- and macroturbulence to fit the width of Stokes $V$ profiles of plage accurately. The total rms turbulent velocities had typical values of $1 \mathrm{~km} / \mathrm{s}$ to $3.5 \mathrm{~km} / \mathrm{s}$ in these fits, depending on the spectral line, in particular on line strength. Zayer et al. (1989) found, using lines in the infrared, that rms turbulent velocities between $3 \mathrm{~km} / \mathrm{s}$ and $3.5 \mathrm{~km} / \mathrm{s}$ were necessary to fit the observed profile shapes. The dependence on spectral line strength suggests a height dependent turbulent velocity. 


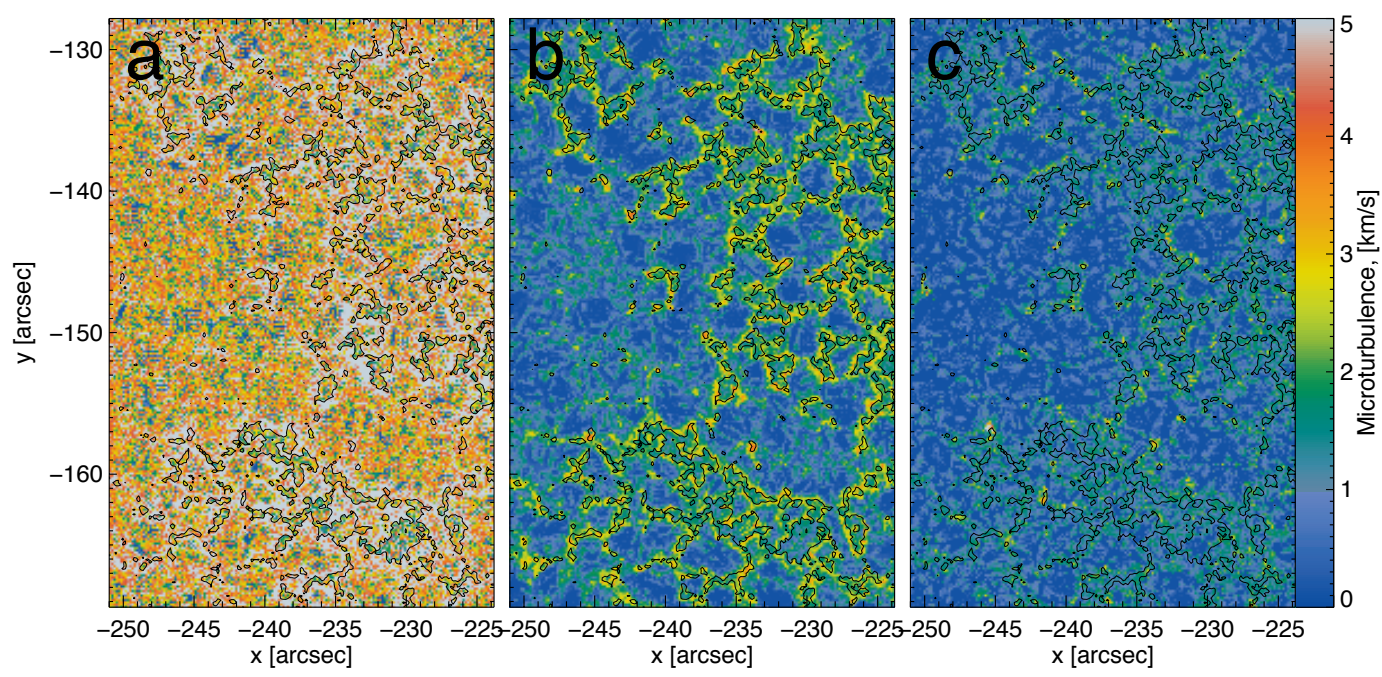

Figure 3.20: $a-c$ : The microturbulent velocities retrieved by the inversion at $\log (\tau)=$ $0,-0.9$ and -2.3 from left to right. All three images share the same colour scale. The black contour encompasses pixels where $B>900 G$ at $\log (\tau)=0$ in all images.

Therefore, we carried out the inversions with a height dependent microturbulence, while forcing the macroturbulence to zero. This approach turned out to give satisfactory fits to the line profiles. Restricting ourselves to microturbulence alone is also in line with the improved spatial resolution and stable observing conditions of the Hinode spacecraft. Any remaining non-thermal broadening in the spectral line profiles is assumed to be caused by unresolved velocities within the resolution element. A mixture of up- and downflows with a large correlation length along the LOS is less likely at high resolution, than in the low resolution data analyzed in the earlier investigations.

Figure 3.20 displays the microturbulent velocities, $\xi_{\text {mic }}$, retrieved by the inversion at the three $\log (\tau)$ heights. In all three panels areas with an increased $\xi_{\text {mic }}$ are found to coincide with areas of strong magnetic fields. In particular, Fig. $3.20 \mathrm{p}$ reveals that the greatest microturbulence can be found not only in regions of strong magnetic field ( $B>900 G$ at $\log (\tau)=0$ ), but that they are preferentially situated at the edges of a magnetic feature, forming a narrow halo around MFCs. A closer inspection of Fig. 3.20c reveals that the same is true at $\log (\tau)=-2.3$, although the halo is less well marked at this height. At $\log (\tau)=0$ the picture is only partly similar. A large number of plage pixels have microturbulent velocities of $5 \mathrm{~km} / \mathrm{s}$ irrespective of their location within the plage feature. Nonetheless, the presence of a now more extended halo of large microturbulence around the MFCs is unmistakeable.

In this inversion the upper limit for the microturbulence was set at $5 \mathrm{~km} / \mathrm{s}$. If a higher upper limit was set then microturbulent velocities of up to $9 \mathrm{~km} / \mathrm{s}$ were retrieved by the inversion at $\log (\tau)=0$. They were preferentially located near the edges of MFCs but hardly within them.

Histograms displaying $\xi_{\text {mic }}$, found in core pixels, are shown on the left in Fig. 3.21. These distributions can be compared to similar distributions depicting $\xi_{m i c}$ obtained from quiet Sun pixels, plotted on the right in Fig. 3.21. The differences in $\xi_{\text {mic }}$ between the quiet Sun and MFCs, suggested in Fig. 3.20, are confirmed by the distributions in Fig. 3.21. 

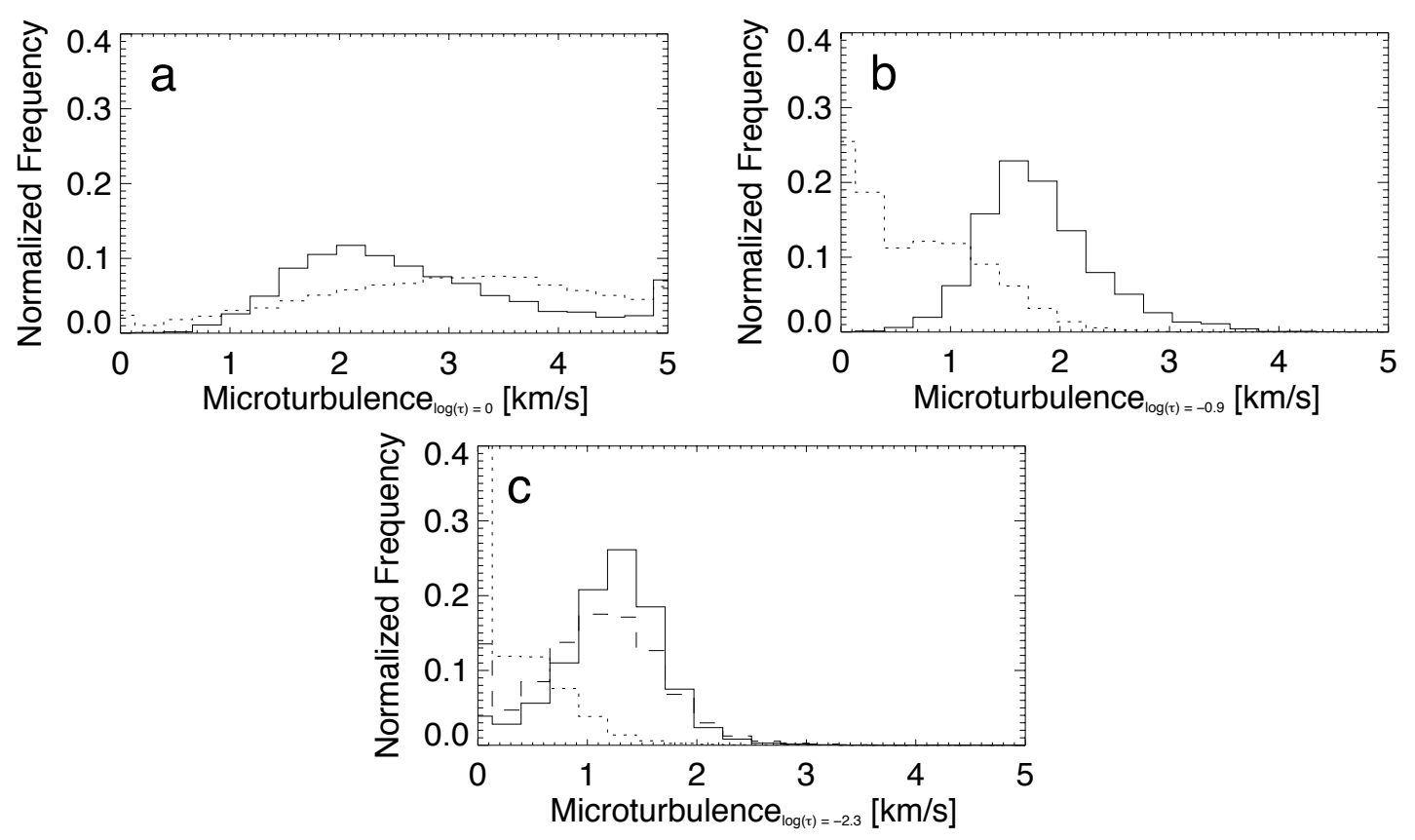

Figure 3.21: Histograms of microturbulent velocities in core pixels, solid and the quiet Sun, dotted, at $\log (\tau)=0, a$, at $\log (\tau)=-0.9, b$, and at $\log (\tau)=-2.3, c$. The dashed histogram shows the microturbulent velocities of canopy pixels at $\log (\tau)=-2.3$.

At $\log (\tau)=0 \xi_{\text {mic }}$ in the quiet Sun is on average slightly larger, with a mean velocity of $3.13 \mathrm{~km} / \mathrm{s}$, than in MFCs, which have an average velocity of $2.84 \mathrm{~km} / \mathrm{s}$. In higher layers the micro turbulence in MFCs is significantly larger than in the quiet Sun. In MFCs the average velocities are $1.94 \mathrm{~km} / \mathrm{s}$ and $1.33 \mathrm{~km} / \mathrm{s}$ at $\log (\tau)=-0.9$ and $\log (\tau)=-2.3$, whilst in the quiet Sun the average velocities are $0.83 \mathrm{~km} / \mathrm{s}$ and $0.38 \mathrm{~km} / \mathrm{s}$ in the two layers, respectively. Note that the canopy pixels require a similar microturbulence as the core pixels at the same optical depth. Fig. 3.21 also reveals that there are many pixels in the quiet Sun which require no microturbulent broadening at all, particularly in the upper two layers.

The microturbulence retrieved by the inversion does not necessarily imply the existence of turbulence or unresolved convective processes taking place within the magnetic features. It may point to unresolved waves in the MFC (e.g. surface waves could account for the higher $\xi_{\text {mic }}$ near the boundaries of the MFCs), or it may be due to unresolved horizontal velocity gradients strongest at the boundaries. Another possibility may be, at least to a part, a signal of jets produced by magnetic reconnection between the opposite polarity fields found in this study. Although it cannot be completely ruled out that inexactitudes in the damping constants of Fe I 6301.5 $\AA$ and 6302.5 (Anstee and O'Mara 1995, Barklem and O'Mara 1997; Barklem et al. 1998) may contribute to the deduced $\xi_{\text {mic }}$. However, such inexactitudes are unable to explain the excess in the $\xi_{\text {mic }}$ in the magnetic features, since the spectral lines are significantly weakened there, so that dampening becomes less important. However, the microturbulent velocities presented here do agree with the results obtained by both Solanki (1986) and Zayer et al. (1989) of excess turbulence in magnetic features. 


\subsection{Discussion and Conclusion}

In the preceding sections we sought to ascertain some of the characteristic properties of the magnetic field in solar plage. The observed Stokes $I, Q, U$ and $V$ profiles from the plage region observed by the SOT/SP aboard Hinode were analyzed using the SPINOR inversion code (Frutiger et al. 2000), which was extended by van Noort (2012) to perform a spatially coupled 2D inversion.

The inversion was able to retrieve and reproduce many of the characteristic properties previously observed in plages. The typical magnetic field strength of magnetic flux concentrations (MFCs) in plage was found to be in the $k G$ range and took a typical value of $1490 G$ at $\log (\tau)=-0.9$. Similar magnetic field values were found previously by Wiehr (1978); Zayer et al. (1990); Keller et al. (1990); Rüedi et al. (1992); Rabin (1992); Lin (1995); Martínez Pillet et al. (1997). These values are, however, somewhat larger than those found using the Fe I 5250.2/5247.1 A line pair (e.g. Stenflo and Harvey 1985). The magnetic field is found to drop more slowly with height than a thin-tube (zeroth order) model. This may have to do with the fact that some magnetic features merge with neighbouring MFCs already in the middle photosphere. Above the merging height $B$ drops more slowly or not at all (Pneuman et al.1986; Steiner et al. 1986). The fact that a zeroth order flux tube is too simple to describe a MFC (Yelles Chaouche et al. 2009) and expands more rapidly than more realistic models may also contribute.

The LOS velocities obtained by the inversion show that the bulk of the magnetized gas in MFCs is essentially at rest and shows only weak downflows on the order of $200 \mathrm{~m} / \mathrm{s}$, which agrees with the results of Solanki (1986); Martínez Pillet et al. (1997). Almost all MFCs are surrounded by a ring of downflows, which can be readily observed at the $\log (\tau)$ nodes 0 and -0.9 in Fig. 3.6. These downflows can take values of up to $8 \mathrm{~km} / \mathrm{s}$ at $\log (\tau)=0$, but typical values are between $1-3 \mathrm{~km} / \mathrm{s}$. Some of these downflows appear to overlap with the magnetic field, which could be the signature of entrainment, or may be a result of insufficient spatial resolution to cleanly resolve the magnetic boundary of MFCs. Similar downflows at the edges of magnetic features were found by Rouppe van der Voort et al. (2005) and Langangen et al. (2007). They are also compatible with the downflows inferred from the modeling of Stokes $V$ asymmetries (Grossmann-Doerth et al. 1988; Solanki 1989; Buente et al. 1993). These downflows are strongest at $\log (\tau)=0$ and gradually weaken higher up in the atmosphere and have completely disappeared at $\log (\tau)=-2.3$ suggesting that they are associated with the granular convection pattern. However, very strong downflows are more common at the boundaries of MFCs than in intergranular lanes far from plage.

Located in these downflow regions surrounding the MFCs we found small magnetic patches of the opposite polarity from the main MFC which they adjoin to. These small opposite polarity patches are only visible in the lower two $\log (\tau)$ nodes, as shown in Fig. 3.6, supporting the notion that they are intimately connected to the downflows in which they are immersed. MHD simulations carried out by Steiner et al. (1998) and Vögler et al. (2005) predict such patches in the vicinity of strong magnetic field concentrations. However, the reversal of the Stokes $V$ amplitude in the wings of the 630 $\mathrm{nm}$ line pair are only seen in the deconvolved profiles produced by the inversion. An additional complication is the masking of these magnetic fields by the canopy of the main plage feature. The canopy has a typical field strength of $300 G$ or more at locations overlying such weak opposite polarity 
fields. The canopy thus produces a strong signal in Stokes $Q, U$ and $V$, whereas the field strength of the opposite polarity patch at $\log (\tau)=0$ is barely stronger than $100 G$. This also prevents the selection of these patches via a typical amplitude threshold in the Stokes profile, so that we cannot completely rule out that even the magnetic fields $>100 G$ are an artifact of the inversion. The field strength of a small opposite polarity patch drops rapidly with height, producing an essentially field free region between it an the magnetic canopy above. Further observations performed with a higher spatial resolution are necessary to ascertain the existence of these small opposite polarity patches. Such observations would be particularly useful in the $1.56 \mu \mathrm{m}$ lines, due to their large Zeeman sensitivity and low formation height. The $1.56 \mu \mathrm{m}$ data analyzed by Zayer et al.(1989), although of low spatial resolution, provide some support for our results.

We have introduced a novel method for the resolution of the $180^{\circ}$ azimuth ambiguity. It makes use of the basic magnetic structure of MFCs and assumes that the divergence of the magnetic field is close to zero. The MFCs have typical inclinations relative to local solar coordinates between $10^{\circ}$ to $15^{\circ}$ in all three $\log (\tau)$ nodes of the inversion, which agree well with earlier inclination results found by Topka et al. (1992); Bernasconi et al. (1995) and Martínez Pillet et al. (1997). The distribution of the azimuths shows a preference for the eastern direction, which can be attributed to a LOS effect. MFCs located closest to the disc centre in the field of view showed the most homogeneous azimuth distribution, which supports the conclusion of Martínez Pillet et al. (1997) that in general MFCs have no preferred orientation. MFCs close to the sunspot did, however, display azimuth distributions that were either predominantly directed towards or away from the spot depending on their polarity. MFCs closest to the sunspot were found to have more inclined magnetic fields and azimuthal orientations that either pointed towards or away from the spot depending on the polarity. The canopies of these MFCs were also irregular and elongated. This demonstrates that the sunspot has a direct impact on the properties of MFCs even in the photosphere. The inversion was able to retrieve the locations of the magnetic canopies associated with the magnetic elements. A canopy could be identified for almost all MFCs and larger magnetic features as is demonstrated in Fig. 3.3. The canopy fields were found to harbour weaker more horizontal magnetic fields with inclinations as high as $\gamma=80^{\circ}$ and at least as low as the $300 G$ threshold at $\log (\tau)=-2.3$. The typical LOS velocities in the canopies are identical to the los velocities found at the centre of a magnetic feature. Many magnetic canopies were found to lie above essentially magnetic field free and quiet Sun regions. The canopies found here agree with the model proposed by Grossmann-Doerth et al. (1988) for the production of the Stokes $V$ area asymmetry and the observational results presented by Rezaei et al. (2007); Narayan and Scharmer (2010) and Martínez González et al. (2012). All magnetic features were observed to expand with height and many isolated MFCs merged to form expansive regions of magnetic field at $\log (\tau)=-2.3$. We therefore expect that at least some of the magnetic features display a similarity to the wine-glass model described by Buente et al. (1993).

The expansion of seven isolated MFCs was compared to the ideal expansion of a $0^{\text {th }}$ order thin flux tube model. The expansion of the selected magnetic features and the model agreed well, supporting previous results reported by Pietarila et al. (2010). At the highest inverted layers the field did expand somewhat less rapidly than in the model, however, possibly due to magnetic curvature forces, or due to the interactions with other magnetic features. We also compared the magnetic flux at different heights. Since we independently 
determine the gradient in $B$ (in each pixel) and the expansion of the MFCs, which involves comparing neighbouring pixels, ours is an independent test of the conservation of magnetic flux with height. We find that the flux differs by only $20 \%$ between $\log (\tau)=0$ and -2.3 . This difference may be due to some cancellation of Stokes $V$ signal at $\log (\tau)=0$ and -0.9 caused by the presence in these layers of fields next to the MFC with a polarity opposite to that of the MFC.

Finally, the inversions worked well with a depth-dependent microturbulent velocity, allowing the macroturbulence to be set to zero. Particularly high values of the microturbulence were found at the edges of the magnetic features, reaching values in excess of $5 \mathrm{~km} / \mathrm{s}$ at $\log (\tau)=0$. The typical microturbulent velocities in MFCs obtained by the inversion agree with the results presented by Solanki (1986) and Zayer et al. (1989). This suggests the presence of strong unresolved velocities within the magnetic features. Also, although their amplitude decreases rapidly with height, $\xi_{\text {mic }}$ is still at least a factor of 2 larger in the MFC than outside in the upper two $\log (\tau)$ layers. These large microturbulence values may be telling us that the strong downflows at the edges of the MFCs are associated with vigorous turbulent motions, or it may be a signature of surface waves travelling along the boundary of the MFC. Finally, the microturbulence returned by the inversion code may be signalling a strong horizontal velocity gradients e.g. across the boundary of MFCs such as with a strong downflow outside and a weak one inside.

This investigation has demonstrated the value of the inversion technique developed by van Noort (2012) for probing MFC forming solar plage. It has allowed us to test and confirm independently a number of their properties, but has also uncovered new aspects, such as the presence of weak opposite polarity fields around MFCs. 


\section{Analysis of inversion based azimuth rotation in plage across the solar disc}

\subsection{Introduction}

This chapter decribes a direct follow-up investigation of the study presented in chapter 3 . In particular the distributions of the azimuthal orientation of the magnetic fields, presented in section 3.5.4, warranted and required further investigation. The distributions indicated that the azimuthal angles of the magnetic field were not constant but appeared to vary with height, implying a rotation or twist of the magnetic field. In chapter 3 it was concluded that this rotation is most likely an artifact of the inversion and not solar in origin. This chapter focusses on why it is important to investigate the twist in solar magnetic fields and on the tests performed to determine if the observed twists are indeed an artifact.

Hale (1927), studying $H \alpha$ images, postulated a law now known as Hale's helicity law, which states: Independent of the solar cycle ARs in the northern solar hemisphere show an anti-clockwise twist in the magnetic field whereas ARs in the southern hemisphere show a clockwise twist (Richardson, R. S. 1941). In classical dynamo theory it is the Coriolis force that imparts a helicity on rising magnetic flux tubes and also requires the reversal of this helicity at the equator (Parker, E. N. 1955). The helicity of ARs and sunspots can be estimated from the $B_{x}, B_{y}$ and $B_{z}$ components of the magnetic field derived from a potential field extrapolation or vector magnetograms (Seehafer 1990). Pevtsov et al. (1995) tested this law by studying magnetograms of 60 ARs recorded from 1988 - 1994 and showed that $\approx 70 \%$ of ARs follow Hale's law. The helicity was found to reach its maximum at latitudes $10^{\circ}-15^{\circ}$, while the helicity tends towards zero at the solar equator and poles. Nonetheless significant deviations from this rule are common. Abramenko et al. (1996) found studying ARs, using vector magnetograms, that $90 \%$ of them possess a significant helicity and in their case $\approx 80 \%$ of ARs followed Hale's rule. Choudhuri et al. (2004) used the Babcock-Leighton dynamo model to suggest that the deviations from Hale's law may have a cycle dependence. Tiwari et al. (2009) on the other hand found, using Hinode SOT/SP images of ARs, that most ARs at the end of cycle 23 did not appear to follow Hale's law, and Pevtsov et al. (2008) reported that large data set is needed to reliably determine the helicity in a hemisphere at a given time.

Whilst the previous studies either investigated whole ARs or examined large sunspots, other authors have studied the dynamics of individual thin flux tubes, covering a fraction of an arcsecond in extent, to infer a potential twist in their magnetic field. Since the lateral extent of these features is usually of the same order as the telescope resolution, the helicity of these features is difficult to determine from polarimetric measurements 
alone. Therefore, the helicity is inferred by proxy whereby the dynamics of the tube are analyzed using a local correlation method or direct tracing (Brandt et al. 1988; Bonet et al. 2008, 2010; Balmaceda et al. 2010). Many flux tubes are caught in vortex flows, which occur at the boundaries of granules and supergranules where the cool plasma sinks into the solar interior (Attie et al. 2009). Any flux tube immersed in such a vortex would have its magnetic field wound up and twisted (Wedemeyer-Böhm et al.|2012). However, unlike ARs, the vortices at these granular boundaries should have no hemispheric dependence, since they are essentially a local phenomenon.

Recent simulations of thin flux tubes have also shown vortices to exist at the interface between the tube and the surrounding magnetic field-free plasma (Moll et al. 2011). Moll et al. (2012) further showed that these vortices are capable of heating the upper photosphere and chromosphere. However, their small size means that they cannot be spatially resolved with current solar telescopes.

Based on the works cited in this section it is clear that detection of twisted magnetic fields on the solar surface would be neither controversial nor unheard of. However, the direct measurement of helicity derived from Stokes $Q, U$ and $V$ would be new and would offer a new way of measuring the magnetic helicity in features smaller than sunspots.

\subsection{Data and Inversions}

The data sets used in this investigation were recorded by SOT/SP (Tsuneta et al. 2008b; Suematsu et al. 2008; Ichimoto et al. 2008; Shimizu et al. 2008) aboard Hinode (Kosugi et al. 2007). All the ARs were scanned in normal mode with an exposure time of $4.8 \mathrm{~s}$ per slit position and an angular resolution of 0."3. All four Stokes parameters, $I, Q, U$ and $V$, were recorded at each slit position. Active regions scanned in 2006 and 2007 were recorded using a dual beam set up, whereby both polarisation states of the Stokes $Q, U$ and $V$ parameters were recorded simultaneously. From 2008 onwards SOT/SP operated in a single beam mode where the polarisation states of the Stokes $Q, U$ and $V$ parameters were obtained one after the other. This change in the operational setup was necessitated by a loss of telemetry of the satellite from 2008 onwards. Due to the stable observing conditions experienced by SOT/SP, the depreciation of the polarimetric data inflicted by this change is minimal. The variation in the Stokes $I \mathrm{rms}$ contrast with time and its effect on the polarisation signals, described in Chapter 2, was not compensated for. Unlike the weak polarisation signals studied in Chapter 2, which are very susceptible to small variations within the satellite, the polarimetric signals studied in this investigation were far larger. All the polarimetric signals considered in this investigation had a Stokes $Q$, $U$ or $V$ amplitude of at least $5 \sigma$ or more, making the effect of the temperature variation upon the polarisation signals within the satellite negligible. The data were reduced using the standard $s p \_$prep routine (Lites et al. 2008; Lites and Ichimoto 2013) from the solar software package, followed by a bitshift correction of the four Stokes parameters.

All the SOT/SP images were inverted by the SPINOR code (Frutiger et al.2000), which was extended by van Noort (2012) to perform a spatially coupled inversion of the Stokes spectra. The inversion procedure used for each AR was identical to the one described in Chapter 3. Apart from AR 10953, which had $\log (\tau)$ nodes set at $0,-0.9$ and -2.3 , all the other ARs were inverted with $\log (\tau)$ nodes $0,-0.9$ and -2.5 . The quality of the fits to the 
observed spectra brought about by this change in the placement of the upper $\log (\tau)$ node is minimal since the contribution function of the $6300 \AA$ line pair changes only marginally between $\log (\tau)=-2.3$ and -2.5 .

The data employed in this investigation is listed in Table 4.1. The images used cover active regions from cycles 23 and 24 in both hemispheres on the solar disc. The images belonging to cycle 23, recorded in 2007 and 2008, were located predominantly in the southern hemisphere due to a lack of activity in the northern hemisphere during that time. Sometimes an active region was recored several times on different days as it moved across the solar disc. These multiple recordings of the same active region offered the opportunity to analyze the potential change in twist in an active region with respect to time. One image used in this investigation contained only network magnetic fields and quiet Sun and, therefore, did not belong to an active region.

Table 4.1: SOT/SP twist analysis images

\begin{tabular}{cccccc}
\hline \hline Image & Date & Hemisphere & $\mu^{1}$ & Active Region & Twist $^{2}$ \\
\hline 1 & 2006 Dec 03 & $\mathrm{S}$ & 0.89 & 10930 & $50^{\circ}$ \\
2 & 2007 Jan 09 & $\mathrm{S}$ & 0.69 & 10933 & $10^{\circ}$ \\
3 & 2007 Jan 31 & $\mathrm{S}$ & 0.96 & 10940 & $40^{\circ}$ \\
4 & 2007 Mar 01 & $\mathrm{S}$ & 0.98 & 10944 & $40^{\circ}$ \\
5 & 2007 Mar 10 & $\mathrm{S}$ & 0.99 & - & $30^{\circ}$ \\
6 & 2007 Apr 30 & $\mathrm{S}$ & 0.97 & 10953 & $50^{\circ}$ \\
7 & 2007 May 18 & $\mathrm{N}$ & 0.97 & 10956 & $15^{\circ}$ \\
8 & 2007 May 20 & $\mathrm{N}$ & 0.97 & 10956 & $15^{\circ}$ \\
9 & 2007 Dec 13 & $\mathrm{S}$ & 0.94 & 10978 & $30^{\circ}$ \\
10 & 2009 Dec 28 & $\mathrm{S}$ & 0.86 & 11039 & $25^{\circ}$ \\
11 & 2010 Jan 26 & $\mathrm{S}$ & 0.94 & 11041 & $50^{\circ}$ \\
12 & 2010 Sep 16 & $\mathrm{S}$ & 0.89 & 11106 & $60^{\circ}$ \\
13 & 2010 Oct 26 & $\mathrm{N}$ & 0.95 & 11117 & $45^{\circ}$ \\
14 & 2011 Apr 19 & $\mathrm{N}$ & 0.93 & 11193 & $35^{\circ}$ \\
15 & 2011 Nov 28 & $\mathrm{N}$ & 0.92 & 11358 & $30^{\circ}$ \\
16 & 2011 Nov 30 & $\mathrm{N}$ & 0.96 & 11361 & $20^{\circ}$ \\
17 & 2011 Dec 06 & $\mathrm{S}$ & 0.88 & 11363 & $40^{\circ}$ \\
18 & 2012 Feb 18 & $\mathrm{N}$ & 0.81 & 11419 & $40^{\circ}$ \\
19 & 2012 Jul 31 & $\mathrm{S}$ & 0.81 & 11536 & $20^{\circ}$ \\
20 & 2012 Sep 01 & $\mathrm{N}$ & 0.99 & 11560 & $10^{\circ}$ \\
& & & & & \\
\hline
\end{tabular}

\footnotetext{
${ }^{1} \mu=\cos (|\theta|)$, where $\theta$ is the heliocentric angle

2 Average twist according to Eq. 4.1, all twists are clockwise twists
} 


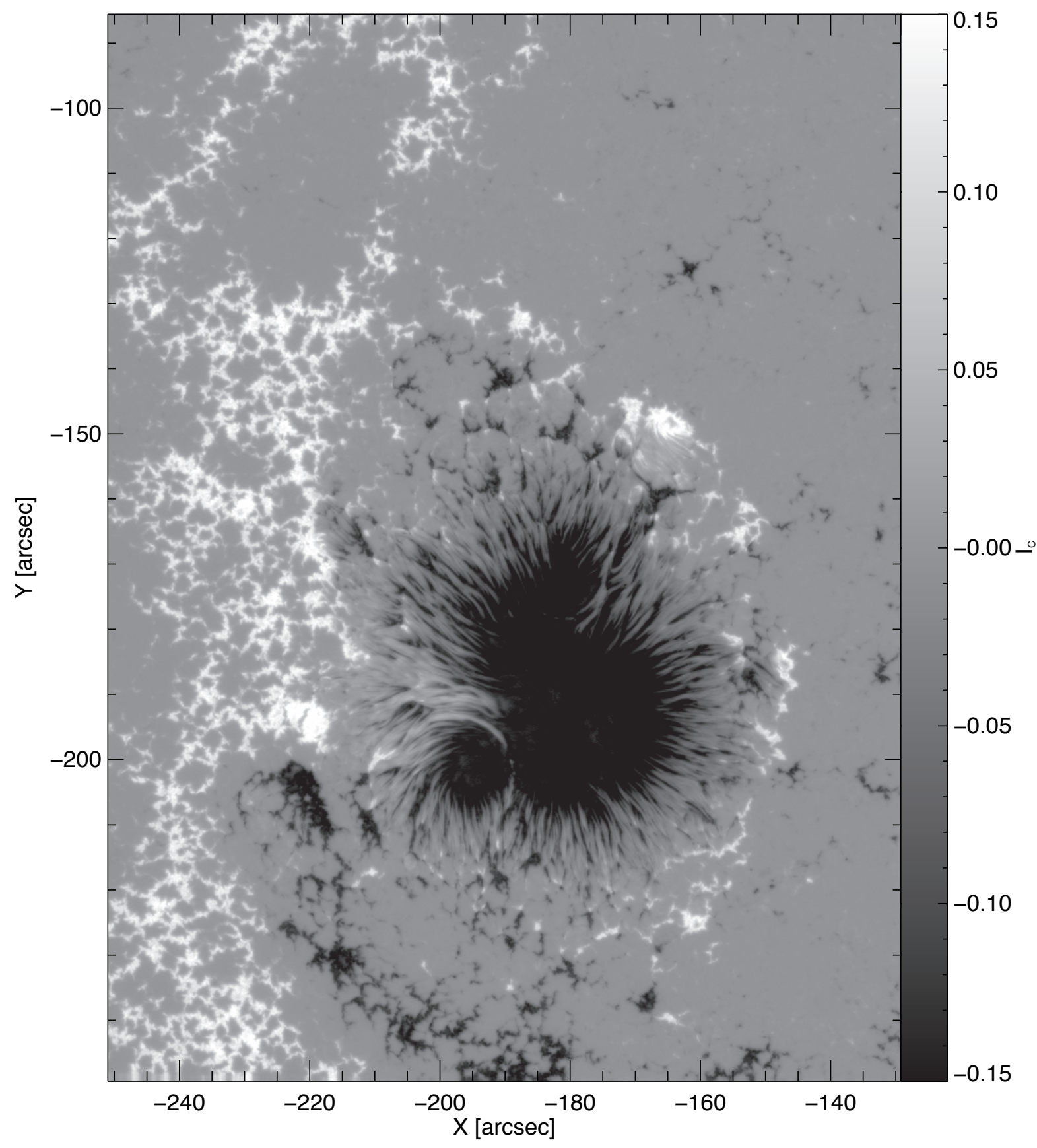

Figure 4.1: Normalised Stokes $V$ image of AR 10953 recorded by Hinode SOT/SP on the $30^{\text {th }}$ April 2007 at UT 18:35:18.

\subsection{Twist in AR 10953}

This section examines both the inclinations and azimuths of the magnetic fields found in plage of AR 10953 presented in section 3.5.4. A Stokes $V$ image of the AR, recorded by Hinode SOT/SP, can be seen in Fig. 4.1. The AR presents a complex arrangement of both polarities at the time of the observation. Fig. 4.2 shows the azimuths and inclinations of some of the magnetic flux concentrations (MFCs) belonging to this AR. The $180^{\circ}$ azimuth 

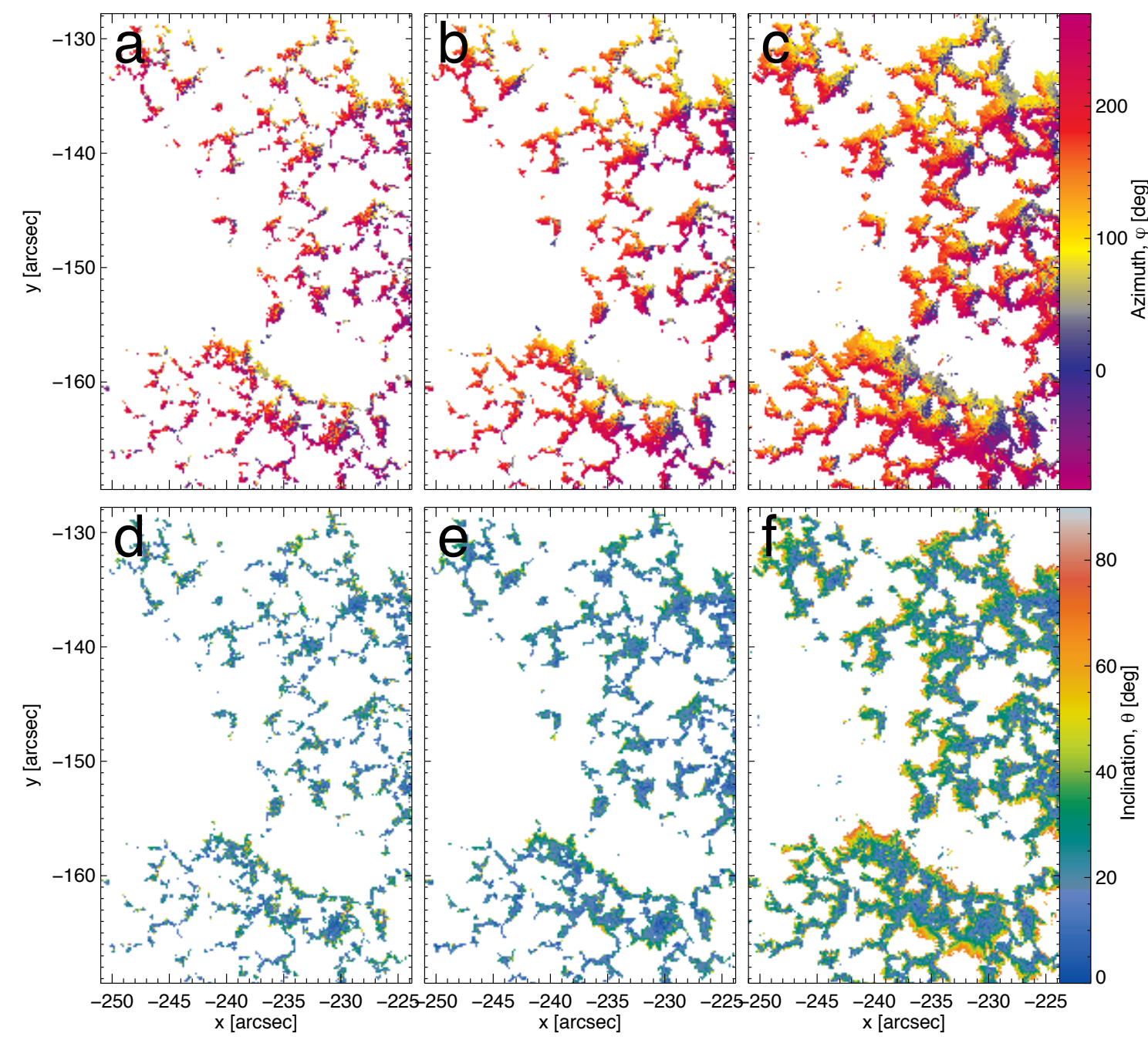

Figure 4.2: Top row: The resolved local solar coordinate corrected azimuths, $\varphi$, at $\log (\tau)=$ $0,-0.9$ and -2.3 from left to right. North is up and corresponds to an angle of $90^{\circ}$. West is to the right and corresponds to an angle of $0^{\circ}$. Bottom row: The local solar coordinate corrected inclinations, $\theta$, of the magnetic field after the azimuth ambiguity resolution at $\log (\tau)=0,-0.9$ and -2.3 from left to right. The inclinations and azimuths of pixels with $B<300 G$ at $\log (\tau)=-2.3$ and $B<700 G$ at $\log (\tau)=0 \&-0.9$ are shown in white.

ambiguity was removed and all the inclinations are relative to the normal on the local solar surface. The definitions of core and canopy employed in chapter 3 are carried over into this investigation. In summary a core pixel must have a magnetic field strength, $B$, of at least $900 G$ at $\log (\tau)=0$ as well as a negative magnetic field gradient with height. A canopy pixel has a $B$ of $300 G$ at $\log (\tau)=-2.3$ and a positive $\Delta B$ with height. At all times, the pixels used in the following analysis have a Stokes $Q, U$ or $V$ amplitude of at least $5 \sigma$, where $\sigma=1 \times 10^{-3} I_{c}$. By selecting both the core and canopy pixels, histograms of their azimuth distribution at each $\log (\tau)$ height can be produced. The plot on the left in Fig. 4.3 shows the azimuth distributions for core pixels and canopy at $\log (\tau)=-2.3$. All the pixels had the same polarity and excluded pores. The distributions of the core pixels show not only a preferred orientation, which is a LOS effect, but also the peak of each 

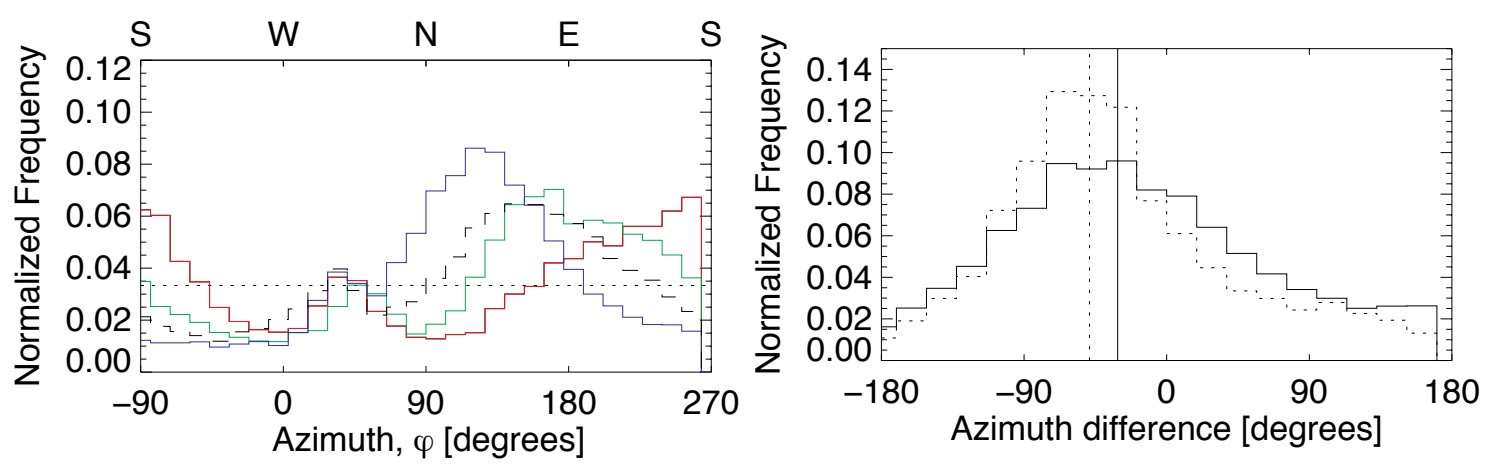

Figure 4.3: Left: Histograms of $\varphi$ found in positive polarity plage. The three coloured histograms were obtained using core pixels, where red refers to $\log (\tau)=0$, green shows to $\log (\tau)=-0.9$ and blue refers to $\log (\tau)=-2.3$. The dashed histogram shows $\varphi$ of canopy pixels at $\log (\tau)=-2.3$. The dotted line represents a homogeneous distribution. Right: Azimuth difference calculated using Eq. 4.1.

distribution is shifted with respect to each other. The relative shift between these peaks suggests a rotation in the azimuthal orientation of the magnetic field with height or a twist. This was investigated further by calculating the azimuth difference, $d_{\varphi}$, separately for each pixel where,

$$
d_{\varphi}=\varphi_{\log (\tau)=-2.3}-\varphi_{\log (\tau)=0} .
$$

Since the peak of $\varphi$ at $\log (\tau)=-0.9$ lies between the other two histograms the definition of $d_{\varphi}$ appears reasonable. The $d_{\varphi}$ values from the core pixels were compiled in a histogram and are shown by the solid distribution in the right plot in Fig. 4.3. The peak of the distribution is not centred around zero, which indicates that the azimuthal direction of the magnetic field vector in plages changes with height. A simple Gaussian fit to the solid distribution gives $\left\langle d_{\varphi}\right\rangle=-31^{\circ} \pm 4^{\circ}$, shown by the solid vertical line in the right plot in Fig. 4.3. and standard deviation $\sigma=86^{\circ} \pm 4^{\circ}$. The Gaussian fit confirms the twist indicated in the left plot in Fig. 4.3. However, it is important to remember that the core pixels are composed of predominantly vertical fields, as Fig. 4.2 demonstrates. Therefore, the Stokes $Q$ and $U$ profiles tend to be weak, which in turn makes $\varphi$ unconstrained (Unno 1956). If the twist is to be given any credibility it needs to be present in pixels with strong Stokes $Q$ or $U$ profiles as well.

Core pixels with a Stokes $Q$ or $U$ amplitude of at least $5 \sigma$ were selected and their $d_{\varphi}$ was computed. The resulting histogram is shown by the dotted distribution in the right plot in Fig. 4.3. The distribution was once again fitted by a simple Gaussian and yields $\left\langle d_{\varphi}\right\rangle=-49^{\circ} \pm 4^{\circ}$ and $\sigma=57^{\circ} \pm 4^{\circ}$. This shows that when rigid constraints are applied to the Stokes $Q$ and $U$ profiles the twist does not vanish but rather becomes better defined. When even higher Stokes $Q$ or $U$ thresholds are set, progressively only values of $d_{\varphi}$ clustered around $-50^{\circ}$ remain.

The apparent twist was analyzed further by looking at plages of the opposite polarity found around $-220 Y$ and $-215 X$ in Fig. 4.1. The $\varphi$ of core pixels found in this region are shown on the left plot in Fig. 4.4. Once again, the peaks of the distributions are shifted with respect to each other, suggesting that the azimuthal direction of the magnetic field vector is rotating. Notably though, the rotation appears to be in the opposite sense to the one shown in the right plot in Fig. 4.3 . 

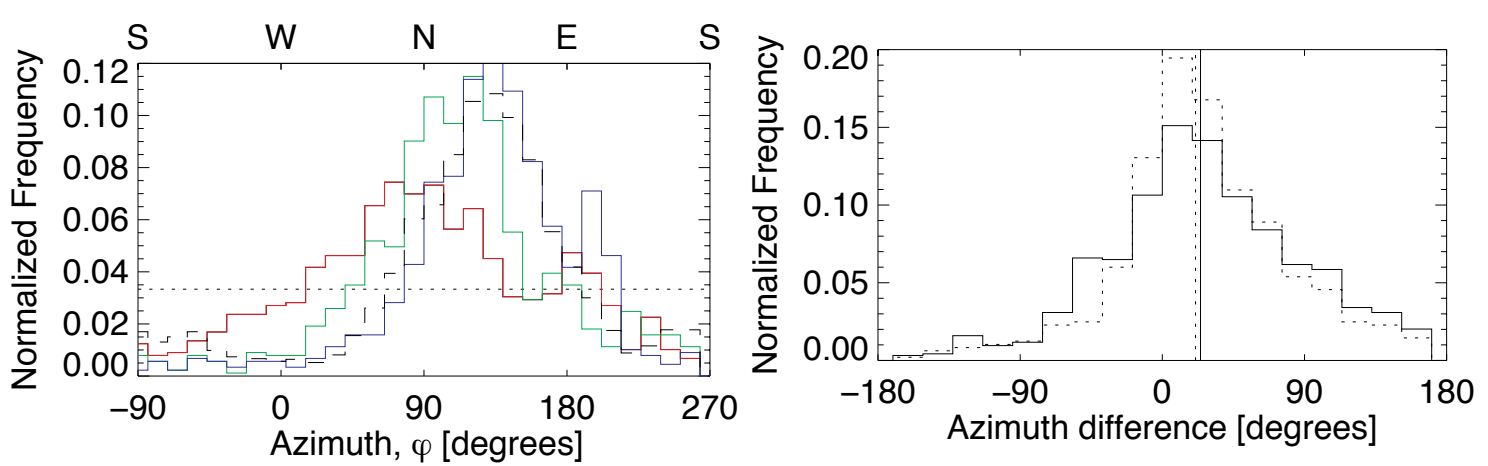

Figure 4.4: Left: Histograms of $\varphi$ found in negative polarity plage. The three coloured histograms were obtained using core pixels, where red refers to $\log (\tau)=0$, green shows to $\log (\tau)=-0.9$ and blue refers to $\log (\tau)=-2.3$. The dashed histogram shows $\varphi$ of canopy pixels at $\log (\tau)=-2.3$. The dotted line represents a homogeneous distribution. Right: Azimuth difference calculated using Eq. 4.1.

This opposite sense of rotation is illustrated further by computing the $d_{\varphi}$ of these core pixels. The solid histogram on the right in Fig. 4.4 shows the distribution of all the core pixels, whereas the dotted histogram corresponds again to those core pixels with a $5 \sigma$ Stokes $Q$ or $U$ amplitude. Both distributions were fitted by simple gaussians and the solid distribution has $\left\langle d_{\varphi}\right\rangle=23^{\circ} \pm 3^{\circ}$ and $\sigma=52^{\circ} \pm 3^{\circ}$, whereas the dotted histogram yields $\left\langle d_{\varphi}\right\rangle=21^{\circ} \pm 3^{\circ}$ and $\sigma=40^{\circ} \pm 3^{\circ}$. Importantly, setting a more stringent Stokes $Q$ or $U$ threshold does not significantly decrease the apparent twist.

Figs. $4.3 \& 4.4$ present the following coherent picture concerning the twist in the magnetic field. There appears to be an overall clockwise twist in all the plage analyzed here. The apparent counter-clockwise in Fig. 4.4 twist is to be expected for fields in a scenario where a (idealistic) cylinder of magnetic fields bearing a clockwise twist is bent in order to loop back on itself.

A common way to express the twist in a sunspot is the twist parameter $\alpha$ where $B \alpha=\nabla \times B$ (Seehafer 1990). The parameter $\alpha$ is positive (negative) when a clockwise (counterclockwise) twist is present in the magnetic field. Following Tiwari et al. (2009) we estimate the twist using a 'global' twist parameter, $\alpha_{g}$, where,

$$
\alpha_{g}=\frac{\Sigma(\nabla \times B)_{z} B_{z}}{\Sigma B_{z}^{2}} .
$$

For the core pixels with a Stokes $Q$ or $U$ amplitude $>5 \sigma$ we find $\alpha_{g}=6.5 \times 10^{-8} \mathrm{~m}^{-1}$ for plages with positive magnetic polarity and $\alpha_{g}=8.7 \times 10^{-8} \mathrm{~m}^{-1}$ for plages with negative polarity in the field of view. This confirms the clockwise twist inferred from Figs. 4.3 $\& 4.4$ and corresponds to a positive helicity. Furthermore, previous $\alpha_{g}$ measurements for sunspots Tiwari et al. (2009) finds typical values between $1 \times 10^{-7} \mathrm{~m}^{-1}$ and $1 \times 10^{-9} \mathrm{~m}^{-1}$. The $\alpha_{g}$ values calculated for the plages support Hale's helicity law (Hale 1927) and are in agreement with Pevtsov et al. (1995); Abramenko et al. (1996) who showed that the majority of active regions follow this law. However, care must be taken when evaluating the significance of the $\alpha_{g}$, since the $(\nabla \times B)_{z}$ term in Eq. 2 was calculated along a constant $\log (\tau)$ surface rather than a constant geometrical height as Eq. 2 requires. In order to minimize this source of error, the $\alpha_{g}$ values were calculated at $\log (\tau)=-2.3$. Also the 

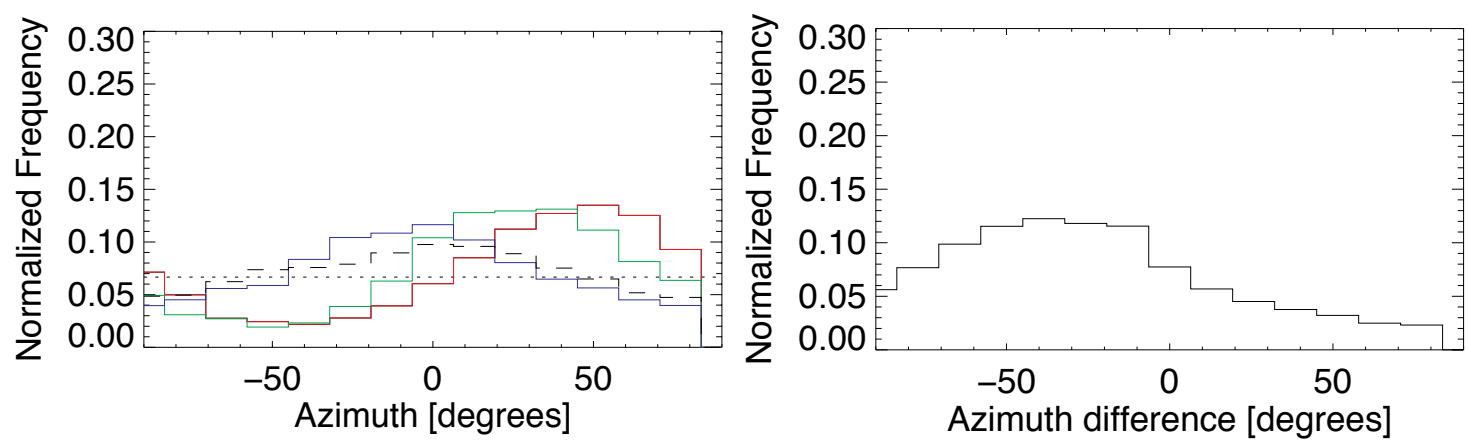

Figure 4.5: Left: Histograms of $\psi$ found in positive polarity plage. The three coloured histograms were obtained using core pixels, where red refers to $\log (\tau)=0$, green shows to $\log (\tau)=-0.9$ and blue refers to $\log (\tau)=-2.3$. The dashed histogram shows $\psi$ of canopy pixels at $\log (\tau)=-2.3$. The dotted line represents a homogeneous distribution. Right: Azimuth difference calculated using Eq. 4.1.

number of plage pixels with an ambiguity resolved magnetic field vector was largest at $\log (\tau)=-2.3$.

An immediate question to the results presented in this section is whether the twist is introduced by accident during the azimuth ambiguity removal routine. This concern was tested for and Fig. 4.5 shows the azimuths of the same core pixels as used in Fig. 4.3 but the azimuth ambiguity has not been removed. The figure demonstrates that the ambiguity removal routine does not introduce the twist, nor does it appear to alter the $\left\langle d_{\varphi}\right\rangle$ in the plages. Also, the apparent anti-clockwise twist in Fig. 4.4 was discernible in the azimuth ambiguous distributions (not shown here). Since the azimuth removal routine is based on an acute angle method which seeks to minimize the difference between neighbouring vectors, it would by default attempt to minimize any twist present in the data. Fig. 4.5 proves this initial assumption to be correct since the magnitude and direction twist is left unchanged by the azimuth removal routine. From this it was also concluded that it was not necessary to resolve the azimuth in order to be both able to discern the existence of a twist in plages and its direction (clockwise or anti-clockwise).

The results presented in this section indicate that there is a twist in the magnetic fields in plage. The fact that this twist is present across many different individual MFCs making up the plage further suggests that the twist is unlikely to be due to a local solar effect, such as granular vortices. The clockwise direction of twist agrees with Hale's law and the $\alpha_{g}$ values retrieved agree well with the values obtained by Tiwari et al. (2009), suggesting that the inversion is indeed capable of retrieving the twist in magnetic fields directly from the Stokes spectra.

\subsection{Twist in ARs across the solar disc}

The results obtained in Sect. 4.3 caused an investigation aimed to both determine whether a twist can also be found in other ARs inverted by SPINOR and if so, whether the twist has a hemispherical dependence in accordance with Hale's law. The data sets used for this section are listed in Tab. 4.1. In total 12 data sets found in the southern hemisphere and 8 


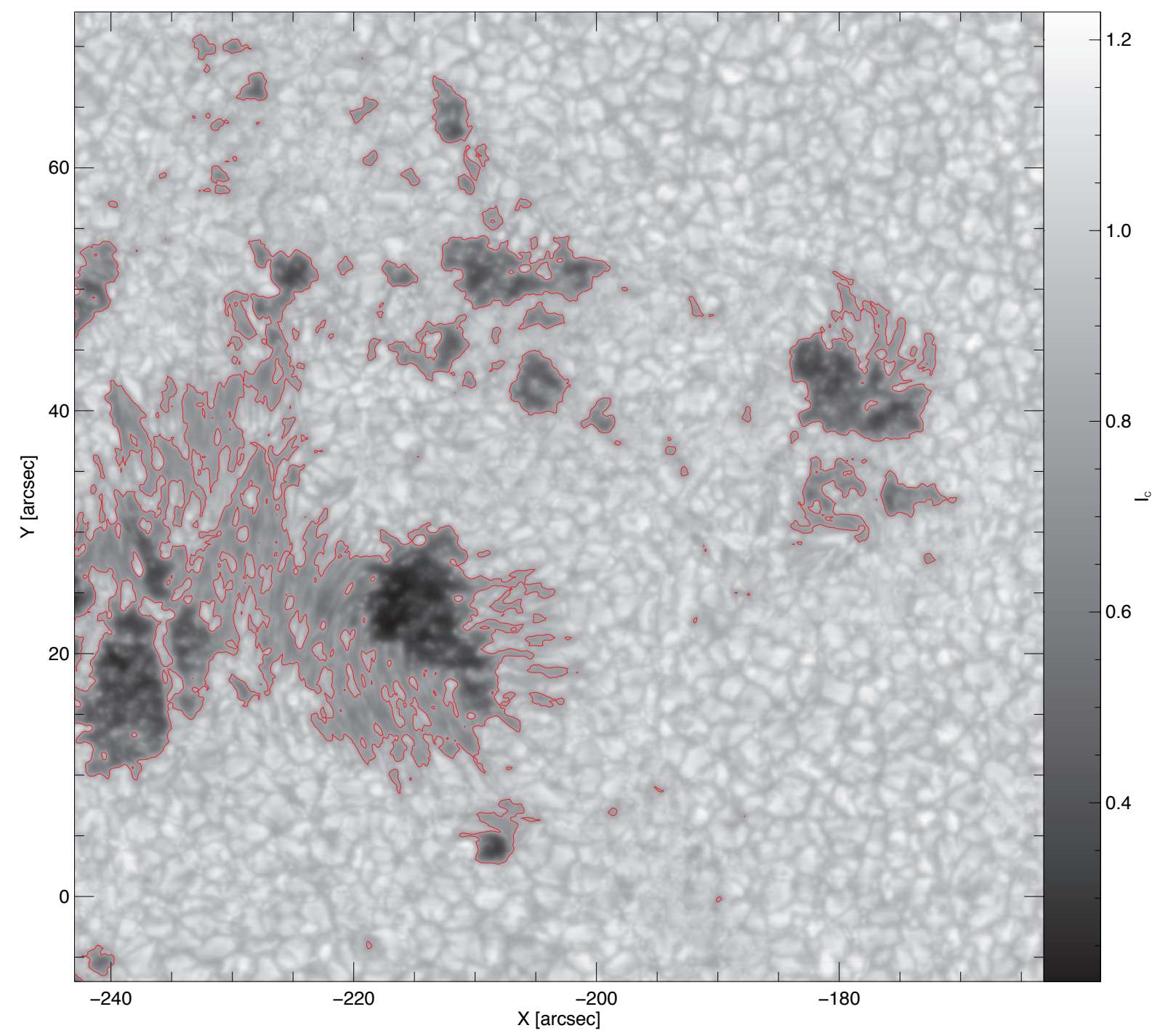

Figure 4.6: Continuum image of AR 10956 recorded by Hinode SOT/SP on the $18^{\text {th }}$ May 2007 at UT 12:00:07. The red contour encompasses areas where $I_{c}<0.8\left\langle I_{c}\right\rangle$.

data sets from the northern hemisphere were investigated, covering the end of cycle 23 and the beginning of cycle 24 .

A continuum image of AR 10956 used in this investigation is shown in Fig. 4.6. The AR emerged at the end of cycle 23 and was therefore located very close to the solar equator. In fact one of the pores of the AR is situated below the solar equator. Nonetheless, using Stokes $V$ it was possible to determine that this AR belonged to the northern hemisphere. Fig. 4.6 displays a very complex penumbral structure and some of the penumbral filaments appear to curl, indicating that there may be a global twist in the magnetic field. There were several other ARs listed in Tab. 4.1 that had a similar complex structure as the one shown in Fig. 4.6.

The magnetic fields in plage regions of every AR listed Tab. 4.1 were selected based on the same selection criteria used in Sect. 3 in order to make the results comparable. All the ARs showed twisted magnetic fields, which proved that the twisted magnetic fields 

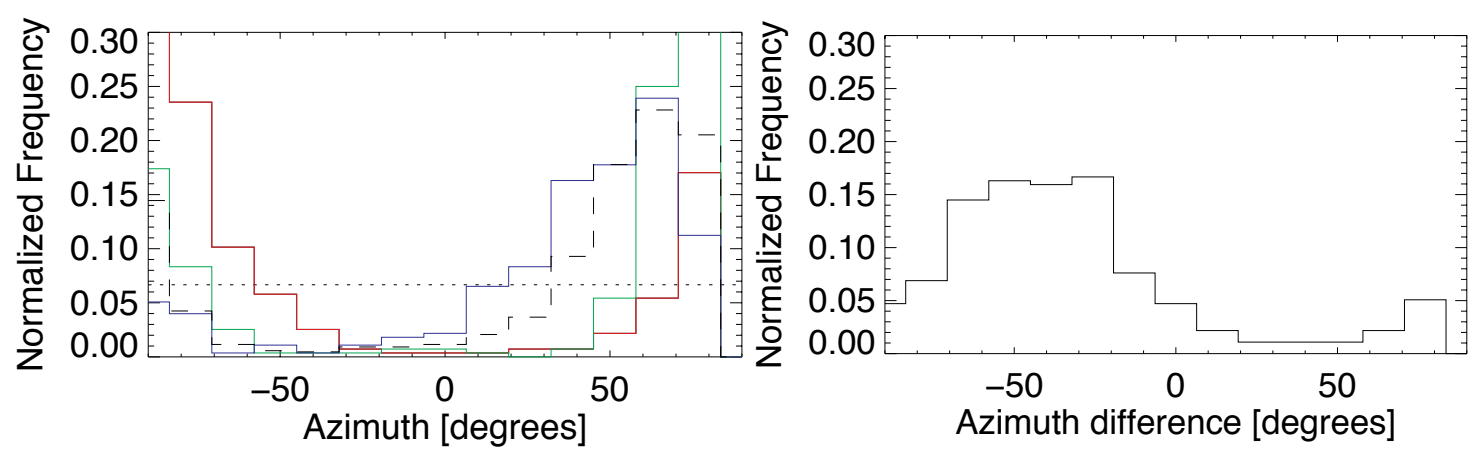

Figure 4.7: Left: Histograms of $\psi$ found in typical plage. The three coloured histograms were obtained using core pixels, where red refers to $\log (\tau)=0$, green shows to $\log (\tau)=$ -0.9 and blue refers to $\log (\tau)=-2.3$. The dashed histogram shows $\psi$ of canopy pixels at $\log (\tau)=-2.3$. The dotted line represents a homogeneous distribution. Right: Azimuth difference calculated using Eq. 4.1.

found by SPINOR in AR 10953 were not an isolated case. A typical example of twisted magnetic fields in an AR other than 10953 can be seen in Fig. 4.7. The peak in the right hand plot in Fig. 4.7 is comparable to the dotted distribution in the right plot in Fig. 4.5. A threshold of $5 \sigma$ in either the Stokes $Q$ or $U$ amplitude was imposed prior to the selection of the pixels. This demonstrates that, similar to the plage in AR 10953, the twist in plage of the ARs was not the result of poorly constrained azimuths due to weak Stokes $Q$ or $U$ signals. Not all ARs had the peak of their azimuth distribution around $50^{\circ}$, but were spread between $10^{\circ}$ to $60^{\circ}$, see Tab. 4.1. The largest azimuth difference peak values were generally found in those ARs belonging to cycle 23, such as Fig. 4.5.

While the majority of ARs studied here were observed close to the disc centre, AR 10933 was observed at $\mu=0.69$. As a result the MFCs belonging to plage were very inclined with respect to the line of sight. The twist in the unresolved azimuth of these inclined magnetic fields was very weak, peaking at $5^{\circ}$. The reduction of azimuthal twist with decreasing $\mu$ is expected, since for small $\mu$ values Stokes $Q$ and $U$ measure the inclination of the magnetic field rather than its azimuth in the instrument's reference frame.

However, even though all MFCs found in plage in the ARs listed in Tab 4.1 show twisted magnetic fields, they all possess a clockwise twist and never show an anticlockwise twist. This clearly speaks against Hale's law and has, as a consequence, given weight to the notion that an artifact of the inversion or a residual effect in the measured Stokes parameters might be responsible for the twist.

\subsection{Cross talk}

In this section we investigate whether a residual cross talk between the measured Stokes parameters could be responsible for inducing a twist in the inversion results. A cross talk between the Stokes parameters can occur easily, since many optical components within the satellite can alter the polarization of the incoming light. These effects are typically taken into account during the data reduction process by applying the telescope calibration model. The azimuth distributions in Figs. 4.3 \& 4.4 display an apparent clockwise and an anticlockwise twist, respectively. For Fig. 4.3 the Stokes $V$ polarity was positive whilst for 


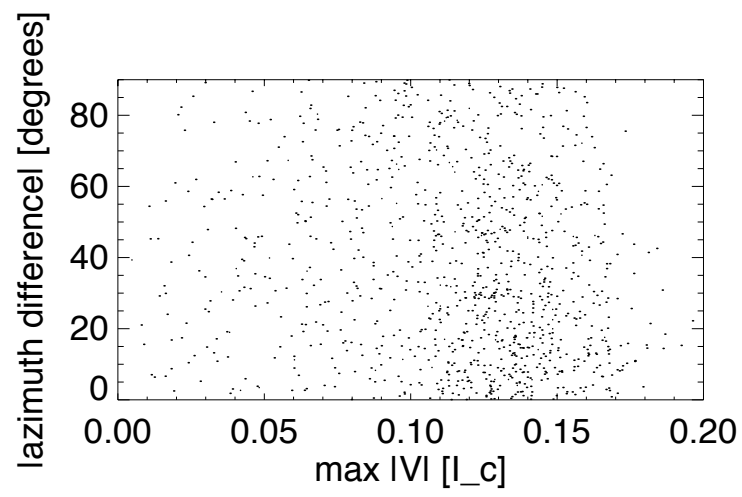

Figure 4.8: Stokes $V$ cross talk in core plage pixels from AR 10953 with a correlation coefficient of $r=0.1$.

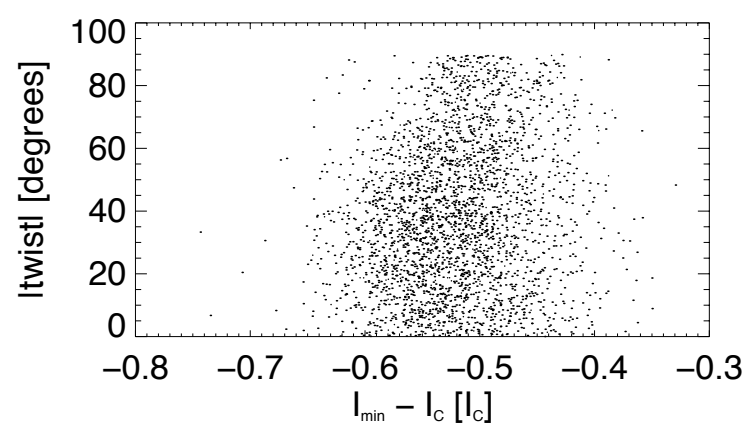

Figure 4.9: Stokes $I$ cross talk in core plage pixels from AR 10953 with a correlation coefficient of $r=0.2$.

Fig. 4.4 Stokes $V$ was negative. This Stokes $V$ dependence of the apparent twist direction is common among all the investigated ARs and might suggest a cross talk between Stokes $V$ and Stokes $Q$ and $U$. Such a Stokes $V$ dependence could be caused by the leaking of some Stokes $V$ signal into the Stokes $Q$ and $U$ measurements. A clear correlation between the strength of the twist and the strength of the Stokes $V$ signal should therefore be observed. Fig. 4.8 displays a scatter plot between the Stokes $V$ amplitude in core pixels and their azimuthal twist for MFCs found in the plage of AR 10953. The correlation coefficient of this plot is merely 0.1 and suggests that the twist is independent of the Stokes $V$ amplitude. Another potential cross talk source that could induce the observed twist is from Stokes $I$. The azimuth angle of the observed magnetic field is calculated from the ratio between the Stokes $Q$ and $U$ measurements. In order to obtain a twist, the Stokes $Q$ and $U$ ratio within an absorption line must have a wavelength dependence. A cross talk from Stokes $I$ could induce such a wavelength dependence in the Stokes $Q$ and $U$ ratio due to the characteristic shape of the Stokes I profile. Fig. 4.9 displays a scatter plot between the Stokes $I$ line depth of core pixels and their twist. The correlation coefficient is only 0.2 , indicating that there is no relation between the Stokes $I$ line depth and the observed twist. The correlations shown in Figs. $4.8 \& 4.9$ were also performed by weighting the Stokes $V$ and $I$ signals with the linear polarisation for each pixels first. However, no improvement in the correlations coefficients was achieved. Although both Figs. $4.8 \& 4.9$ argue against the cross talk being the culprit for the observed twist in core pixels, we decided to perform 


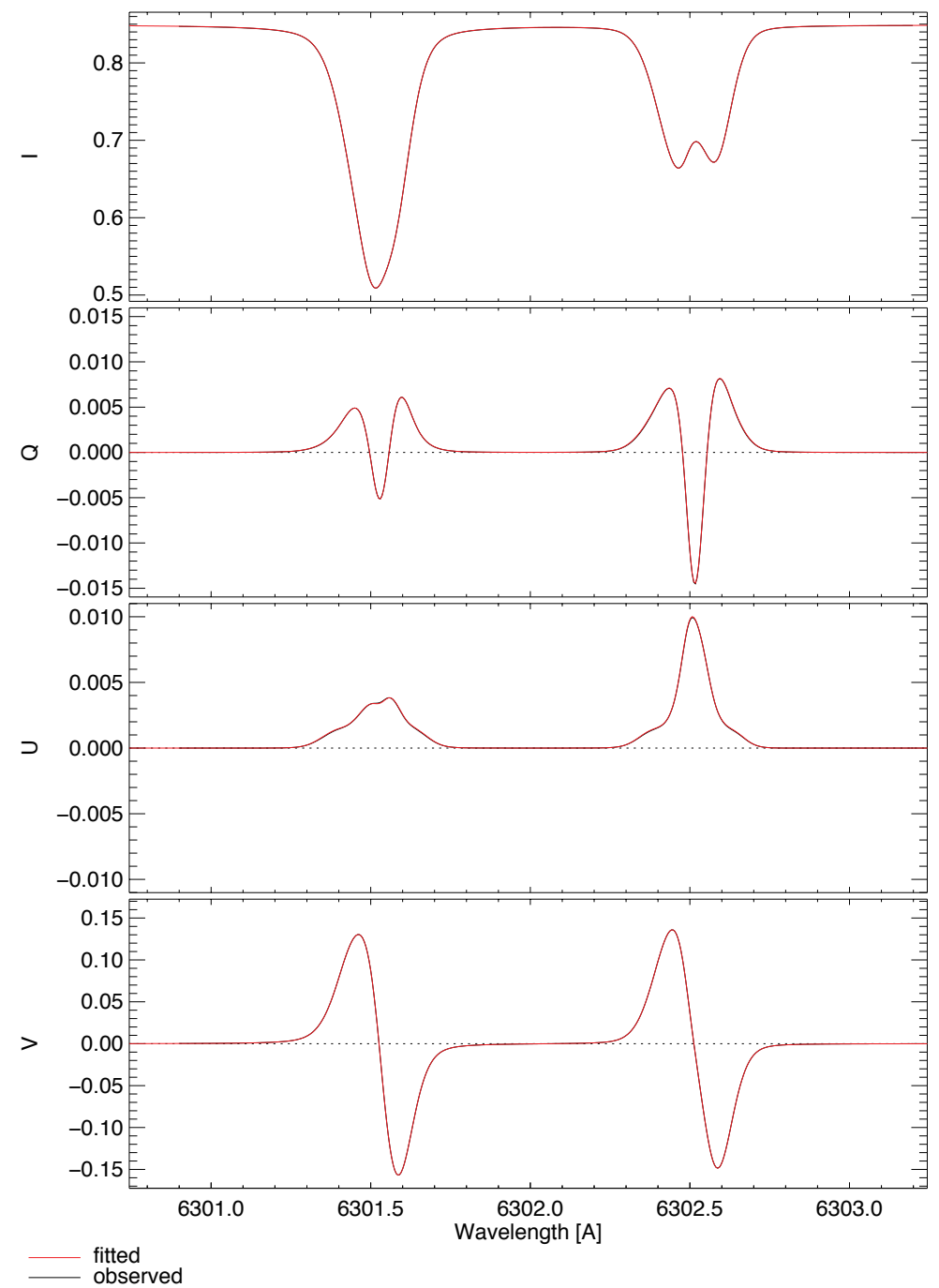

Figure 4.10: Black line shows the synthesized Stokes profiles of a plage-like atmosphere and red line depicts fitted spectra using SPINOR.

another test to conclusively rule out the possibility of a cross talk.

The SPINOR inversion code is based on the STOPRO routines (Solanki 1987), which perform a forward calculation. By providing STOPRO with a (solar) atmosphere, it can calculate the emergent Stokes profiles produced by the atmosphere assuming LTE. We proceeded by supplying STOPRO with a plage-like atmosphere based on the atmospheres calculated by SPINOR for AR 10953. The ideal emergent Stokes parameters of this plagelike atmosphere can be seen in Fig. 4.10. The black line shows the original synthesized spectra and the red line displays spectra fitted by SPINOR. The atmosphere employed here is already comparatively complex for it contains a gradient in the LOS velocity, the magnetic field and the microturbulence. The asymmetries in the emergent Stokes spectra are effected by these gradients. The atmospheric parameters at $\log (\tau)=0$ for this atmosphere were a magnetic field strength of $1600 \mathrm{G}$, an inclination of $20^{\circ}$, an azimuthal orientation of $0^{\circ}$, LOS velocity of $1.1 \mathrm{~km} / \mathrm{s}$, a microturbulence of $1.3 \mathrm{~km} / \mathrm{s}$ and a temperature of $6000 \mathrm{~K}$. For this particular atmosphere both the inclination and azimuth did not vary 


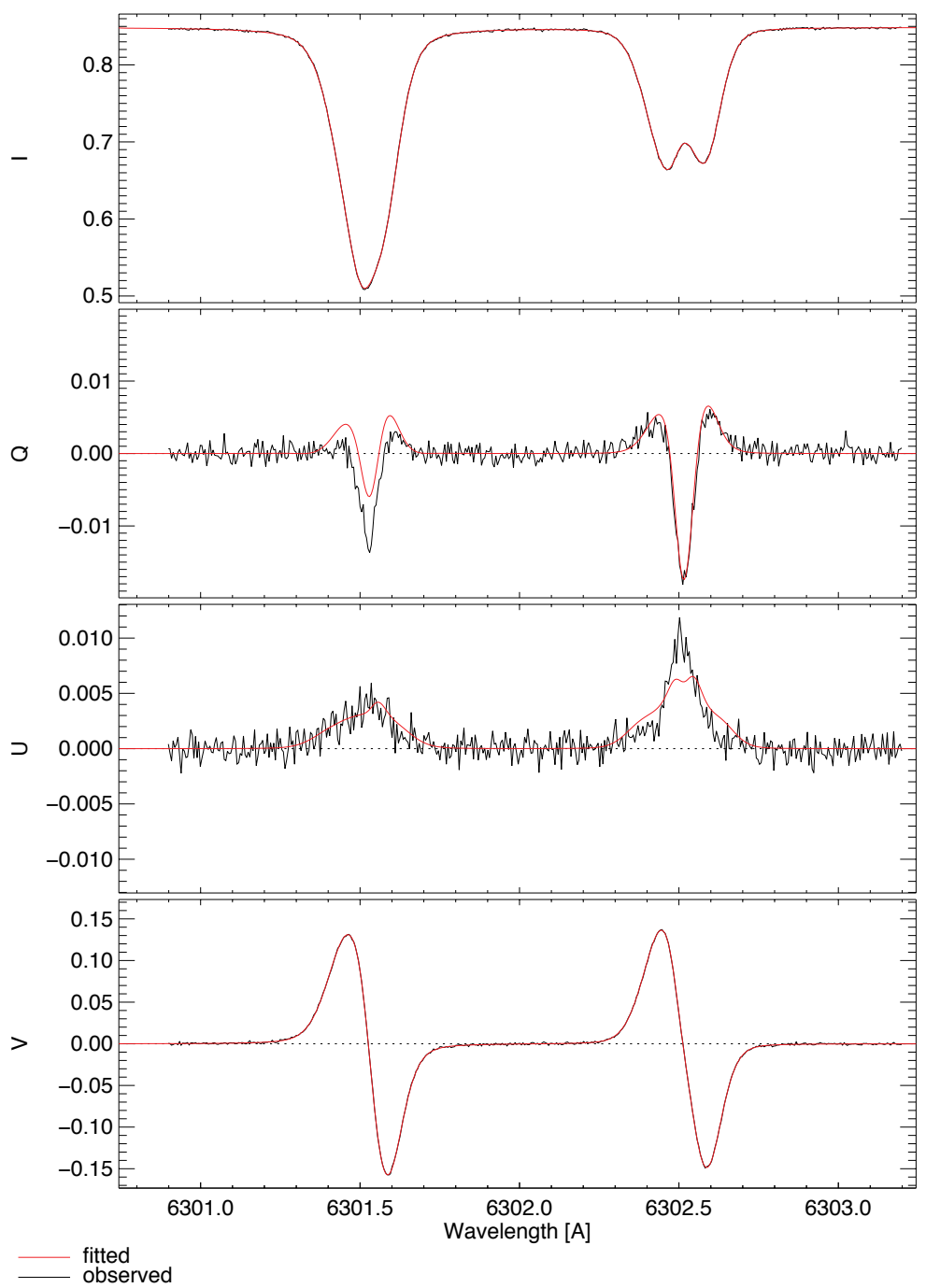

Figure 4.11: Black line shows the synthesized Stokes profiles of a plage like atmosphere with a cross talk from Stokes $I$ to Stokes $Q$. A noise of $1 \times 10^{-3} I_{c}$ has been added as well. The red line depicts the fitted spectra using SPINOR.

with $\log (\tau)$. Other plage-like atmospheres with a varying degree of complexity were tested as well, however, the results obtained from these atmospheres are similar to the ones presented in the remainder of this section and are, therefore, not discussed further. A common trait between all the tested atmospheres was the invariance of the azimuth with height.

The spectra in Fig. 4.10 were then progressively altered by adding various cross talks to either Stokes $Q$ or $U$, or both from Stokes $I$ or $V$. A noise of $1 \times 10^{-3} I_{c}$ was also added to every spectrum to simulate a Hinode SOT/SP observation. After a cross talk was added to the spectra they were inverted using SPINOR. The inversion setup including the placement of the $\log (\tau)$ nodes was identical to the setup used for Sects. $4.3 \& 4.4$, the only difference being that only one pixel was inverted at a time. By comparing the atmospheric parameters of the initial synthesized atmosphere with the atmosphere retrieved by SPINOR, we could reliably determine if and how much of a cross talk was necessary to produce a twist in the 
azimuth with height.

A large number of ARs studied in this Chapter showed average azimuth twists in their magnetic field of $30^{\circ}$, with one AR having an average of $60^{\circ}$. Therefore, it was decided that a cross talk must be able to reproduce a twist of at least $30^{\circ}$ in the magnetic field for it to be a possible source for the observed azimuth twist.

The analysis revealed that every type of cross talk is capable of producing the observed twist, be it Stokes $I$ into Stokes $Q$ and/or $U$, or Stokes $V$ into Stokes $Q$ and/or $U$. A cross talk between Stokes $Q$ and $U$ was also able to produce the required twist. An example of Stokes $I$ cross talk into Stokes $Q$ producing a $30^{\circ}$ twist can be seen in Fig. 4.11. The black line in all four spectra shows the artificial observation with the added cross talk and the red line shows the SPINOR fit to the spectra. The typical non-zero off set in the continuum produced by a Stokes $I$ cross talk was removed in Stokes $Q$ and $U$ prior to the inversion. A typical example of a Stokes $V$ cross talk into both Stokes $Q$ and $U$ is shown in Fig. 4.12, Again the added cross talk is able to produce a $30^{\circ}$ twist in the azimuth. The significance of the black and red spectra is identical to Fig. 4.11.

Figs. $4.11 \& 4.12$ confirm the assumption that a cross talk is capable to reproduce the observed azimuth twists, however, in both cases a large cross talk of $1 \%$ of the Stokes $V$ profile was necessary. The size of these cross talks is too large to be credible and in particular Fig. 4.12 displays clear Stokes $V$-like spectra in both Stokes $Q$ and $U$. Furthermore, the fits by SPINOR to the Stokes $Q$ and $U$ spectra are poor. If such a large Stokes $V$ cross talk would affect the observations then Fig. 4.8 should have shown a clear correlation. A $1 \%$ cross talk from Stokes $I$ is similarly unlikely since it would produce a large Stokes $Q$ or $U$ signal in all quiet Sun areas, which is not observed. Whilst we have shown that a cross talk can produce a twist in the magnetic field, the large cross talk required to achieve the necessary twist makes a cross talk as the source of the observed twist unlikely.

\subsection{Absorption line damping}

The widths of photospheric absorption lines such as the $6300 \AA$ line pair are affected by several physical processes, which cause them to broaden. When an observed spectrum is fitted by an inversion code such as SPINOR, it is important to take these processes into account accurately to retrieve reliable atmospheric parameters. By simultaneously fitting several absorption lines, a cross talk between these various line broadening mechanisms can be constrained. The most familiar broadening mechanisms, discussed so far, are the broadening due to the Zeeman effect as well as the microturbulence, both of which are free parameters within the inversion and thermal broadening.

However, there are further quantum mechanical effects, which can cause a line to broaden and in the case of solar absorption lines collisions between the emitting atom and neutral hydrogen is the dominant effect (de Jager and Neven 1970). The cross section, $\sigma$, determining the strength of this interaction needs to be supplied to the inversion. As a first approximation $\sigma$ can be estimated using a van der Waals interaction, however, for the transitions Fe I transitions used here, this approximation yields cross sections which are too small by a factor of 2 (Omara 1976). The cross sections used by SPINOR are taken from Anstee and O'Mara (1995); Barklem and O'Mara (1997); Barklem et al. (1998) 


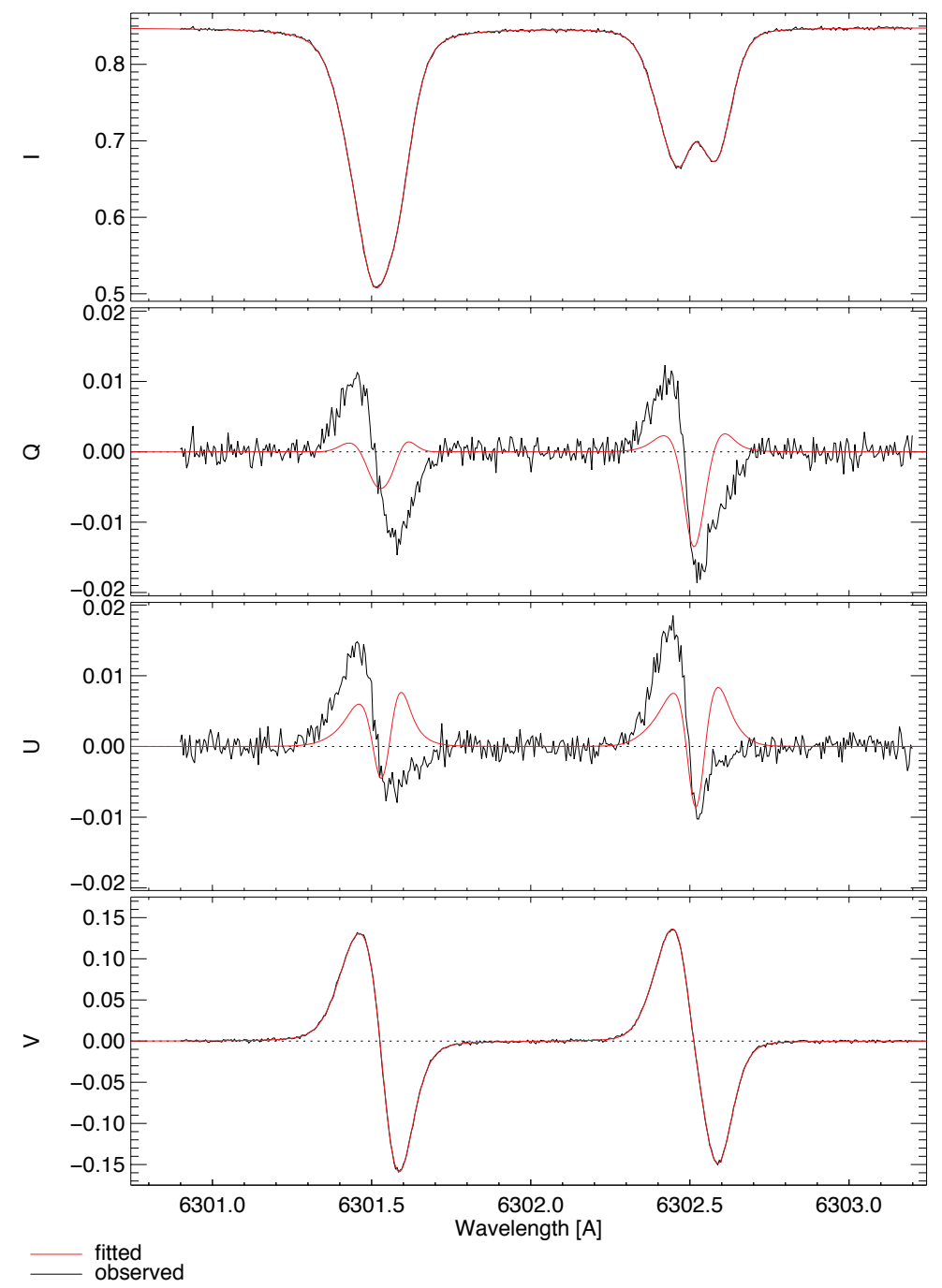

Figure 4.12: Black line shows the synthesized Stokes profiles of a plage like atmosphere with a cross talk from Stokes $V$ to Stokes $Q$ and $U$. A noise of $1 \times 10^{-3} I_{c}$ has been added as well. The red line depicts the fitted spectra using SPINOR.

who employed the more elaborate $\mathrm{ABO}$ theory characterizing the interaction. The cross sections were calculated for a relative collision velocity of $10^{4} \mathrm{~m} / \mathrm{s}$ and then scaled for other relative collision velocities using an exponent $\alpha$ in the form of $v^{-\alpha}$, where $v$ is the relative velocity. The broadening of the absorption lines by this process affect the wings of the lines disproportionately, which could in turn produce an artificial twist in the magnetic field. In the attempt to minimize $\chi^{2}$, SPINOR may use a free parameter such as the magnetic field to compensate for an erroneous line damping parameter.

For the two Fe I transitions studied here $\sigma$ takes a value of $847 a . u$. for $6302 \AA$ and $832 a . u$. for 6301 Å according to Anstee and O’Mara (1995); Barklem and O’Mara (1997); Barklem et al. (1998), where $a . u . \approx 2.8 \times 10^{-17} \mathrm{~cm}^{2}$, the Bohr radius squared. The red lines in Fig. 4.13 show a fit the to synthesized plage-like atmosphere, black lines, where $\sigma$ was changed by $100 \%$ from those given by (Anstee and O'Mara 1995; Barklem and O'Mara 1997; Barklem et al. 1998), with 6301A having $\sigma=100 a . u$. and $6302 \AA$ with $\sigma=1500 a . u$.. The 


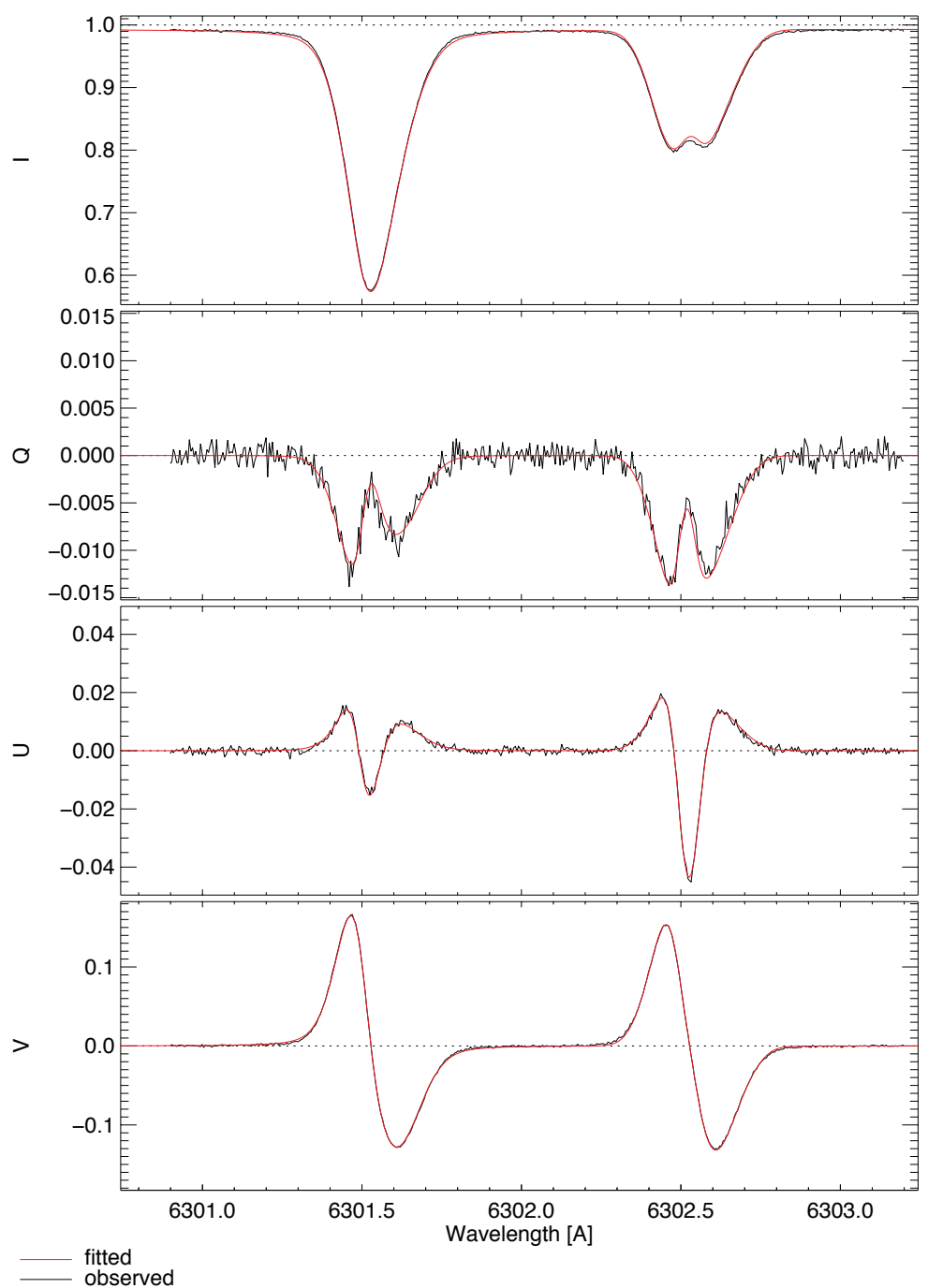

Figure 4.13: Black line shows the synthesized Stokes profiles of a plage like atmosphere with altered $\sigma$ for each line. A noise of $1 \times 10^{-3} I_{c}$ has been added as well. The red line depicts the fitted spectra using SPINOR.

twist in the magnetic field resulting from these changes in $\sigma$ amount to $10^{\circ}$, which is too small to account for the on average $30^{\circ}$ twist observed for some ARs. Furthermore, the implied uncertainty of $100 \%$ in $\sigma$ far outstrips the uncertainty of around $10 \%$ estimated by Anstee and O'Mara (1995) for their calculated cross sections. Other $\sigma$ values were also tested but generally yielded twists of $<10^{\circ}$ and are therefore not discussed further. Changes to $\alpha$ also did not reproduce the necessary twist.

\subsection{Other tests}

A series of further tests were carried out to discern a potential non solar origin of the observed twist in the magnetic fields. All of the tests described in this section were found to be incapable of producing the observed twist.

When a SPINOR inversion is started each free parameter is given an initial value from 
which to converge to the solution. A free parameter such as azimuth angle can be poorly constrained when some of the Stokes profiles are noise dominated. The returned solution for such a parameter after the inversion can then strongly depends on the initial values given. In order to test if the observed twist is merely the result of the initial input values each pixel was given the exact opposite twist as its input. It was found that such an initialized inversion was able to retrieve the clockwise twist direction described in sect. 4.3, demonstrating that the twist is well constrained.

The SPINOR inversion procedure performs a spatial deconvolution between neighbouring pixels using the Hinode SOT/SP PSF. However, the PSF employed is calculated from the instrument's specifications prior to launch and thus may not be identical to the actual PSF of SOT/SP. The sensitivity of the SPINOR solution to the employed PSF was tested by performing several inversion using PSFs with different amounts of added artificial defocus. The inversion results including the twist were negligibly affected by various the PSFs.

An earlier version of SPINOR did not perform a deconvolution of neighbouring pixels using the SOT/SP PSF but rather used a local straylight approximation (Orozco Suárez et al. 2007), whereby every profile had a straylight profile subtracted from it before it was inverted by SPINOR. It was found that the twist was also present in inversion results obtained using this version of SPINOR.

Furthermore, we tested if a change in the placement of the three $\log (\tau)$ nodes affected the observed twist. In particular, the placement of the highest node was changed, ranging between $\log (\tau)=-2$ to -2.5 . The lowest node was always kept at $\log (\tau)=0$ to obtain a good fit for the continuum. The middle node was kept at either $\log (\tau)=-0.9$ or -0.8 since the contribution functions for the $6300 \AA$ line pair have their maximum over this range. The observed twist displayed no dependence on the placement of the $\log (\tau)$ nodes over the considered $\log (\tau)$ ranges.

\subsection{Conclusion}

In this chapter we conducted an investigation into the apparent twist observed in magnetic fields in plage indicated by SPINOR inversion results. The inversion results analyzed in chapter 3 suggested that magnetic fields in plage in AR 10953 may posses a twist, but it was unclear whether this twist was conforming to Hale's helicity law or whether magnetic fields in other ARs inverted using SPINOR would show a similar behaviour.

Our initial investigation focussed on the magnetic fields in AR 10953 and we showed that both polarities of the magnetic field possess an overall clockwise twist, which is in good agreement with Hale's helicity law. Following Tiwari et al. (2009) we also calculated the global twists parameter $\alpha_{g}$ for the magnetic field in plage, which was in good agreement with $\alpha_{g}$ values obtained by Tiwari et al. (2009) for sunspots and also indicated an overall clockwise twist. Whilst the inversion results allows us to determine the twist in magnetic fields for each pixel individually by comparing different $\log (\tau)$ layers, the $\alpha_{g}$ parameter is calculated from the curl in the magnetic field in a single $\log (\tau)$ layer across all selected pixels. Based on these encouraging results we proceeded to analyze magnetic fields in other ARs.

We analyzed a further 19 ARs located in both the northern and southern hemispheres all of which were recorded by Hinode SOT/SP and subsequently inverted by SPINOR. All 
the ARs were found to have twisted magnetic fields located in areas of plage, even though the average twist varied between $10^{\circ}$ to $50^{\circ}$. However, all of the ARs displayed an overall clockwise twist and showed no reversal of the twist at the solar equator predicted by Hale's law and confirmed by Pevtsov et al. (1995); Abramenko et al. (1996) using magnetograms. This lack of agreement with Hale's helicity law strongly suggested that an instrumental or inversion based error might be responsible for the observed twists.

A series of tests were conducted in order to find a likely non-solar source for the observed twist, starting with an analysis examining a potential cross talk between the measured Stokes profiles. Even though no correlations were found between the twist and, both, the measured Stokes $I$ or $V$ amplitudes, we generated synthetic Stokes profiles of a plage-like atmosphere, containing a magnetic field with no twist, and added cross talks to Stokes $Q$ and/or $U$ from Stokes $I$ or $V$ before inverting the artificial profiles using SPINOR, to see if a cross talk could produce the necessary amount of twist. It was quickly realized that cross talks were indeed capable of producing a twist of $30^{\circ}$, but cross talks of $1 \%$ were considered too large to be credible, which ultimately excluded them as the source of the observed twists.

Another considered source of the twist was a potential error in the line damping parameter, $\sigma$, arising from collisions between the emitting atom and neutral hydrogen, which mainly affects the wings of absorption lines. The $\sigma$ values for both the transitions inverted by SPINOR were altered with the aim of producing the required twist in the magnetic field. Once again artificial Stokes profiles were generated, which contained no prior twist in the magnetic field to isolate the effect. Although a change in $\sigma$ could produce a twist in the magnetic field, only a twist of $10^{\circ}$ was achieved when both $\sigma$ 's were altered by $100 \%$ from the values normally employed by SPINOR. From this it was concluded that an error in $\sigma$ was unlikely to produce the observed twist, since the quoted uncertainties in $\sigma$ are only around $10 \%$.

Whilst several more tests were performed to uncover the source of the twist none were found to be credible, leaving open the possibility that the twist might be solar in origin after all. Nonetheless, the lack of agreement with Hale's helicity law make a solar interpretation of the twist challenging. 


\section{Conclusion \& Outlook}

In the preceding chapters we have studied various aspects and properties of small scale solar magnetic fields using Hinode SOT/SP observations.

The first investigation, described in Chapter 2, looked at magnetic fields found in internetwork regions in the disc centre of the quiet Sun. The stable, seeing-free data provided by SOT/SP allowed us to study the internetwork magnetic field over a period of six years, from November 2006 to May 2012. We were particularly interested in determining any cycle dependence in these fields. After considering instrumental degradations affecting the Stokes measurements, above all temperature variations aboard the satellite, the distribution of the LOS magnetic flux followed a power law with spectral index $\alpha=-1.82 \pm 0.02$, in agreement with previous publications and the variation of $\alpha$ for every year did not exceed a significance of $1 \sigma$. The SOT/SP data also allowed us to analyse magnetic fields orientated parallel to the solar surface via Stokes $Q$ and $U$ measurements, whose distribution also followed a power with $\alpha=-2.60 \pm 0.06$. Again, the yearly indices did not vary beyond a $1 \sigma$ significance. Furthermore, we carried out a patch size analysis. Both, the LOS magnetic flux and the linear polarisation distributions of their magnetic patches follow power laws with indices, $\alpha=-2.20 \pm 0.05$ and $\alpha=-3.03 \pm 0.08$, respectively, indicating that the magnetic flux increases rapidly with patch size. These results show the internetwork magnetic fields to be invariant over the considered time period, suggesting that independent local dynamo action may be the main source for these fields.

An immediate extension of this investigation would be the addition of quiet Sun disc centre SOT/SP scans recorded after May 2012, to determine if the stated conclusion holds over longer time periods. The motivation for such an extension is deeper than mere curiosity when comparing this study to investigations analysing the variation of sunspot numbers with time, with reliable measurements starting in the 1600s. Starting with the considerable variation between solar cycles, and more longer secular variations of the Sun's magnetic fields to the extended periods of grand minima such as the Maunder minimum, the investigation conducted in Chapter 2 is, both, insufficient and yet indispensable, when considering that it suggests that local near-surface dynamos could render the Sun magnetically active even during a grand minimum. Another extension to this study could be a combination of SOT/SP results with SDO/HMI measurements, with the aims of increasing the field-of-view from the relatively limited 30 " $\times 240$ " currently employed and increasing the temporal resolution from currently one measurement per month to at least a daily measurement of the internetwork magnetic fields. Furthermore, SDO/HMI could be used to study the emergence rate of magnetic flux similar to the study performed by Hagenaar et al. (2003). The emergence rate reported by Thornton and Parnell (2011), using SOT/NFI data, could be extended to cover the entire solar disc and a longer time scale, with the aim of potentially verifying the somewhat lower emergence rate published by Guglielmino 
et al. (2012). However, it remains to be determined whether the lower spatial resolution of $0 . " 5$ and spectral resolution of HMI will be sufficient to study the quiet Sun internetwork in the necessary detail.

In the second investigation, detailed in Chapter 3, we analysed the properties of magnetic fields found in plage in AR 10953. The SOT/SP spectra were inverted using an extended version of the SPINOR code, which performed a spatially coupled $2 \mathrm{D}$ inversion. By allowing gradients in the atmospheric parameters, such as LOS velocity and magnetic field gradients, and a proper treatment of the instrument's point-spread-function, we were able to fit the observed Stokes spectra using only one atmospheric component per pixel, thereby setting the filling factor equal to one. The results of the inversion were then employed to examine some of the properties of the $k G$ magnetic features found in plage. The inversion results were able to confirm several previously discovered properties that used the $6300 \AA$ line pair, such as an average field strength of $1490 \mathrm{G}$, with typical inclinations of $10^{\circ}-15^{\circ}$ and only weak LOS downflows of $0.37 \mathrm{~km} / \mathrm{s}$ on average. With the inclusion of gradients we were, in addition, able to analyse the height dependence of the atmospheric parameters. In particular we studied the expansion of the magnetic features with height and compared it to two empirical zeroth order thin flux-tube models. Not only could the expansion be described reasonably well by such a model, we were also able to show that of the two models, the plage flux tube model resembled the observed structures better than the network flux tube model. This was apparent the most in the temperature stratifications. Furthermore, each magnetic feature was surrounded by a ring of strong downflows typically $1-3 \mathrm{~km} / \mathrm{s}$. Within these downflows small patches bearing the opposite magnetic polarity to the main feature were found. These patches contained magnetic fields with apparent strengths barely higher than $100 \mathrm{G}$ and were hidden below stronger fields forming the canopy of the main feature. Such weak opposite polarity patches are predicted by MHD simulations but so far had eluded observational confirmation. The azimuthal orientation of the magnetic fields is in general isotropic and only fields found in the direct vicinity of another magnetic feature, such as a sunspot, can display highly inclined magnetic fields and deformed canopies. Furthermore, an increased microturbulence was observed both in and especially around magnetic features when compared to the quiet Sun possibly hinting at waves, or unresolved velocities.

Chapter 4 presents a followup study of the investigation described in Chapter 3. The azimuthal orientations of the magnetic fields found in plage in AR 10953 were not constant with height and implied the presence of a twist in the magnetic field. We sought to determine if this twist is solar in origin or an artifact of the inversion or SOT/SP. To this end we examined several plage regions in other ARs across the solar disc recorded by SOT/SP using the same inversion method. All active regions displayed a twist in the azimuth with height, however, a hemispherical dependence in the twist direction, in accordance with Hale's helicity law, could not be established. This, in turn, led to several tests to determine a possible instrumental or inversion-based cause for the observed twists. Among the tested scenarios were various cross talks between the Stokes parameters as well as uncertainties in the line-damping parameters used by the inversion. However, no instrumental or inversion based effect could be found to conclusively explain the observed twists.

There are several ways in which the work described in Chapters $3 \& 4$ could be extended. First and foremost among those should be the completion of the investigation started in Chapter 4, since uncovering the source of the observed twists would, in any case, result 
in a greater understanding of the employed inversion method and its reliability. Another immediate extension could come in the form of turning the azimuth ambiguity removal method, written specifically for analysing the magnetic field vector in plage in AR 10953, into a general tool accompanying SPINOR inversions. Other potential future investigations could be a reexamination of the filament in AR 10953 using the extended SPINOR code employed here, with the aim of extending upon the ME-based investigations of this filament by Okamoto et al. (2008, 2009). Of particular interest would be its emergence and rise through the photosphere as well as the orientation of its magnetic fields during this process. With the extended SPINOR inversion method it may be possible to conclusively identify this filament as an emerging twisted flux rope as well as study its internal structure as it rises though the photosphere. The database of ARs already used in Chapter 4 could also be used to study other aspects of magnetic fields in plage, in particular the morphology and its relation to other physical parameters. The relationship between magnetic field strength and continuum intensity (Kobel et al. 2011) and the comparison between observations and MHD simulations (Danilovic et al.2013) could be reexamined, given our improved treated of local straylight, which allows us to fit profiles using only a single atmospheric component. This investigation could then also be extended to a centre-to-limb investigation. Other investigations could aim at examining the dependence between the size or flux density of magnetic features with the line-of-sight velocities to ascertain whether the ring of downflows surrounding a feature (see Chapter 3) is related its size, as was indicated by Cho et al. (2010) by analysing pores. A similar analysis could be performed to determine a possible relationship between the microtubulence and the size or flux density of a feature, given the clear association of high microturbulence and magnetic features in our inversions. 



\section{Bibliography}

Abramenko, V. I., Wang, T., Yurchishin, V. B., 1996, Analysis of Electric Current Helicity in Active Regions on the Basis of Vector Magnetograms, Sol. Phys., 168, 75-89

Anstee, S. D., O’Mara, B. J., 1995, Width cross-sections for collisional broadening of s-p and p-s transitions by atomic hydrogen, MNRAS, 276, 859-866

Asensio Ramos, A., 2009, Evidence for Quasi-Isotropic Magnetic Fields from Hinode Quiet-Sun Observations, ApJ, 701, 1032-1043

Attie, R., Innes, D. E., Potts, H. E., 2009, Evidence of photospheric vortex flows at supergranular junctions observed by FG/SOT (Hinode), A\&A, 493, L13-L16

Auer, L. H., House, L. L., Heasley, J. N., 1977, The determination of vector magnetic fields from Stokes profiles, Sol. Phys., 55, 47-61

Babcock, H. W., Babcock, H. D., 1955, The Sun's Magnetic Field, 1952-1954., ApJ, 121, 349

Balmaceda, L., Vargas Domínguez, S., Palacios, J., Cabello, I., Domingo, V., 2010, Evidence of small-scale magnetic concentrations dragged by vortex motion of solar photospheric plasma, A\&A, 513, L6

Barklem, P. S., O’Mara, B. J., 1997, The broadening of p-d and d-p transitions by collisions with neutral hydrogen atoms, MNRAS, 290, 102-106

Barklem, P. S., O’Mara, B. J., Ross, J. E., 1998, The broadening of d-f and f-d transitions by collisions with neutral hydrogen atoms, MNRAS, 296, 1057-1060

Barthol, P., Gandorfer, A., Solanki, S. K., Schüssler, M., Chares, B., Curdt, W., Deutsch, W., Feller, A., Germerott, D., Grauf, B., Heerlein, K., Hirzberger, J., Kolleck, M., Meller, R., Müller, R., Riethmüller, T. L., Tomasch, G., Knölker, M., Lites, B. W., Card, G., Elmore, D., Fox, J., Lecinski, A., Nelson, P., Summers, R., Watt, A., Martínez Pillet, V., Bonet, J. A., Schmidt, W., Berkefeld, T., Title, A. M., Domingo, V., Gasent Blesa, J. L., Del Toro Iniesta, J. C., López Jiménez, A., Álvarez-Herrero, A., Sabau-Graziati, L., Widani, C., Haberler, P., Härtel, K., Kampf, D., Levin, T., Pérez Grande, I., Sanz-Andrés, A., Schmidt, E., 2011, The Sunrise Mission, Sol. Phys., 268, 1-34

Beckers, J. M., 1981, Dynamics of the solar photosphere, NASA Special Publication, 450, 11-64 
Beckers, J. M., Bridges, C. A., Gilliam, L. B., 1976, A high resolution spectral atlas of the solar irradiance from 380 to 700 nanometers. Volume 2: Graphical form, Air Force Geophysics Laboratory

Bellot Rubio, L. R., Collados, M., 2003, Understanding internetwork magnetic fields as determined from visible and infrared spectral lines, A\&A, 406, 357-362

Bellot Rubio, L. R., Ruiz Cobo, B., Collados, M., 1997, Flux-Tube Model Atmospheres and Stokes V Zero-crossing Wavelengths, ApJ, 478, L45, arXiv:astro-ph/9701059

Bellot Rubio, L. R., Ruiz Cobo, B., Collados, M., 1998, An Hermitian Method for the Solution of Polarized Radiative Transfer Problems, ApJ, 506, 805-817

Bellot Rubio, L. R., Ruiz Cobo, B., Collados, M., 2000, Structure of Plage Flux Tubes from the Inversion of Stokes Spectra. I. Spatially Averaged Stokes I and V Profiles, ApJ, $535,489-500$

Berger, T. E., Title, A. M., 1996, On the Dynamics of Small-Scale Solar Magnetic Elements, ApJ, 463, 365

Berger, T. E., Title, A. M., 2001, On the Relation of G-Band Bright Points to the Photospheric Magnetic Field, ApJ, 553, 449-469

Berger, T. E., Rouppe van der Voort, L. H. M., Löfdahl, M. G., Carlsson, M., Fossum, A., Hansteen, V. H., Marthinussen, E., Title, A., Scharmer, G., 2004, Solar magnetic elements at 0.1 arcsec resolution. General appearance and magnetic structure, A\&A, 428, 613-628

Bernasconi, P. N., Keller, C. U., Povel, H. P., Stenflo, J. O., 1995, Direct measurements of flux tube inclinations in solar plages., A\&A, 302, 533

Bommier, V., Derouich, M., Landi Degl'Innocenti, E., Molodij, G., Sahal-Bréchot, S., 2005, Interpretation of second solar spectrum observations of the Sr I $4607 \AA$ line in a quiet region: Turbulent magnetic field strength determination, A\&A, 432, 295-305

Bonet, J. A., Márquez, I., Sánchez Almeida, J., Cabello, I., Domingo, V., 2008, Convectively Driven Vortex Flows in the Sun, ApJ, 687, L131-L134

Bonet, J. A., Márquez, I., Sánchez Almeida, J., Palacios, J., Martínez Pillet, V., Solanki, S. K., del Toro Iniesta, J. C., Domingo, V., Berkefeld, T., Schmidt, W., Gandorfer, A., Barthol, P., Knölker, M., 2010, SUNRISE/IMaX Observations of Convectively Driven Vortex Flows in the Sun, ApJ, 723, L139-L143

Borrero, J. M., Ichimoto, K., 2011, Magnetic Structure of Sunspots, Living Reviews in Solar Physics, 8, 4

Borrero, J. M., Kobel, P., 2011, Inferring the magnetic field vector in the quiet Sun. I. Photon noise and selection criteria, A\&A, 527, A29

Borrero, J. M., Kobel, P., 2012, Inferring the magnetic field vector in the quiet Sun. II. Interpreting results from the inversion of Stokes profiles, A\&A, 547, A89 
Borrero, J. M., Kobel, P., 2013, Inferring the magnetic field vector in the quiet Sun. III. Disk variation of the Stokes profiles and isotropism of the magnetic field, A\&A, 550, A98

Brandt, P. N., Scharmer, G. B., Ferguson, S., Shine, R. A., Tarbell, T. D., 1988, Vortex flow in the solar photosphere, Nature, 335, 238-240

Briand, C., Solanki, S. K., 1998, Velocity fields below the magnetic canopy of solar flux tubes: evidence for high-speed downflows?, A\&A, 330, 1160-1168

Bruls, J. H. M. J., Solanki, S. K., 1995, Infrared lines as probes of solar magnetic features. IX. MgI $12 \mu \mathrm{m}$ diagnostics of solar plage., A\&A, 293, 240-251

Buente, M., Solanki, S. K., Steiner, O., 1993, Centre-to-limb variation of the Stokes V asymmetry in solar magnetic flux tubes, A\&A, 268, 736-748

Carlsson, M., 1986, A computer program for solving multi-level non-LTE radiative transferproblems in moving or static atmospheres., Uppsala Astronomical Observatory Reports, 33

Cattaneo, F., 1999, On the Origin of Magnetic Fields in the Quiet Photosphere, ApJ, 515, L39-L42

Charbonneau, P., 2010, Dynamo models of the solar cycle, Living Reviews in Solar Physics, 7

Cho, K.-S., Bong, S.-C., Chae, J., Kim, Y.-H., Park, Y.-D., 2010, Tiny Pores Observed by Hinode/Solar Optical Telescope, ApJ, 723, 440-448

Choudhuri, A. R., Chatterjee, P., Nandy, D., 2004, Helicity of Solar Active Regions from a Dynamo Model, ApJ, 615, L57-L60

Culhane, L., Harra, L. K., Baker, D., van Driel-Gesztelyi, L., Sun, J., Doschek, G. A., Brooks, D. H., Lundquist, L. L., Kamio, S., Young, P. R., Hansteen, V. H., 2007, Hinode EUV Study of Jets in the Sun's South Polar Corona, PASJ, 59, 751

Danilovic, S., Gandorfer, A., Lagg, A., Schüssler, M., Solanki, S. K., Vögler, A., Katsukawa, Y., Tsuneta, S., 2008, The intensity contrast of solar granulation: comparing Hinode SP results with MHD simulations, A\&A, 484, L17-L20

Danilovic, S., Beeck, B., Pietarila, A., Schüssler, M., Solanki, S. K., Martínez Pillet, V., Bonet, J. A., del Toro Iniesta, J. C., Domingo, V., Barthol, P., Berkefeld, T., Gandorfer, A., Knölker, M., Schmidt, W., Title, A. M., 2010a, Transverse Component of the Magnetic Field in the Solar Photosphere Observed by SUNRISE, ApJ, 723, L149-L153

Danilovic, S., Schüssler, M., Solanki, S. K., 2010b, Probing quiet Sun magnetism using MURaM simulations and Hinode/SP results: support for a local dynamo, A\&A, 513, A1

Danilovic, S., Schüssler, M., Solanki, S. K., 2010c, Magnetic field intensification: comparison of 3D MHD simulations with Hinode/SP results, A\&A, 509, A76 
Danilovic, S., Röhrbein, D., Cameron, R. H., Schüssler, M., 2013, On the relation between continuum brightness and magnetic field in solar active regions, A\&A, 550, A118

de Jager, C., Neven, L., 1970, Damping Constants for Infrared Fraunhofer Lines, Sol. Phys., $11,3-10$

de Wijn, A. G., Rutten, R. J., Haverkamp, E. M. W. P., Sütterlin, P., 2005, DOT tomography of the solar atmosphere. IV. Magnetic patches in internetwork areas, A\&A, 441, 11831190

de Wijn, A. G., Stenflo, J. O., Solanki, S. K., Tsuneta, S., 2009, Small-Scale Solar Magnetic Fields, Space Sci. Rev., 144, 275-315

Defouw, R. J., 1976, Wave propagation along a magnetic tube, ApJ, 209, 266-269

Deinzer, W., Hensler, G., Schussler, M., Weisshaar, E., 1984, Model Calculations of Magnetic Flux Tubes - Part Two - Stationary Results for Solar Magnetic Elements, A\&A, 139, 435

del Toro Iniesta, J. C., 2003, Introduction to Spectropolarimetry, Cambridge University Press

Del Toro Iniesta, J. C., Ruiz Cobo, B., 1996, Stokes Profiles Inversion Techniques, Sol. Phys., 164, 169-182

Deubner, F.-L., Fleck, B., 1990, Dynamics of the solar atmosphere. III - Cell-network distinctions of chromospheric oscillations, A\&A, 228, 506-512

Domingo, V., Ermolli, I., Fox, P., Fröhlich, C., Haberreiter, M., Krivova, N., Kopp, G., Schmutz, W., Solanki, S. K., Spruit, H. C., Unruh, Y., Vögler, A., 2009, Solar Surface Magnetism and Irradiance on Time Scales from Days to the 11-Year Cycle, Space Sci. Rev., 145, 337-380

Domínguez Cerdeña, I., Kneer, F., Sánchez Almeida, J., 2003, Quiet-Sun Magnetic Fields at High Spatial Resolution, ApJ, 582, L55-L58

Domínguez Cerdeña, I., Sánchez Almeida, J., Kneer, F., 2006, The Distribution of Quiet Sun Magnetic Field Strengths from 0 to 1800 G, ApJ, 636, 496-509

Dravins, D., Lindegren, L., Nordlund, A., 1981, Solar granulation - Influence of convection on spectral line asymmetries and wavelength shifts, A\&A, 96, 345-364

Dunn, R. B., Zirker, J. B., 1973, The Solar Filigree, Sol. Phys., 33, 281-304

Eddy, J. A., 1976, The Maunder Minimum, Science, 192, 1189-1202

Faurobert-Scholl, M., 1996, Diagnostics with the Hanle Effect, Sol. Phys., 164, 79-90

Ferriz-Mas, A., Schuessler, M., Anton, V., 1989, Dynamics of magnetic flux concentrations - The second-order thin flux tube approximation, A\&A, 210, 425-432 
Fleck, B., Schmitz, F., 1991, The 3-min oscillations of the solar chromosphere - A basic physical effect?, A\&A, 250, 235-244

Frazier, E. N., 1971, Multi-Channel Magnetograph Observations. III: Faculae, Sol. Phys., $21,42-53$

Frazier, E. N., Stenflo, J. O., 1972, On the Small-Scale Structure of Solar Magnetic Fields, Sol. Phys., 27, 330-346

Fröhlich, C., 2009, Evidence of a long-term trend in total solar irradiance, A\&A, 501, L27

Frutiger, C., Solanki, S. K., 1998, Do solar magnetic elements harbor downflows?, A\&A, 336, L65-L68

Frutiger, C., Solanki, S. K., 2001, Empirical models of solar magnetic flux-tubes and their non-magnetic surroundings, A\&A, 369, 646-659

Frutiger, C., Solanki, S. K., Fligge, M., Bruls, J. H. M. J., 2000, Properties of the solar granulation obtained from the inversion of low spatial resolution spectra, A\&A, 358, 1109-1121

Gingerich, O., Noyes, R. W., Kalkofen, W., Cuny, Y., 1971, The Harvard-Smithsonian reference atmosphere, Sol. Phys., 18, 347-365

Giovanelli, R. G., 1982, Sunspot geometry and pressure balance, Sol. Phys., 80, 21-31

Giovanelli, R. G., Slaughter, C., 1978, Motions in solar magnetic tubes. I - The downflow, Sol. Phys., 57, 255-260

Golub, L., Deluca, E., Austin, G., Bookbinder, J., Caldwell, D., Cheimets, P., Cirtain, J., Cosmo, M., Reid, P., Sette, A., Weber, M., Sakao, T., Kano, R., Shibasaki, K., Hara, H., Tsuneta, S., Kumagai, K., Tamura, T., Shimojo, M., McCracken, J., Carpenter, J., Haight, H., Siler, R., Wright, E., Tucker, J., Rutledge, H., Barbera, M., Peres, G., Varisco, S., 2007, The X-Ray Telescope (XRT) for the Hinode Mission, Sol. Phys., 243, 63-86

Grossmann-Doerth, U., Schuessler, M., Solanki, S. K., 1988, Unshifted, asymmetric Stokes V-profiles - Possible solution of a riddle, A\&A, 206, L37-L39

Grossmann-Doerth, U., Schuessler, M., Solanki, S. K., 1989, Stokes V asymmetry and shift of spectral lines, A\&A, 221, 338-341

Grossmann-Doerth, U., Schuessler, M., Solanki, S. K., 1991, The effect of non-linear oscillations in magnetic flux tubes on Stokes V asymmetry, A\&A, 249, 239-242

Grossmann-Doerth, U., Knoelker, M., Schuessler, M., Solanki, S. K., 1994, The deep layers of solar magnetic elements, A\&A, 285, 648-654

Grossmann-Doerth, U., Schuessler, M., Steiner, O., 1998, Convective intensification of solar surface magnetic fields: results of numerical experiments, A\&A, 337, 928-939 
Guglielmino, S. L., Martínez Pillet, V., Bonet, J. A., del Toro Iniesta, J. C., Bellot Rubio, L. R., Solanki, S. K., Schmidt, W., Gandorfer, A., Barthol, P., Knölker, M., 2012, The Frontier between Small-scale Bipoles and Ephemeral Regions in the Solar Photosphere: Emergence and Decay of an Intermediate-scale Bipole Observed with SUNRISE/IMaX, ApJ, 745, 160, 1110.1405

Hagenaar, H. J., 2001, Ephemeral Regions on a Sequence of Full-Disk Michelson Doppler Imager Magnetograms, ApJ, 555, 448-461

Hagenaar, H. J., Schrijver, C. J., Title, A. M., 2003, The Properties of Small Magnetic Regions on the Solar Surface and the Implications for the Solar Dynamo(s), ApJ, 584, 1107

Hale, G. E., 1908, On the Probable Existence of a Magnetic Field in Sun-Spots, ApJ, 28, 315

Hale, G. E., 1927, The Fields of Force in the Atmosphere of the Sun, Nature, 119, 708-714

Harvey, J. W., Branston, D., Henney, C. J., Keller, C. U., SOLIS and GONG Teams, 2007, Seething Horizontal Magnetic Fields in the Quiet Solar Photosphere, ApJ, 659, L177-L180

Harvey, K. L., 1993, Magnetic Bipoles on the Sun, Ph.D. thesis, Univ. Utrecht, (1993)

Harvey, K. L., Harvey, J. W., Martin, S. F., 1975, Ephemeral active regions in 1970 and 1973, Sol. Phys., 40, 87-102

Hathaway, D. H., 2010, The solar cycle, Living Reviews in Solar Physics, 7

Holweger, H., Gehlsen, M., Ruland, F., 1978, Spatially-averaged Properties of the Photospheric Velocity Field, A\&A, 70, 537

Howard, R., Stenflo, J. O., 1972, On the Filamentary Nature of Solar Magnetic Fields, Sol. Phys., 22, 402-417

Ichimoto, K., Lites, B., Elmore, D., Suematsu, Y., Tsuneta, S., Katsukawa, Y., Shimizu, T., Shine, R., Tarbell, T., Title, A., Kiyohara, J., Shinoda, K., Card, G., Lecinski, A., Streander, K., Nakagiri, M., Miyashita, M., Noguchi, M., Hoffmann, C., Cruz, T., 2008, Polarization Calibration of the Solar Optical Telescope onboard Hinode, Sol. Phys., 249, 233-261

Iida, Y., Hagenaar, H. J., Yokoyama, T., 2012, Detection of Flux Emergence, Splitting, Merging, and Cancellation of Network Field. I. Splitting and Merging, ApJ, 752, 149

Ishikawa, R., Tsuneta, S., 2009, Comparison of transient horizontal magnetic fields in a plage region and in the quiet Sun, A\&A, 495, 607

Ishikawa, R., Tsuneta, S., 2010, Spatial and Temporal Distributions of Transient Horizontal Magnetic Fields with Deep Exposure, ApJ, 718, L171-L175 
Ishikawa, R., Tsuneta, S., 2011, The Relationship between Vertical and Horizontal Magnetic Fields in the Quiet Sun, ApJ, 735, 74

Ishikawa, R., Tsuneta, S., Ichimoto, K., Isobe, H., Katsukawa, Y., Lites, B. W., Nagata, S., Shimizu, T., Shine, R. A., Suematsu, Y., Tarbell, T. D., Title, A. M., 2008, Transient horizontal magnetic fields in solar plage regions, A\&A, 481, L25-L28

Ito, H., Tsuneta, S., Shiota, D., Tokumaru, M., Fujiki, K., 2010, Is the Polar Region Different from the Quiet Region of the Sun?, ApJ, 719, 131

Jafarzadeh, S., Solanki, S. K., Feller, A., Lagg, A., Pietarila, A., Danilovic, S., Riethmüller, T. L., Martínez Pillet, V., 2013, Structure and dynamics of isolated internetwork Ca II H bright points observed by SUNRISE, A\&A, 549, A116, 1211.4836

Jefferies, J., Lites, B. W., Skumanich, A., 1989, Transfer of line radiation in a magnetic field, ApJ, 343, 920-935

Jin, C. L., Wang, J. X., 2012, The Latitude Distribution of Small-scale Magnetic Elements in Solar Cycle 23, ApJ, 745, 39

Keller, C. U., 1992, Resolution of magnetic flux tubes on the sun, Nature, 359, 307

Keller, C. U., Stenflo, J. O., Solanki, S. K., Tarbell, T. D., Title, A. M., 1990, Solar magnetic field strength determinations from high spatial resolution filtergrams, A\&A, 236, 250-255

Keys, P. H., Mathioudakis, M., Jess, D. B., Shelyag, S., Crockett, P. J., Christian, D. J., Keenan, F. P., 2011, The Velocity Distribution of Solar Photospheric Magnetic Bright Points, ApJ, 740, L40

Khomenko, E. V., Collados, M., Solanki, S. K., Lagg, A., Trujillo Bueno, J., 2003, Quiet-Sun inter-network magnetic fields observed in the infrared, A\&A, 408, 1115

Khomenko, E. V., Martínez González, M. J., Collados, M., Vögler, A., Solanki, S. K., Ruiz Cobo, B., Beck, C., 2005, Magnetic flux in the internetwork quiet Sun, A\&A, 436, L27-L30

Kleint, L., Berdyugina, S. V., Shapiro, A. I., Bianda, M., 2010, Solar turbulent magnetic fields: surprisingly homogeneous distribution during the solar minimum, A\&A, 524, A37

Kobel, P., Hirzberger, J., Solanki, S. K., Gandorfer, A., Zakharov, V., 2009, Discriminant analysis of solar bright points and faculae. I. Classification method and center-to-limb distribution, A\&A, 502, 303-314

Kobel, P., Solanki, S. K., Borrero, J. M., 2011, The continuum intensity as a function of magnetic field. I. Active region and quiet Sun magnetic elements, A\&A, 531, A112

Kobel, P., Solanki, S. K., Borrero, J. M., 2012, The continuum intensity as a function of magnetic field. II. Local magnetic flux and convective flows, A\&A, 542, A96 
Kosugi, T., Matsuzaki, K., Sakao, T., Shimizu, T., Sone, Y., Tachikawa, S., Hashimoto, T., Minesugi, K., Ohnishi, A., Yamada, T., Tsuneta, S., Hara, H., Ichimoto, K., Suematsu, Y., Shimojo, M., Watanabe, T., Shimada, S., Davis, J. M., Hill, L. D., Owens, J. K., Title, A. M., Culhane, J. L., Harra, L. K., Doschek, G. A., Golub, L., 2007, The Hinode (Solar-B) Mission: An Overview, Sol. Phys., 243, 3-17

Krivova, N. A., Solanki, S. K., Fligge, M., Unruh, Y. C., 2003, Reconstruction of solar irradiance variations in cycle 23: Is solar surface magnetism the cause?, A\&A, 399, L1-L4

Krivova, N. A., Balmaceda, L., Solanki, S. K., 2007, Reconstruction of solar total irradiance since 1700 from the surface magnetic flux, A\&A, 467, 335-346

Kuckein, C., Martínez Pillet, V., Centeno, R., 2012, An active region filament studied simultaneously in the chromosphere and photosphere. I. Magnetic structure, A\&A, 539, A131

Lagg, A., Solanki, S. K., Riethmüller, T. L., Martínez Pillet, V., Schüssler, M., Hirzberger, J., Feller, A., Borrero, J. M., Schmidt, W., del Toro Iniesta, J. C., Bonet, J. A., Barthol, P., Berkefeld, T., Domingo, V., Gandorfer, A., Knölker, M., Title, A. M., 2010, Fully Resolved Quiet-Sun Magnetic flux Tube Observed with the SUNRISE/IMAX Instrument, ApJ, 723, L164-L168

Langangen, Ø., Carlsson, M., Rouppe van der Voort, L., Stein, R. F., 2007, Velocities Measured in Small-Scale Solar Magnetic Elements, ApJ, 655, 615-623

Leighton, R. B., Noyes, R. W., Simon, G. W., 1962, Velocity Fields in the Solar Atmosphere. I. Preliminary Report., ApJ, 135, 474

Leka, K. D., Barnes, G., Crouch, A. D., Metcalf, T. R., Gary, G. A., Jing, J., Liu, Y., 2009, Resolving the 180degree Ambiguity in Solar Vector Magnetic Field Data: Evaluating the Effects of Noise, Spatial Resolution, and Method Assumptions, Sol. Phys., 260, $83-108$

Lin, H., 1995, On the Distribution of the Solar Magnetic Fields, ApJ, 446, 421

Lin, H., Rimmele, T., 1999, The Granular Magnetic Fields of the Quiet Sun, ApJ, 514, $448-455$

Lites, B. W., 2002, Characterization of Magnetic Flux in the Quiet Sun, ApJ, 573, 431-444

Lites, B. W., 2011, Hinode Observations Suggesting the Presence of a Local Small-scale Turbulent Dynamo, ApJ, 737, 52

Lites, B. W., Ichimoto, K., 2013, The SP_PREP Data Preparation Package for the Hinode Spectro-Polarimeter, Sol. Phys., 283, 601-629

Lites, B. W., Skumanich, A., 1990, Stokes profile analysis and vector magnetic fields. V - The magnetic field structure of large sunspots observed with Stokes II, ApJ, 348, $747-760$ 
Lites, B. W., Rutten, R. J., Kalkofen, W., 1993, Dynamics of the solar chromosphere. I Long-period network oscillations, ApJ, 414, 345-356

Lites, B. W., Leka, K. D., Skumanich, A., Martinez Pillet, V., Shimizu, T., 1996, SmallScale Horizontal Magnetic Fields in the Solar Photosphere, ApJ, 460, 1019

Lites, B. W., Kubo, M., Socas-Navarro, H., Berger, T., Frank, Z., Shine, R., Tarbell, T., Title, A., Ichimoto, K., Katsukawa, Y., Tsuneta, S., Suematsu, Y., Shimizu, T., Nagata, S., 2008, The Horizontal Magnetic Flux of the Quiet-Sun Internetwork as Observed with the Hinode Spectro-Polarimeter, ApJ, 672, 1237-1253

Livingston, W. C., Harvey, J., 1975, A New Component of Solar Magnetism - The Inner Network Fields, in Bulletin of the American Astronomical Society, vol. 7 of Bulletin of the American Astronomical Society, p. 346

Lockwood, M., Harrison, R. G., Woollings, T., Solanki, S. K., 2010, Are cold winters in Europe associated with low solar activity?, Environmental Research Letters, 5, 024001

Mackay, D., Yeates, A., 2012, The sun's global photospheric and coronal magnetic fields: Observations and models, Living Reviews in Solar Physics, 9

Martin, S. F., 1988, The identification and interaction of network, intranetwork, and ephemeral-region magnetic fields, Sol. Phys., 117, 243-259

Martínez González, M. J., Bellot Rubio, L. R., 2009, Emergence of Small-scale Magnetic Loops Through the Quiet Solar Atmosphere, ApJ, 700, 1391-1403, 0905.2691

Martínez González, M. J., Collados, M., Ruiz Cobo, B., 2006, On the validity of the 630 $\mathrm{nm}$ Fe I lines for magnetometry of the internetwork quiet Sun, A\&A, 456, 1159-1164

Martínez González, M. J., Collados, M., Ruiz Cobo, B., Solanki, S. K., 2007, Low-lying magnetic loops in the solar internetwork, A\&A, 469, L39-L42, 0705.1319

Martínez González, M. J., Collados, M., Ruiz Cobo, B., Beck, C., 2008, Internetwork magnetic field distribution from simultaneous $1.56 \mu \mathrm{m}$ and $630 \mathrm{~nm}$ observations, A\&A, 477, 953-965

Martínez González, M. J., Bellot Rubio, L. R., Solanki, S. K., Martínez Pillet, V., Del Toro Iniesta, J. C., Barthol, P., Schmidt, W., 2012, Resolving the Internal Magnetic Structure of the Solar Network, ApJ, 758, L40

Martínez Pillet, V., Lites, B. W., Skumanich, A., 1997, Active Region Magnetic Fields. I. Plage Fields, ApJ, 474, 810

Martínez Pillet, V., Del Toro Iniesta, J. C., Álvarez-Herrero, A., Domingo, V., Bonet, J. A., González Fernández, L., López Jiménez, A., Pastor, C., Gasent Blesa, J. L., Mellado, P., Piqueras, J., Aparicio, B., Balaguer, M., Ballesteros, E., Belenguer, T., Bellot Rubio, L. R., Berkefeld, T., Collados, M., Deutsch, W., Feller, A., Girela, F., Grauf, B., Heredero, R. L., Herranz, M., Jerónimo, J. M., Laguna, H., Meller, R., Menéndez, M., Morales, R., Orozco Suárez, D., Ramos, G., Reina, M., Ramos, J. L., Rodríguez, P., 
Sánchez, A., Uribe-Patarroyo, N., Barthol, P., Gandorfer, A., Knoelker, M., Schmidt, W., Solanki, S. K., Vargas Domínguez, S., 2011, The Imaging Magnetograph eXperiment (IMaX) for the Sunrise Balloon-Borne Solar Observatory, Sol. Phys., 268, 57-102

Mehltretter, J. P., 1974, Observations of photospheric faculae at the center of the solar disk, Sol. Phys., 38, 43-57

Metcalf, T. R., Leka, K. D., Barnes, G., Lites, B. W., Georgoulis, M. K., Pevtsov, A. A., Balasubramaniam, K. S., Gary, G. A., Jing, J., Li, J., Liu, Y., Wang, H. N., Abramenko, V., Yurchyshyn, V., Moon, Y.-J., 2006, "An Overview of Existing Algorithms for Resolving the 180degree Ambiguity in Vector Magnetic Fields: Quantitative Tests with Synthetic Data", Sol. Phys., 237, 267-296

Moll, R., Cameron, R. H., Schüssler, M., 2011, Vortices in simulations of solar surface convection, A\&A, 533, A126

Moll, R., Cameron, R. H., Schüssler, M., 2012, Vortices, shocks, and heating in the solar photosphere: effect of a magnetic field, A\&A, 541, A68

Morinaga, S., Sakurai, T., Ichimoto, K., Yokoyama, T., Shimojo, M., Katsukawa, Y., 2008, Suppression of convection around small magnetic concentrations, A\&A, 481, L29-L32

Möstl, C., Hanslmeier, A., Sobotka, M., Puschmann, K., Muthsam, H. J., 2006, Dynamics of Magnetic Bright Points in an Active Region, Sol. Phys., 237, 13-23

Muller, R., 1983, The dynamical behavior of facular points in the quiet photosphere, Sol. Phys., 85, 113-121

Muller, R., Roudier, T., 1984, Variability of the quiet photospheric network, Sol. Phys., 94, $33-47$

Muller, R., Dollfus, A., Montagne, M., Moity, J., Vigneau, J., 2000, Spatial and temporal relations between magnetic elements and bright points in the photospheric network, A\&A, 359, 373-380

Muller, R., Utz, D., Hanslmeier, A., 2011, Non-Varying Granulation and Photospheric Network During the Extended 2007 - 2009 Solar Minimum, Sol. Phys., 274, 87-97

Nagata, S., Tsuneta, S., Suematsu, Y., Ichimoto, K., Katsukawa, Y., Shimizu, T., Yokoyama, T., Tarbell, T. D., Lites, B. W., Shine, R. A., Berger, T. E., Title, A. M., Bellot Rubio, L. R., Orozco Suárez, D., 2008, Formation of Solar Magnetic Flux Tubes with Kilogauss Field Strength Induced by Convective Instability, ApJ, 677, L145-L147

Narayan, G., Scharmer, G. B., 2010, Small-scale convection signatures associated with a strong plage solar magnetic field, A\&A, 524, A3

Okamoto, T. J., Tsuneta, S., Lites, B. W., Kubo, M., Yokoyama, T., Berger, T. E., Ichimoto, K., Katsukawa, Y., Nagata, S., Shibata, K., Shimizu, T., Shine, R. A., Suematsu, Y., Tarbell, T. D., Title, A. M., 2008, Emergence of a Helical Flux Rope under an Active Region Prominence, ApJ, 673, L215-L218 
Okamoto, T. J., Tsuneta, S., Lites, B. W., Kubo, M., Yokoyama, T., Berger, T. E., Ichimoto, K., Katsukawa, Y., Nagata, S., Shibata, K., Shimizu, T., Shine, R. A., Suematsu, Y., Tarbell, T. D., Title, A. M., 2009, Prominence Formation Associated with an Emerging Helical Flux Rope, ApJ, 697, 913-922

Omara, B. J., 1976, Comments on the broadening of spectral lines by collisions with atomic hydrogen, MNRAS, 177, 551-568

Orozco Suárez, D., Bellot Rubio, L. R., del Toro Iniesta, J. C., Tsuneta, S., Lites, B. W., Ichimoto, K., Katsukawa, Y., Nagata, S., Shimizu, T., Shine, R. A., Suematsu, Y., Tarbell, T. D., Title, A. M., 2007, Quiet-Sun Internetwork Magnetic Fields from the Inversion of Hinode Measurements, ApJ, 670, L61-L64

Ortiz, A., Solanki, S. K., Domingo, V., Fligge, M., Sanahuja, B., 2002, On the intensity contrast of solar photospheric faculae and network elements, A\&A, 388, 1036-1047

Parker, E. N., 1963, Kinematical Hydromagnetic Theory and its Application to the Low Solar Photosphere., ApJ, 138, 552

Parker, E. N., 1976, Hydraulic concentration of fields in the solar photosphere. IV Evolution of fields near equipartition, ApJ, 210, 810-815

Parker, E. N., 1978, Hydraulic concentration of magnetic fields in the solar photosphere. VI - Adiabatic cooling and concentration in downdrafts, ApJ, 221, 368-377

Parker, E. N., 1955, The Formation of Sunspots from the Solar Toroidal Field., ApJ, 121, 491

Parnell, C. E., DeForest, C. E., Hagenaar, H. J., Johnston, B. A., Lamb, D. A., Welsch, B. T., 2009, A Power-Law Distribution of Solar Magnetic Fields Over More Than Five Decades in Flux, ApJ, 698, 75

Petrovay, K., Szakaly, G., 1993, The origin of intranetwork fields: a small-scale solar dynamo, A\&A, 274, 543

Pevtsov, A. A., Canfield, R. C., Metcalf, T. R., 1995, Latitudinal variation of helicity of photospheric magnetic fields, ApJ, 440, L109-L112

Pevtsov, A. A., Canfield, R. C., Sakurai, T., Hagino, M., 2008, On the Solar Cycle Variation of the Hemispheric Helicity Rule, ApJ, 677, 719-722

Pietarila, A., Pietarila Graham, J., 2013, Instrumental and Observational Artifacts in Quiet Sun Magnetic Flux Cancellation Functions, Sol. Phys., 282, 389-404

Pietarila, A., Cameron, R., Solanki, S. K., 2010, Expansion of magnetic flux concentrations: a comparison of Hinode SOT data and models, A\&A, 518, A50

Pietarila, A., Cameron, R. H., Danilovic, S., Solanki, S. K., 2011, Transport of Magnetic Flux from the Canopy to the Internetwork, ApJ, 729, 136 
Pietarila Graham, J., Danilovic, S., Schüssler, M., 2009, Turbulent Magnetic Fields in the Quiet Sun: Implications of Hinode Observations and Small-Scale Dynamo Simulations, ApJ, 693, 1728, 0812.2125

Pietarila Graham, J., Cameron, R., Schüssler, M., 2010, Turbulent Small-Scale Dynamo Action in Solar Surface Simulations, ApJ, 714, 1606-1616

Ploner, S. R. O., Schüssler, M., Solanki, S. K., Gadun, A. S., 2001, , in Advanced Solar Polarimetry - Theory, Observation, and Instrumentation, (Ed.) M. Sigwarth, vol. 236 of Astronomical Society of the Pacific Conference Series, p. 363

Pneuman, G. W., Solanki, S. K., Stenflo, J. O., 1986, Structure and merging of solar magnetic fluxtubes, A\&A, 154, 231-242

Press, W. H., Flannery, B. P., Teukolsky, S. A., 1986, Numerical recipes. The art of scientific computing, Cambridge University Press

Rabin, D., 1992, Spatially extended measurements of magnetic field strength in solar plages, ApJ, 391, 832-844

Rachkovsky, D. N., 1962, Magnetic rotation effects in spectral lines, Izvestiya Ordena Trudovogo Krasnogo Znameni Krymskoj Astrofizicheskoj Observatorii, 28, 259-270

Rees, D. E., Durrant, C. J., Murphy, G. A., 1989, Stokes profile analysis and vector magnetic fields. II - Formal numerical solutions of the Stokes transfer equations, ApJ, 339, 1093-1106

Rempel, M., Schüssler, M., 2001, Intensification of Magnetic Fields by Conversion of Potential Energy, ApJ, 552, L171-L174

Rezaei, R., Steiner, O., Wedemeyer-Böhm, S., Schlichenmaier, R., Schmidt, W., Lites, B. W., 2007, Hinode observations reveal boundary layers of magnetic elements in the solar photosphere, A\&A, 476, L33-L36

Richardson, R. S., 1941, The Nature of Solar Hydrogen Vortices, ApJ, 93, 24

Riethmüller, T. L., Solanki, S. K., Martínez Pillet, V., Hirzberger, J., Feller, A., Bonet, J. A., Bello González, N., Franz, M., Schüssler, M., Barthol, P., Berkefeld, T., del Toro Iniesta, J. C., Domingo, V., Gandorfer, A., Knölker, M., Schmidt, W., 2010, Bright Points in the Quiet Sun as Observed in the Visible and Near-UV by the Balloon-borne Observatory SUNRISE, ApJ, 723, L169-L174

Riethmüller, T. L., Solanki, S. K., van Noort, M., Tiwari, S. K., 2013, Vertical flows and mass flux balance of sunspot umbral dots, A\&A, 554, A53

Rieutord, M., Rincon, F., 2010, The sun's supergranulation, Living Reviews in Solar Physics, 7

Roddier, F., 2004, Adaptive Optics in Astronomy, Cambridge University Press 
Roudier, T., Rieutord, M., Malherbe, J. M., Vigneau, J., 1999, Determination of horizontal velocity fields at the sun's surface with high spatial and temporal resolution, A\&A, 349, 301-311

Rouppe van der Voort, L. H. M., Hansteen, V. H., Carlsson, M., Fossum, A., Marthinussen, E., van Noort, M. J., Berger, T. E., 2005, Solar magnetic elements at 0.1 arcsec resolution. II. Dynamical evolution, A\&A, 435, 327-337

Rüedi, I., Solanki, S. K., Livingston, W., Stenflo, J. O., 1992, Infrared lines as probes of solar magnetic features. III - Strong and weak magnetic fields in plages, A\&A, 263, 323-338

Ruiz Cobo, B., del Toro Iniesta, J. C., 1992, Inversion of Stokes profiles, ApJ, 398, 375-385

Rutten, R. J., Uitenbroek, H., 1991, CA II H(2v) and K(2v) cell grains, Sol. Phys., 134, $15-71$

Sánchez Almeida, J., Lites, B. W., 2000, Physical Properties of the Solar Magnetic Photosphere under the MISMA Hypothesis. II. Network and Internetwork Fields at the Disk Center, ApJ, 532, 1215-1229

Sanchez Almeida, J., Martinez Pillet, V., 1994, The inclination of network magnetic fields, ApJ, 424, 1014-1021

Sasso, C., Lagg, A., Solanki, S. K., 2011, Multicomponent He I 10830 Å profiles in an active filament, A\&A, 526, A42

Schou, J., Borrero, J. M., Norton, A. A., Tomczyk, S., Elmore, D., Card, G. L., 2012a, Polarization Calibration of the Helioseismic and Magnetic Imager (HMI) onboard the Solar Dynamics Observatory (SDO), Sol. Phys., 275, 327-355

Schou, J., Scherrer, P. H., Bush, R. I., Wachter, R., Couvidat, S., Rabello-Soares, M. C., Bogart, R. S., Hoeksema, J. T., Liu, Y., Duvall, T. L., Akin, D. J., Allard, B. A., Miles, J. W., Rairden, R., Shine, R. A., Tarbell, T. D., Title, A. M., Wolfson, C. J., Elmore, D. F., Norton, A. A., Tomczyk, S., 2012b, Design and Ground Calibration of the Helioseismic and Magnetic Imager (HMI) Instrument on the Solar Dynamics Observatory (SDO), Sol. Phys., 275, 229-259

Schrijver, C. J., Cote, J., Zwaan, C., Saar, S. H., 1989, Relations between the photospheric magnetic field and the emission from the outer atmospheres of cool stars. I - The solar CA II K line core emission, ApJ, 337, 964-976

Schröter, E. H., 1957, Zur Deutung der Rotverschiebung und der Mitte-Rand-Variation der Fraunhoferlinien bei Berücksichtigung der Temperaturschwankungen der Sonnenatmosphäre. Mit 9 Textabbildungen, ZAp, 41, 141

Schüssler, M., 1986, MHD Models of Solar Photospheric Magnetic Flux Concentrations, in Small Scale Magnetic Flux Concentrations in the Solar Photosphere, (Eds.) W. Deinzer, M. Knölker, H. H. Voigt, p. 103 
Schüssler, M., Vögler, A., 2008, Strong horizontal photospheric magnetic field in a surface dynamo simulation, A\&A, 481, L5-L8

Schwabe, M., 1844, Sonnenbeobachtungen im Jahre 1843. Von Herrn Hofrath Schwabe in Dessau, Astronomische Nachrichten, 21, 233

Seehafer, N., 1990, Electric current helicity in the solar atmosphere, Sol. Phys., 125, 219-232

Sheeley, Jr., N. R., Bohlin, J. D., Brueckner, G. E., Purcell, J. D., Scherrer, V., Tousey, R., 1975, XUV observations of coronal magnetic fields, Sol. Phys., 40, 103-121

Shimizu, T., Nagata, S., Tsuneta, S., Tarbell, T., Edwards, C., Shine, R., Hoffmann, C., Thomas, E., Sour, S., Rehse, R., Ito, O., Kashiwagi, Y., Tabata, M., Kodeki, K., Nagase, M., Matsuzaki, K., Kobayashi, K., Ichimoto, K., Suematsu, Y., 2008, Image Stabilization System for Hinode (Solar-B) Solar Optical Telescope, Sol. Phys., 249, 221-232

Shiota, D., Tsuneta, S., Shimojo, M., Sako, N., Orozco Suárez, D., Ishikawa, R., 2012, Polar Field Reversal Observations with Hinode, ApJ, 753, 157

Simon, G. W., Leighton, R. B., 1964, Velocity Fields in the Solar Atmosphere. III. LargeScale Motions, the Chromospheric Network, and Magnetic Fields., ApJ, 140, 1120

Skumanich, A., Smythe, C., Frazier, E. N., 1975, On the statistical description of inhomogeneities in the quiet solar atmosphere. I - Linear regression analysis and absolute calibration of multichannel observations of the $\mathrm{Ca} /+$ / emission network, ApJ, 200, 747-764

Socas-Navarro, H., Lites, B. W., 2004, Observational Evidence for Small-Scale Mixture of Weak and Strong Fields in the Quiet Sun, ApJ, 616, 587-593

Solanki, S. K., 1986, Velocities in solar magnetic fluxtubes, A\&A, 168, 311-329

Solanki, S. K., 1987, The Photospheric Layers of Solar Magnetic Flux Tubes, Ph.D. thesis, PhD thesis No. 8309, ETH, Zürich, (1987)

Solanki, S. K., 1989, The origin and the diagnostic capabilities of the Stokes V asymmetry observed in solar faculae and the network, A\&A, 224, 225-241

Solanki, S. K., 1993, Smallscale Solar Magnetic Fields - an Overview, Space Sci. Rev., 63, $1-188$

Solanki, S. K., 2003, Sunspots: An overview, A\&A Rev., 11, 153-286

Solanki, S. K., Brigljevic, V., 1992, Continuum brightness of solar magnetic elements, A\&A, 262, L29-L32

Solanki, S. K., Krivova, N. A., 2011, Analyzing Solar Cycles, Science, 334, 916-917

Solanki, S. K., Stenflo, J. O., 1984, Properties of solar magnetic fluxtubes as revealed by Fe I lines, A\&A, 140, 185-198 
Solanki, S. K., Keller, C., Stenflo, J. O., 1987, Properties of solar magnetic fluxtubes from only two spectral lines, A\&A, 188, 183-197

Solanki, S. K., Rueedi, I., Livingston, W., 1992a, Infrared lines as probes of solar magnetic features. V - The magnetic structure of a simple sunspot and its canopy, A\&A, 263, $339-350$

Solanki, S. K., Rueedi, I. K., Livingston, W., 1992b, , A\&A, 263, 312

Solanki, S. K., Montavon, C. A. P., Livingston, W., 1994, Infrared lines as probes of solar magnetic features. 7: On the nature of the Evershed effect in sunspots, A\&A, 283, 221-231

Solanki, S. K., Finsterle, W., Rüedi, I., 1996a, The Influence of Sunspot Canopies on Magnetic Inclination Measurements in Solar Plages, Sol. Phys., 164, 253-264

Solanki, S. K., Zufferey, D., Lin, H., Rueedi, I., Kuhn, J. R., 1996b, Infrared lines as probes of solar magnetic features. XII. Magnetic flux tubes: evidence of convective collapse?, A\&A, 310, L33-L36

Solanki, S. K., Finsterle, W., Rüedi, I., Livingston, W., 1999, Expansion of solar magnetic flux tubes large and small, A\&A, 347, L27-L30

Solanki, S. K., Usoskin, I. G., Kromer, B., Schüssler, M., Beer, J., 2004, Unusual activity of the Sun during recent decades compared to the previous 11,000 years, Nature, 431, 1084-1087

Solanki, S. K., Barthol, P., Danilovic, S., Feller, A., Gandorfer, A., Hirzberger, J., Riethmüller, T. L., Schüssler, M., Bonet, J. A., Martínez Pillet, V., del Toro Iniesta, J. C., Domingo, V., Palacios, J., Knölker, M., Bello González, N., Berkefeld, T., Franz, M., Schmidt, W., Title, A. M., 2010, SUNRISE: Instrument, Mission, Data, and First Results, ApJ, 723, L127-L133

Spruit, H. C., 1976, Pressure equilibrium and energy balance of small photospheric fluxtubes, Sol. Phys., 50, 269-295

Spruit, H. C., 1979, Convective collapse of flux tubes, Sol. Phys., 61, 363-378

Stein, R. F., Bercik, D., Nordlund, Å., 2003, Solar Surface Magneto-Convection, in Current Theoretical Models and Future High Resolution Solar Observations: Preparing for ATST, (Eds.) A. A. Pevtsov, H. Uitenbroek, vol. 286 of Astronomical Society of the Pacific Conference Series, p. 121

Steiner, O., Pneuman, G. W., Stenflo, J. O., 1986, Numerical models for solar magnetic fluxtubes, A\&A, 170, 126-137

Steiner, O., Grossmann-Doerth, U., Schüssler, M., Knölker, M., 1996, Polarized Radiation Diagnostics of Magnetohydrodynamic Models of the Solar Atmosphere, Sol. Phys., 164, 223-242 
Steiner, O., Grossmann-Doerth, U., Knoelker, M., Schuessler, M., 1998, Dynamical Interaction of Solar Magnetic Elements and Granular Convection: Results of a Numerical Simulation, ApJ, 495, 468

Stenflo, J. O., 1973, Magnetic-Field Structure of the Photospheric Network, Sol. Phys., 32, $41-63$

Stenflo, J. O., 1982, The Hanle effect and the diagnostics of turbulent magnetic fields in the solar atmosphere, Sol. Phys., 80, 209-226

Stenflo, J. O., 2010, Distribution functions for magnetic fields on the quiet Sun, A\&A, 517, A37

Stenflo, J. O., 2012, Basal magnetic flux and the local solar dynamo, A\&A, 547, A93

Stenflo, J. O., 2013, Horizontal or vertical magnetic fields on the quiet Sun. Angular distributions and their height variations, A\&A, 555, A132

Stenflo, J. O., Harvey, J. W., 1985, Dependence of the properties of magnetic fluxtubes on area factor or amount of flux, Sol. Phys., 95, 99-118

Stenflo, J. O., Keller, C. U., Gandorfer, A., 1998, Differential Hanle effect and the spatial variation of turbulent magnetic fields on the Sun, A\&A, 329, 319-328

Stix, M., 2004, The sun : an introduction, Springer

Suematsu, Y., Tsuneta, S., Ichimoto, K., Shimizu, T., Otsubo, M., Katsukawa, Y., Nakagiri, M., Noguchi, M., Tamura, T., Kato, Y., Hara, H., Kubo, M., Mikami, I., Saito, H., Matsushita, T., Kawaguchi, N., Nakaoji, T., Nagae, K., Shimada, S., Takeyama, N., Yamamuro, T., 2008, The Solar Optical Telescope of Solar-B ( Hinode): The Optical Telescope Assembly, Sol. Phys., 249, 197-220

Thornton, L. M., Parnell, C. E., 2011, Small-Scale Flux Emergence Observed Using Hinode/SOT, Sol. Phys., 269, 13

Title, A. M., Tarbell, T. D., Topka, K. P., 1987, On the relation between magnetic field structures and granulation, ApJ, 317, 892-899

Title, A. M., Tarbell, T. D., Topka, K. P., Ferguson, S. H., Shine, R. A., SOUP Team, 1989, Statistical properties of solar granulation derived from the SOUP instrument on Spacelab 2, ApJ, 336, 475-494

Tiwari, S. K., Venkatakrishnan, P., Sankarasubramanian, K., 2009, Global Twist of Sunspot Magnetic Fields Obtained from High-Resolution Vector Magnetograms, ApJ, 702, L133L137

Tiwari, S. K., van Noort, M., Lagg, A., Solanki, S. K., 2013, Structure of sunspot penumbral filaments: a remarkable uniformity of properties, A\&A, 557, A25

Topka, K. P., Tarbell, T. D., Title, A. M., 1992, Properties of the smallest solar magnetic elements. I - Facular contrast near sun center, ApJ, 396, 351-363 
Trujillo Bueno, J., Shchukina, N., Asensio Ramos, A., 2004, A substantial amount of hidden magnetic energy in the quiet Sun, Nature, 430, 326-329

Tsuneta, S., Ichimoto, K., Katsukawa, Y., Lites, B. W., Matsuzaki, K., Nagata, S., Orozco Suárez, D., Shimizu, T., Shimojo, M., Shine, R. A., Suematsu, Y., Suzuki, T. K., Tarbell, T. D., Title, A. M., 2008a, The Magnetic Landscape of the Sun's Polar Region, ApJ, $688,1374-1381$

Tsuneta, S., Ichimoto, K., Katsukawa, Y., Nagata, S., Otsubo, M., Shimizu, T., Suematsu, Y., Nakagiri, M., Noguchi, M., Tarbell, T., Title, A., Shine, R., Rosenberg, W., Hoffmann, C., Jurcevich, B., Kushner, G., Levay, M., Lites, B., Elmore, D., Matsushita, T., Kawaguchi, N., Saito, H., Mikami, I., Hill, L. D., Owens, J. K., 2008b, The Solar Optical Telescope for the Hinode Mission: An Overview, Sol. Phys., 249, 167-196

Unno, W., 1956, Line Formation of a Normal Zeeman Triplet, PASJ, 8, 108

Unno, W., 1959, Turbulent Motion in the Solar Atmosphere. I. Doppler Widths of Photospheric Lines., ApJ, 129, 375

Utz, D., Jurčák, J., Hanslmeier, A., Muller, R., Veronig, A., Kühner, O., 2013, Magnetic field strength distribution of magnetic bright points inferred from filtergrams and spectropolarimetric data, A\&A, 554, A65

van Noort, M., 2012, Spatially coupled inversion of spectro-polarimetric image data. I. Method and first results, A\&A, 548, A5

van Noort, M., Lagg, A., Tiwari, S. K., Solanki, S. K., 2013, Peripheral downflows in sunspot penumbrae, A\&A, 557, A24

Venkatakrishnan, P., 1986, Inhibition of convective collapse of solar magnetic flux tubes by radiative diffusion, Nature, 322, 156

Vitas, N., Viticchiè, B., Rutten, R. J., Vögler, A., 2009, Explanation of the activity sensitivity of Mn I $5394.7 \AA$, A\&A, 499, 301-312

Viticchié, B., Sánchez Almeida, J., 2011, Asymmetries of the Stokes V profiles observed by HINODE SOT/SP in the quiet Sun, A\&A, 530, A14

Vögler, A., Schüssler, M., 2007, A solar surface dynamo, A\&A, 465, L43-L46

Vögler, A., Shelyag, S., Schüssler, M., Cattaneo, F., Emonet, T., Linde, T., 2005, Simulations of magneto-convection in the solar photosphere. Equations, methods, and results of the MURaM code, A\&A, 429, 335-351

Wang, J., Wang, H., Tang, F., Lee, J. W., Zirin, H., 1995, Flux distribution of solar intranetwork magnetic fields, Sol. Phys., 160, 277-288

Wedemeyer-Böhm, S., Scullion, E., Steiner, O., Rouppe van der Voort, L., de La Cruz Rodriguez, J., Fedun, V., Erdélyi, R., 2012, Magnetic tornadoes as energy channels into the solar corona, Nature, 486, 505-508 
Wiegelmann, T., Solanki, S. K., Borrero, J. M., Martínez Pillet, V., del Toro Iniesta, J. C., Domingo, V., Bonet, J. A., Barthol, P., Gandorfer, A., Knölker, M., Schmidt, W., Title, A. M., 2010, Magnetic Loops in the Quiet Sun, ApJ, 723, L185-L189, 1009.4715

Wiehr, E., 1978, A Unique Magnetic Field Range for Nonspot Solar Magnetic Regions, A\&A, 69, 279

Xu, Z., Lagg, A., Solanki, S. K., 2010, Magnetic structures of an emerging flux region in the solar photosphere and chromosphere, A\&A, 520, A77

Yelles Chaouche, L., Solanki, S. K., Schüssler, M., 2009, Comparison of the thin flux tube approximation with 3D MHD simulations, A\&A, 504, 595-603

Zayer, I., Solanki, S. K., Stenflo, J. O., 1989, The internal magnetic field distribution and the diameters of solar magnetic elements, A\&A, 211, 463-475

Zayer, I., Stenflo, J. O., Keller, C. U., Solanki, S. K., 1990, Dependence of the properties of solar magnetic flux tubes on filling factor. II - Results of an inversion approach, A\&A, $239,356-366$

Zeeman, P., 1897, On the Influence of Magnetism on the Nature of the Light Emitted by a Substance., ApJ, 5, 332

Zirin, H., 1987, Weak solar fields and their connection to the solar cycle, Sol. Phys., 110, 101-107

Zirin, H., Popp, B., 1989, Observations of the 12 micron MG I lines in various solar features, ApJ, 340, 571-578 


\section{Acknowledgements}

First of all, I would like to express my thorough gratitude to Sami Solanki for his supervision and guidance throughout my $\mathrm{PhD}$ studies. His sharp and insightful advice has truly shaped my approach to all things scientific.

Special thanks must also go to all the SLAMers for creating such a warm and welcoming group. In particular Andreas Lagg deserves my thanks for his continued patience and willingness to help with any problem, both big and small, that arose during my $\mathrm{PhD}$. The crazy ping-pong matches at the VTT and your detailed introduction on how Ron Miel improves the seeing will surely remain a cherished memory. Thanks also go to Alex Feller and Michiel van Noort for their helpful explanations and detailed discussions.

A big thanks I give also to Stefan Dreizler for all his support, Dieter Schmitt for his help and the IMPRS.

Often, however, the small, mundane things are the ones that make the biggest difference and in that vein I would like to thank Shahin Jafarzadeh for being a good friend and for that unforgettable SST campaign and also Jayant Joshi for all the fun we shared in making the GM photobook. And to both of you for making our office such a great place. My deep gratitude also goes to my Mum for her unwavering support full of encouragements and care that allowed me to pursue this path and to my Dad for being an inspiration to this day. Finally, I would like to thank María Dasí Espuig for her patience and optimism, all the great trips and travels, the relentless dances and for making the biggest smallest difference every day... just thank you. 



\section{Scientific contributions}

\section{Refereed Contributions:}

Buehler, D., Lagg, A., Solanki, S.K., 2013, Quiet Sun magnetic fields observed by Hinode: Support for a local dynamo, A\&A, 555, A33

\section{Conference Contributions:}

Buehler, D., Lagg, A., Solanki, S.K., Long-term variation of quiet Sun magnetic flux patches, Hinode-4, Palermo, Italy, 11-15 October 2010, (poster)

Buehler, D., Lagg, A., Solanki, S.K., Variation of quiet Sun magnetic elements between 2006 and 2011 using Hinode SOT/SP, SDO-3 Workshop, Stanford, CA, USA, 31 October 4 November 2011, (poster)

Buehler, D., Lagg, A., Solanki, S.K., Quiet Sun magnetic fields support for a local dynamo, Hinode-6, St. Andrews, UK, 14-17 August 2012, (talk) 



\section{Lebenslauf}

\section{Persönliche Daten}

Name:

David Bühler

Geburtstag:

04.02.1987

Geburtsort:

Itzehoe

Staatsbürgerschaft:

deutsch

\section{Schulausbildung}

09/1993 - 07/1997: Grundschule Reutti

09/1997 - 12/2000: Kepler Gymnasium Ulm

01/2001 - 07/2001: Dover College

09/2001 - 07/2005: Dover Grammar School for Boys

\section{Studium}

10/2005 - 06/2009: MSci Physics with a year in Europe, Imperial College London

\section{Promotion}

02/2010 - 11/2013: Promotion an der Universität Göttingen, Stipendium des Max-Planck-Instituts für Sonnensystemforschung 